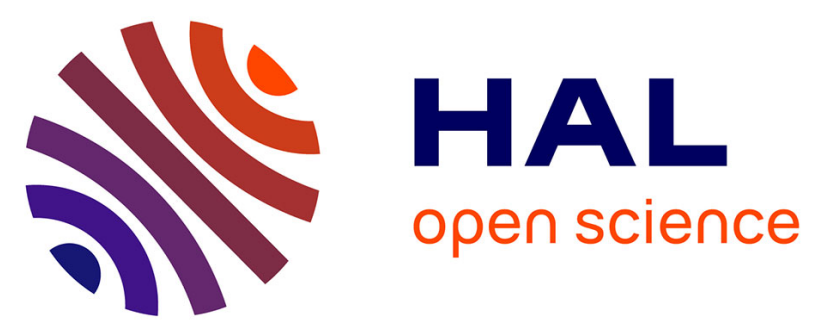

\title{
Exploiting Nonlinear Dynamics in Novel Measurement Strategies and Devices: From Theory to Experiments and Applications
}

Carlo Trigona, Salvatore Baglio, Bruno Andò, Salvatore La Malfa, Vincenzo Marletta, Adi Bulsara, Andy Kho, Visarath In, Joseph D. Neff, Gregory W. Anderson, et al.

\section{To cite this version:}

Carlo Trigona, Salvatore Baglio, Bruno Andò, Salvatore La Malfa, Vincenzo Marletta, et al.. Exploiting Nonlinear Dynamics in Novel Measurement Strategies and Devices: From Theory to Experiments and Applications. IEEE Transactions on Instrumentation and Measurement, 2011, 60 (3), pp.667-695. 10.1109/TIM.2010.2089576 . lirmm-00580562

\section{HAL Id: lirmm-00580562 https://hal-lirmm.ccsd.cnrs.fr/lirmm-00580562}

Submitted on 28 Mar 2011

HAL is a multi-disciplinary open access archive for the deposit and dissemination of scientific research documents, whether they are published or not. The documents may come from teaching and research institutions in France or abroad, or from public or private research centers.
L'archive ouverte pluridisciplinaire HAL, est destinée au dépôt et à la diffusion de documents scientifiques de niveau recherche, publiés ou non, émanant des établissements d'enseignement et de recherche français ou étrangers, des laboratoires publics ou privés. 


\title{
Exploiting Nonlinear Dynamics in Novel Measurement Strategies and Devices: From Theory to Experiments and Applications
}

\author{
Salvatore Baglio, Senior Member, IEEE, Adi R. Bulsara, Bruno Andò, Salvatore La Malfa, Vincenzo Marletta, \\ Carlo Trigona, Patrick Longhini, Andy Kho, Visarath In, Joseph D. Neff, Gregory W. Anderson, \\ Chris C. Obra, Brian K. Meadows, and Antonio Palacios
}

(Invited Paper)

\begin{abstract}
This paper is focused on the exploitation of intrinsic nonlinear dynamics toward novel measurement systems and readout methodologies. In particular, sensors that can be represented as nonlinear dynamical systems and are often reducible to systems described by a static nonlinearity are considered; the nonlinear behavior therefore reduces to the dynamics of a system characterized by two or more (meta)stable equilibrium states (or attractors) separated by energetic thresholds to be overcome to transition from one attractor to the other. The presence of a weak unknown target signal is assessed via the monitoring of the "residence times" in the attractors. This operational scenario that is based on the monitoring of suitable "events" avoids an "amplitude-based" readout and provides a very simple and sensitive readoutprocessing scheme. Many noise effects are also mitigated by the intrinsic decoupling between the amplitude domain of the input signal and the event or time domain of the output signal. We present here the general transduction methodology for this class of "residence-times difference" sensors, together with the experimental results obtained from the working versions of these sensors (in particular, a simple fluxgate magnetometer). We then introduce some novel dynamical behavior that occurs when the active nonlinear (in this case, bistable) elements are coupled using well-crafted coupling topologies. Sensors based on these coupling schemes provide several advantages over their single-element counterparts. We discuss the dynamics of the coupled-element device, summarizing recent theoretical and experimental results. Finally, we
\end{abstract}

Manuscript received October 4, 2009; revised August 24, 2010; accepted August 27, 2010. Date of current version February 9, 2011. This work was supported in part by the Office of Naval Research (Code 30) and in part by the Office of Naval Research-Global. The work of A. P. was supported in part by the National Science Foundation under Grants CMS-0625427 and CMS-0638814. The Associate Editor coordinating the review process for this paper was Dr. Reza Zoughi.

S. Baglio, B. Andò, S. La Malfa, V. Marletta, and C. Trigona are with the Dipartimento di Ingegneria Elettrica, Elettronica e dei Sistemi, Università degli Studi di Catania, 95125 Catania, Italy (e-mail: salvatore.baglio@ diees.unict.it; bruno.ando@diees.unict.it).

A. R. Bulsara, P. Longhini, A. Kho, V. In, J. D. Neff, G. W. Anderson, C. C. Obra, and B. K. Meadows are with the Space and Naval Warfare Systems Center Pacific, San Diego, CA 92152-5001 USA (e-mail: bulsara@ spawar.navy.mil).

A. Palacios is with the Nonlinear Dynamical Systems Group, Department of Mathematics and Statistics, San Diego State University, San Diego, CA $92182-$ 7720 USA.

Color versions of one or more of the figures in this paper are available online at http://ieeexplore.ieee.org.

Digital Object Identifier 10.1109/TIM.2010.2089576 describe the construction and performance of working devices (magnetic- and electric-field sensors) based on these concepts.

Index Terms-Amorphous magnetic materials, ferroelectric devices, magnetometers, nonlinear dynamical systems, sensor phenomena and characterization.

\section{NOMENCLATURE}

SNR Signal-to-noise ratio.

$R T D \quad$ Residence-times difference that is used as a quantifier of the sensor output.

RTR Residence rimes ratio.

$R T D F \quad$ Residence-times density function.

$\Delta T \quad$ Symbol used for the RTD in the equations.

$\Delta T_{R} \quad$ Symbol used for the RTR in the equations.

$R O C$ Receiver operating characteristic, which is a parameter used to quantify the attitude of a sensor to detect a target.

$H_{x} \quad$ Target magnetic field to be measured.

$H_{e} \quad$ Bias magnetic field produced by the current $I_{e}$ driven into the bias coil of a fluxgate.

$\hat{H}_{e} \quad$ Amplitude of excitation magnetic field.

$H_{c} \quad$ Coercive field of a ferromagnetic core.

$V_{o} \quad$ Output voltage.

$x \quad$ Magnetization of a fluxgate ferromagnetic core.

$U \quad$ Potential energy function used for the mean-field description of both the ferromagnetic $U(M, t)$ and the ferroelectric device $U(P, t)$.

$h(t) \quad$ Generic alternating magnetic field considered in the expression of the potential energy function and in the consequent differential equation; it includes both the bias and the target field contributions.

$S C F G$ Single-core fluxgate.

$C C F G$ Coupled-core fluxgate.

$T^{ \pm} \quad$ Time spent by the magnetization in each of the two saturation states.

$T_{\text {ob }} \quad$ "Observation time," this is the time over which the fluxgate output is cumulated and averaged to produce a useful output.

$R \quad$ Resolution.

$2 D \quad$ Noise variance. 
$\tau \quad$ Device time constant.

$\lambda \quad$ Coupling gain in the CCFG.

$\lambda_{c} \quad$ Critical value of the coupling gain, i.e., the value above which oscillations start.

$\tau_{e} \quad$ Time constant in the dynamic model of the ferroelectric device.

$\hat{E}_{e} \quad$ Amplitude of the excitation electric (E) field.

$P_{F E} \quad$ Polarization of the ferroelectric material.

$A_{F E} \quad$ Area of the ferroelectric capacitor electrode.

\section{Introduction: The "FoOtPRINT" OF This PAPER}

A LARGE class of dynamic sensors have nonlinear input-output characteristics, often corresponding to a bistable (or multistable) potential energy function that underpins the sensor dynamics. These sensors include magnetic-field sensors, e.g., simple fluxgate sensor [1], [2] and superconducting quantum interference device (SQUID) [3], [4], ferroelectric sensors [5], [6], and mechanical sensors, e.g., acoustic transducers, made with piezoelectric materials [7].

In many cases, the detection of a small dc or low-frequency target signal is based on a spectral technique[1], [2], [8] wherein a known periodic (usually sinusoidal or triangular) bias signal is applied to the sensor to switch it between its two locally stable attractors which correspond to the minima of the potential energy function, when the attractors are fixed points. In higher dimensional systems (e.g., the dc SQUID [3], [4]), bistability may correspond to a transition between an excitable steady state (characterized by a single fixed point) and an oscillatory steady state (characterized by a limit cycle). Usually, the amplitude of the bias signal is taken to be quite large, i.e., above the deterministic switching threshold which is itself dependent on the potential barrier height and the separation of the minima, in order to render the response largely independent of the noise. In this configuration, the switching events between the stable attractors are controlled by the signal.

In the presence of background noise, and the absence of the target signal, the power spectral density (PSD) of the system response contains only the odd harmonics of the bias signal frequency $\omega$. The spectral amplitude at $n \omega$ (where $n$ is even) is zero unless the asymmetrizing dc signal is present; hence, the appearance of power at $2 \omega$ and its subsequent analysis have been proposed as a detection/quantification tool for the target signal [9], given that $\omega$ is known a priori. In practice, a feedback mechanism is frequently utilized for reading out the asymmetry-producing target signal via a nulling technique [1]-[3], [8].

The aforementioned (PSD-based) readout scheme has some drawbacks. The chief among them is the requirement of large onboard power to provide the high amplitude (typically, taken to be much greater than the energy barrier separating the metastable attractors of the system) and high frequency (usually selected based on the inherent sensor properties, e.g., the various time constants associated with the dynamics) bias signal. The feedback electronics can also be cumbersome and introduce their own noise floor into the measurement, and finally, the bias signal generator often increases the noise floor in the system.
The aforementioned preamble provides an outline of readout schemes based on a computation of the PSD or an information transfer metric as an appropriate measure of the system response; this readout methodology has been widely studied, optimized, and used for the analysis of the output signal in fluxgate magnetometers [1], [2].

Alternatively, one can invoke a purely "event-based" description of the system response with an output quantifier expressed in the time domain. A similar strategy has been developed for the family of frequency output sensors [10], [11]. In this paper, we focus not on the frequency output but on a measurement technique that is based on the evaluation of system "residence times" in its steady states [12], [13]. For a two-state system, the residence time in one of the stable steady states is defined as the time elapsed between the crossing of one threshold (a crossing "event") and the crossing of the opposite threshold (the following "event").

In the presence of a noise background, the residence times in the stable states have random components. The residence time statistics in a bistable system were proposed for the first time in [14] as a quantifier for the stochastic resonance (SR) [15] phenomenon which involves subthreshold driving signals, so that the threshold crossing events are, because of necessity, noise assisted. They have also been studied in a prototype bistable model system [16], [17]. The important features of residencetimes distributions are often seen in neurophysiological experimental data; it is widely believed that the "point process" generated by successive neural "firing" events contains much relevant information about the stimulus that leads to the firing [18]-[20]. Under the appropriate conditions on the spike train, most importantly a "renewal" characterization, corresponding to uncorrelated threshold crossing events, [21] it is possible to connect the "interspike interval histogram" (the RTDF, in the language of this paper) to the output PSD [22], [23].

The RTD-based readout has some advantages compared to the conventional (PSD-based) readout scheme: It can be implemented without the knowledge of the (computationally demanding) PSD of the system output, which is the most critical component needed being an adequate counter. We hasten to note that threshold statistics underpin the class of "levelcrossing detectors" that have been available for a variety of applications for almost 50 years [24]. The method outlined before has, in different forms, been used in nonlinear sensors (particularly sensors that have a hysteretic output-input transfer characteristic), albeit without much effort paid in considering the effects of sensor noise on the measurement process [8].

In this paper, we will describe the use of threshold crossing statistics [24] to gain information regarding the presence of small unknown target signals in a nonlinear dynamic detector, taken to be a two-state system (i.e., underpinned by a bistable potential energy function). We will see that the specific (E- and magnetic-field) sensors considered in this paper are, in fact, underpinned by dynamics that fall under the two-state paradigm.

Recently [25], [26], we have demonstrated that coupling an odd number $N \geq 3$ of overdamped bistable elements in a ring, with unidirectional coupling, and ensuring that at least one of them has an initial state that is different from the others 
can lead to oscillatory behavior when the coupling strength exceeds a critical value. The characteristics of the bifurcation to oscillatory behavior depend on the system dynamics and, more importantly, the manner in which the elements are coupled. For the case of Duffing dynamics with additive interelement coupling [25], the system undergoes a Hopf-like bifurcation to oscillatory behavior; the oscillation frequency is nonzero infinitesimally past the bifurcation point and increases as one goes deeper into the bifurcation regime. In [25] and [26], we considered a system of coupled elements having "soft-potential" dynamics, characteristic (for example) of hysteretic single-domain ferromagnetic cores. This work has led to exploiting the emergent oscillatory behavior for signal detection purposes: Specifically, an external symmetry-breaking dc target signal having small amplitude (usually, much smaller than the energy barrier height of a single element) can be detected and quantified via its effect on the oscillation frequency and (temporal) asymmetry of the oscillation waveforms.

We reiterate that the previously outlined behavior (the analogy to a frustrated spin system comes to mind) occurs only for an odd number of elements $N \geq 3$ with cyclic unidirectional coupling and initial conditions selected so that at least one state point is different from the others. For even $N$, the oscillatory behavior occurs only when $N$ exceeds a critical value; we do not consider this situation in this paper.

We start with a prototypical system, a single ferromagnetic element (which would be the active material or core in a single-core magnetometer) which we treat as an overdamped dynamical system whose response to a very weak (compared to the energy barrier height) target magnetic field is quantified via the RTD readout strategy involving a sinusoidal or triangular suprathreshold bias signal. Along the way, we describe the RTD and its ramifications, including the effects of a noise floor, and a phenomenological "SNR" that depends on the noise floor, and the observation time. We then describe how this measurement strategy is implemented in an SCFG magnetometer, providing a detailed description of the actual experimental device and the dependence of its performance on various parameters that enter into the characterization and measurement process. The SCFG magnetometer is placed in context with existing magnetometers that do the same task, but with a conventional (i.e., based on the response PSD) readout.

Following the treatment of the single-core magnetometer, we continue to sensors that are underpinned by coupled active elements. We provide an overview of the dynamics, stemming from our choice of coupling scheme, and describe how a small external perturbation consisting of a dc target signal can be detected via its effects on the aforementioned emergent oscillatory behavior in the system response. We describe how the response (of single- and coupled-core sensors) can be quantified in the presence of a noise floor by considering the device "resolution" as a (noise-dependent) figure of merit. We also describe qualitatively the effects of increasing the number of active elements, as well as the effects of the noise floor on the system response. Our treatment is extended to a coupled-element E-field sensor (EFS) with each active element consisting of a ferroelectric capacitor.

Wherever necessary, we introduce new physical and mathematical ideas and tools in the specific context of the system under consideration. It will become clear that one can use a reductionist approach to describe the qualitative features of the dynamics of a class of nonlinear sensors (for $N=1$, as well as the coupled case $N \geq 3$ ); however, there are important differences that set the individual sensor types (e.g., magnetic and E fields) apart, e.g., the structure and functional form of the (bistable) potential energy function that underpins the specific kind of system under consideration. Put succinctly, the reductionist approach serves a useful purpose insofar as it allows us to study the qualitative features of the response that are endemic to a rather wide class of systems; however, it does have its limitations, and these are revealed when one takes into account the (important) differences in the dynamics of the individual elements that comprise separately the magnetic-field sensor and EFS.

\section{SCFG MAGNETOMETER}

Fluxgate magnetometers, which are one of the sensors considered in this paper, have always been of interest to the technical and scientific communities as practical and convenient sensors for magnetic-field measurements requiring a resolution around $1 \mu \mathrm{T}$ at room temperature; they have found applicability [2], [27]-[29] in fields such as space and geophysical exploration and mapping, and nondestructive testing, as well as assorted military applications. Recently, the possibilities offered by new technologies and materials in realizing miniaturized devices with improved performance have led to renewed interest in a new generation of cheap, compact, and low-power fluxgate sensors. However, their miniaturization is complicated by the rapid increase of magnetic noise with the reduction of the device dimensions and the general practical requirements for achieving high sensitivity (large number of windings, large cross-sectional sensor area, and large bias current) which, however, are at odds with the desired characteristics (low cost, power, and noise) of the miniaturized sensors. Nonetheless, despite the difficulties manifested in integrated devices with better performance, the literature does contain good examples of fluxgate sensors in printed circuit boards (PCBs) [30], [31] and even CMOS [32]. In particular, CMOS affords the possibility of realizing the sensing part (fluxgate) and the readout circuit on the same chip, resulting in enhanced reliability and lower costs in batch production.

Today's highly specialized fluxgate devices boast laboratory noise floors as low as $10 \mathrm{pT} / \sqrt{\mathrm{Hz}}$ and are used in a variety of magnetic remote sensing applications [33]-[38]. However, one must take these performance quantifiers with the caveat that, when operated unshielded in a practical application, the detection of target signals above the noise floor is limited by the ambient magnetic field (which, in the case of the terrestrial magnetic field, can have nonstationary, as well as random, components). Hence, various techniques often involving a reference magnetometer for the purposes of subtraction of the noise floor from the output of the target-sensing device must be employed [39] if one wishes to take advantage of the low noise floor.

One of the earliest fluxgate designs was due to Forster [2]; it consists of two detection coils connected in a differential arrangement and two excitation coils, as shown in Fig. 1. Two 
(a)

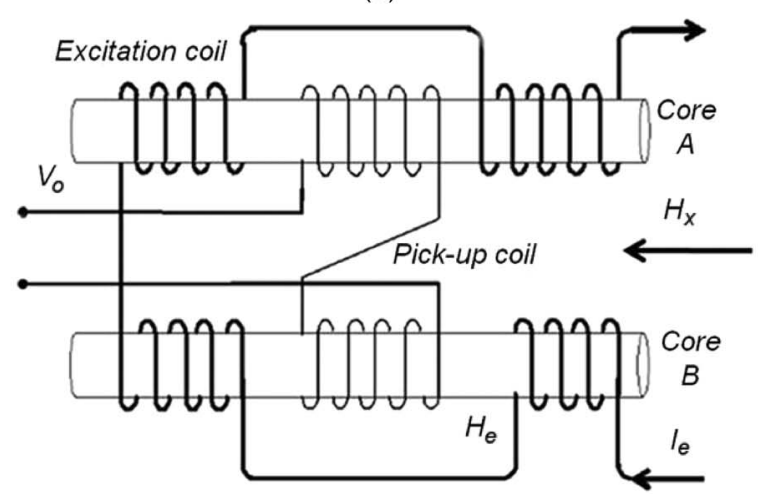

(b)

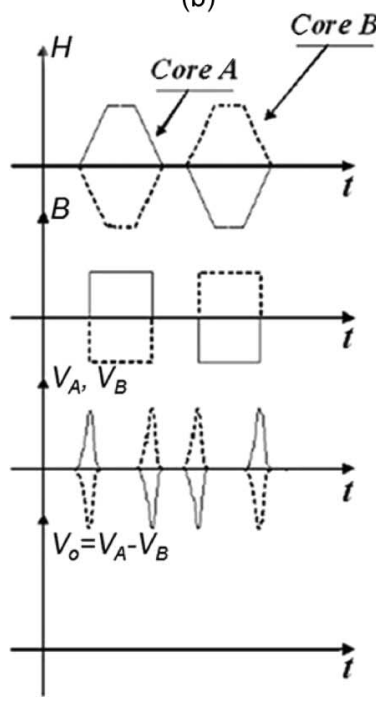

(c)

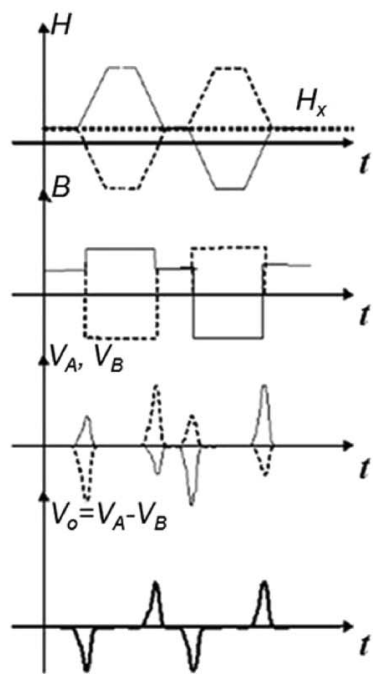

Fig. 1. (a) Arrangement for a traditional (single-core) fluxgate magnetometer. (b) Qualitative time evolution of signals with zero external target magnetic field (the output is zero). (c) In the presence of a nonzero target magnetic field. In particular, the magnetizing field $H$, the corresponding magnetic flux $B$, the voltages induced at the pickup coils $V$, and the resulting output voltage $V_{o}(t)$ are shown in the (b) absence and (c) presence of the target magnetic field $H_{x}$ (figure reproduced with permission from [40]).

periodic counterphased magnetic fluxes are induced through the excitation currents $I_{e}$, driving the magnetic cores periodically into saturation in opposite senses: The pulsed signals $V_{i}(t)$, where $i=A, B$, given by each pickup coil, oscillate back and forth at the forcing frequency. The time waveforms of these signals are shown in Fig. 1(b) and (c), respectively. For a symmetric core magnetization and in the absence of the target signal, the output voltage signal, denoted by $V_{o}(t)=V_{A}(t)-$ $V_{B}(t)$, is zero. An external (assumed to be dc or extremely low frequency) magnetic field $H_{x}$ leads to an asymmetry in the core magnetization and hence produces a nonzero output voltage $V_{o}(t)$ [bottom panel in Fig. 1(c)]. Today's instruments are usually operated in a "locked" mode wherein a feedback mechanism is utilized to readout the asymmetry produced by the target signal via a nulling technique.

Among recent advances in fluxgate sensor technology, the so-called "fluxset" devices [41] (see also [28] for good overviews), which differ from a conventional fluxgate in the way they convert the magnetic field into an output electrical signal, are noteworthy. The fluxset magnetometer is based on

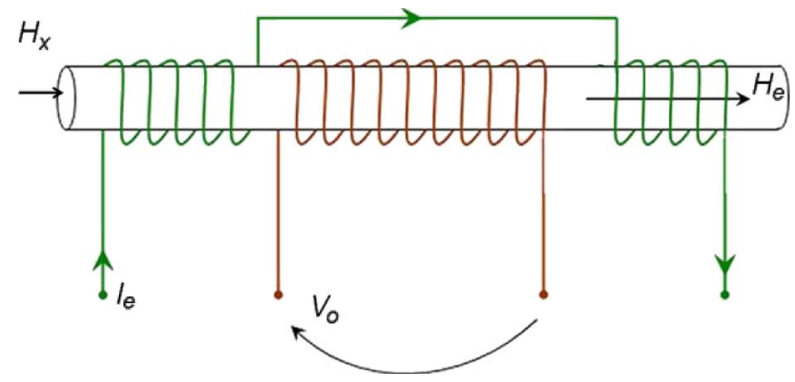

Fig. 2. Schematic representation of the RT-fluxgate device.

the influence of the external magnetic field on the time necessary to produce the reversal magnetization of the ferromagnetic core under a periodic magnetic excitation. Given an optimal core design and an excitation field, the time shift depends only on the value of the external (target) magnetic field. Hence, the measurement of the magnetic-field amplitude via a voltage measurement in the fluxgate is replaced by a high-accuracy time measurement that can be rendered more impervious to clutter signals and noise via an understanding of the device response in the presence of a noise floor. Such a (deterministic) "time-domain" description was first introduced by Strycker and Wulkan [42]. The simplest implementation of our "residencetimes" fluxgate (RT-fluxgate) is shown in Fig. 2. It is based on a two-coil structure (a primary coil and a secondary coil) wound around a suitable magnetic core. The magnetization of the core is governed by the excitation field $H_{e}$ produced in the primary coil, and the core is composed of a ferromagnetic material with the characteristic "sharp" input-output hysteresis loop, corresponding to a bistable potential energy function, which underpins the system dynamics; the minima of this potential energy function correspond to the two (stable) steady magnetization states. In order to reverse the core magnetization, a suprathreshold excitation field is required. Here, the "threshold" represents the minimum field required to switch the saturation of the material; mathematically, it corresponds to the inflection point(s) in the potential, it being tacitly assumed that, once this point is crossed, the state point will relax to the stable attractor on that side, on a timescale (dependent on the time constant $\tau$ ) far smaller than the other time constants (the signal period and the inverse noise bandwidth) that govern the bias signal and noise dynamics.

With an alternating excitation (or bias) magnetic field $H_{e}$, the output voltage $V_{o}$ at the secondary coil will be alternating and symmetric in time. The presence of an external "target" magnetic field $H_{x}$ (taken to be dc throughout this paper) will break this symmetry, and the resulting temporal asymmetry can be used to monitor the target field amplitude. In the following section, we elucidate the RTD approach via a simple model.

Before proceeding further, it is instructive to schematize the relationship between the representative dynamics of an overdamped system (in this case, a ferromagnetic sample characterized by an average magnetization variable) underpinned by a potential energy function and the familiar hysteresis behavior of the ferromagnetic sample: This relationship is shown in Fig. 3(a) and (b), while Fig. 3(c) shows the effect, on the 

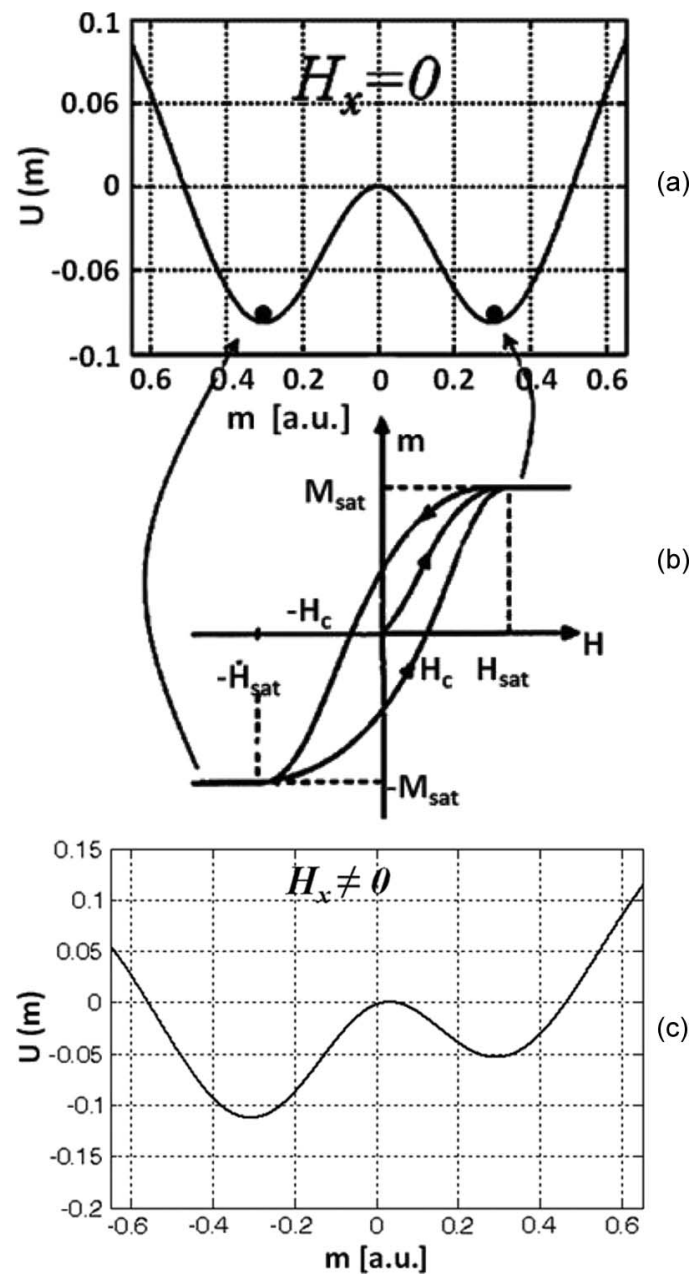

Fig. 3. Qualitative depiction of the dynamics of a typical overdamped bistable system (in this case, a ferromagnetic sample described by the mean-field theory) with state point (the "average" magnetization variable $M$ ) evolving as $\tau(d M / d t)=-\nabla U(M)$. (a) Potential energy function $U(M)$. (b) Hysteresis characteristic. The (stable) potential minima correspond to the saturation states $\pm M_{\text {sat }}$ of the hysteresis cycle; the locations of these stable minima correspond to the points $\pm H_{\text {sat. }}$. $H_{c}$ is the coercivity of the sample; it corresponds roughly to the deterministic switching threshold and can be calculated in terms of the potential energy parameters (see text). (c) When a dc magnetic field $H_{x}$ is present, the effect on the potential function, at a given time instant, is additional skewing which reflects itself in the (unequal) mean residence times in the two stable steady states. Dimensionless units are implied on all axes (figure reproduced with permission from [43]).

potential energy function, of an external (dc) applied magnetic field.

We now turn our attention to the (single-core) fluxgate magnetometer, with the goal of arriving at a device that exploits the intrinsic nonlinear dynamics of the active material (the ferromagnetic core).

\section{A. Deterministic Mean-Field Dynamics in a Ferromagnetic Sample: The Working Principle of the SCFG}

One of the best known systems that exhibits hysteresis [44] is a ferromagnetic material, usually described by the Ising-type models [44], [45] and exhibiting a phase transition to the paramagnetic state when the temperature $T$ exceeds the Curie temperature $T_{c}$. One may describe the ferromagnet by a Ginzburg-Landau free-energy function that incorporates the relevant order parameter (in this case, the core magnetization); this potential energy function is then bistable in the ferromagnetic phase, becoming monostable in the paramagnetic phase. The transition to monostability can also be achieved by applying an external magnetic field which breaks the symmetry of the potential, causing one of the metastable states to disappear when the field amplitude exceeds a critical value; the system returns to bistability as soon as this field is turned off. The aforementioned considerations beg the question of having a continuum model in which one may incorporate the dynamical behavior of the ferromagnet, including the effects of time-dependent external magnetic fields. This is accomplished through the mean-field theory [45] which allows one to use a master equation for the averaged magnetization $x(t)$ and arrive at the dynamic equation

$$
\tau \frac{d x}{d t}=-x+\tanh \left[\frac{x+h(t)}{T}\right] \equiv-\nabla_{x} U(x, t)
$$

where $\tau$ is a system time constant and $T$ is a dimensionless temperature. $h(t)$ is an external magnetic field that may be time dependent, having the dimension of $x(t)$. We have also expressed (1) in terms of the gradient of a potential energy function (the analog of the free-energy function mentioned earlier)

$$
U(x, t)=\frac{x^{2}}{2}-\frac{1}{c} \ln \cosh [c\{x+h(t)\}]
$$

where we set $c=T^{-1}$. Note that, in these units, the Curie temperature is $T_{c}=z J / k_{B}$, where $z$ is the number of the nearest neighbors, $J$ is the strength of the exchange interaction, and $k_{B}$ is Boltzmann's constant. The potential energy function (2) is bistable for $c>1$; the typical behavior of this system has already been depicted (in the absence of the time-dependent bias signal $h(t)$ ) in Fig. 3. A dynamical hysteresis in the system (1) and other systems with qualitatively similar potential energy functions, with $h(t)$ often taken to be time sinusoidal, has been the subject of much recent study [46]-[49]; cooperative phenomena, e.g., SR, arising in the presence of background fluctuations [48]-[50] have also been examined in the literature. The role of background fluctuations has been ignored in the derivation of (1); however, later in this paper, we will discuss the effects of noise on the device performance.

For the purpose of making contact with experimental results, we are interested in a "macroscopic" magnetic description of the fluxgate dynamics rather than a detailed micromagnetic description based on individual domain dynamics; a detailed derivation of mean-field dynamics of the form (1) is not our intent. Rather, we use an equation of the form (1) to model the dynamics of the entire core, assuming the applicability of the mean-field description. Such modeling has been used in the literature [1], [8], and we find that the model yields good (given that it is, at best, an approximation to a detailed micromagnetic description of the domain dynamics) agreement with the experimental results, thereby validating our description. Specifically, we assume that our ferromagnetic sample is only one magnetic domain thick, this being the rigorous limit of the applicability 
of the dynamics (1). Other collective approaches to the stochastic dynamics of aggregates of monodomain ferromagnetic particles do exist in the literature [51], usually starting from the Landau-Gilbert equations [52] for a single-domain element with thermal noise included; SR in such a system has also been studied [53].

In practice, the time constant $\tau$ is very important, particularly in the presence of noise. If $\tau$ is the smallest timescale in the system, i.e., both the noise bandwidth (defined, for Gaussian noise, as the inverse of the correlation time $\tau_{c}$ ) and the bias signal period are well within the system bandwidth $\tau^{-1}$, then the device behaves like a static nonlinearity. Hence, the dynamics are reduced to following the dynamics of the noise plus the signal, as they traverse two static thresholds, given essentially by the fixed points of the potential (2); in the presence of a timeperiodic bias signal, these "deterministic switching thresholds" are functions of the signal amplitude and frequency [54]. It is convenient to start our description [13], [55] of the deterministic dynamics with this assumption and a suprathreshold bias signal $H_{e}(t)$ which can be sinusoidal or triangular. We note that, in practical devices, the bias signal is known and controllable; hence, we will assume always that its parameters can be varied at will. The bias signal plays an important role. In most practical detection scenarios, the target magnetic field (assumed dc) is too small to engineer transitions between the stable steady states of the potential energy function (2); hence, one applies the known suprathreshold time-periodic bias signal to force the dynamics to switch between the stable steady states (on a timescale that depends on the amplitude and frequency of the bias signal). In the presence of the target dc signal $H_{x}$, the potential energy function is a priori skewed with one energy well being shallower than the other, as noted earlier [Fig. 3(c)]. This asymmetry manifests itself in the (now unequal) residence times in the two steady states. In conventional (frequencydomain-based) readout schemes, the output PSD contains only odd harmonics of the bias frequency $\omega$ when the target signal is absent; this is easily apparent when one realizes that the dynamics (1) contain only odd terms in the state variable in this case. As already outlined, the presence of a nonzero target signal leads to the appearance of even harmonics of the frequency $\omega$ in the output PSD so that the occurrence of power in the $2 \omega$ bin is an indication of the presence of the target signal, and the spectral amplitude at $2 \omega$ yields a measure of the strength $H_{x}$ of the target signal. Hence, conventional fluxgate magnetometers that use this quantifier of the target magnetic field are often called "second-harmonic fluxgates" [1], [2], [8], [27], [28]. We note, in passing, that the forcing term $h(t)+H_{x}$ (with the definition $h(t)=\hat{H}_{e} \sin \omega t$ ) is inside the nonlinearity in (1), which is a direct consequence of the mean-field nature of the magnetic interaction [45].

We now obtain simple expressions for the RTD $\Delta T=$ $\left|T^{+}-T^{-}\right|$, with $T^{ \pm}$denoting the residence times in the two stable steady states. Consider first the simplest possible manifestation of a two-state system, which is the Schmitt trigger (ST) [56], characterized by a two-state output and a hysteretic transfer characteristic. Its output rests in one state as long as the input is less than a threshold. The switch to the other state is almost instantaneous (the ST can be considered the limiting

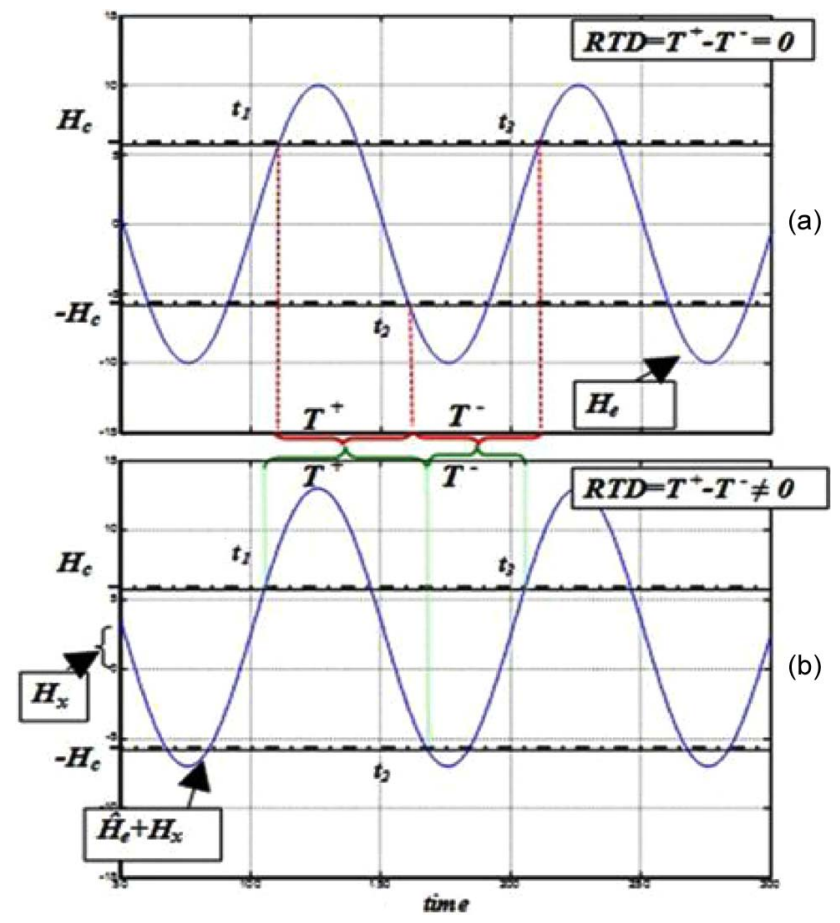

Fig. 4. RTD readout scheme schematized for a sinusoidal bias signal $h(t)$ and (a) target signals $H_{x}=0$ and (b) $H_{x}>0$ (figure reproduced with permission from [40]).

case of a dynamical system [57] with very small time constant $\tau$ ), occurring when the input exceeds the threshold.

In the device considered here, let $\pm H_{c}$ be the upper and lower thresholds, with $h(t)$ being the suprathreshold time-sinusoidal bias signal having period $T_{0} . H_{x}\left(\ll H_{c}\right)$ is a dc target signal whose effect is to "shift" the sinusoidal signal upward by an amount $H_{x}$.

In Fig. 4, the qualitative behavior of the signals involved is shown in order to help demonstrate the working principle for the RTD fluxgate, also denoted by us as the SCFG magnetometer.

Crossings of the upper and lower thresholds occur at times $t_{1}$ and $t_{2}$ computed via

$$
\begin{aligned}
H_{x}+\hat{H}_{e} \sin \left(\omega t_{1}\right) & =H_{c} \\
H_{x}-\hat{H}_{e} \sin \left[\omega\left(t_{2}-\frac{T_{0}}{2}\right)\right] & =-H_{c}
\end{aligned}
$$

so that the residence times are given by $T^{+}=t_{2}-t_{1}$ and $T^{-}=t_{3}-t_{2}$. This yields for the RTD (the subscript $s$ denotes the sinusoidal bias signal)

$$
\Delta T_{s}=\frac{2}{\omega}\left[\arcsin \left(\frac{H_{c}+H_{x}}{\hat{H}_{e}}\right)-\arcsin \left(\frac{H_{c}-H_{x}}{\hat{H}_{e}}\right)\right] .
$$

One may define a "sensitivity" via $S_{s}=d \Delta T_{s} / d H_{x}$ (this is readily seen to have the form of the slope of an input-output transfer function)

$$
\frac{\omega \hat{H}_{e}}{2} S_{s}=\left(1-\left(\frac{H_{c}+H_{x}}{\hat{H}_{e}}\right)^{2}\right)^{-1 / 2}+\left(1-\left(\frac{H_{c}-H_{x}}{\hat{H}_{e}}\right)^{2}\right)^{-1 / 2}
$$


which clearly increases with $H_{x}$, saturating at $\bar{H}_{x}=\hat{H}_{e}-$ $H_{c}$. Note that (as expected) $\Delta T_{s}$ vanishes when $H_{x}=0$ and $\Delta T_{s} \rightarrow\left(4 H_{x} / \omega \hat{H}_{e}\right)$ for large (compared to the threshold location) $\hat{H}_{e}$, so that the sensitivity is rendered independent of the target signal in this limit. In the large $\hat{H}_{e}$ regime, we can also show that the residence time $T^{+} \rightarrow(1 / \omega)\left(\pi+\left(2 H_{x} / \hat{H}_{e}\right)\right)$, which approaches $T_{0} / 2$ at very large $\hat{H}_{e}$, as expected. A completely analogous set of limiting values exists for the other residence time $T^{-}$

As evident in Fig. 4, the sensitivity depends on the slope of the total signal $H_{x}+\hat{H}_{e} \sin (\omega t)$ at its intersection with the threshold value $H_{c}$, which is the coercive field of the magnetic core. Referring to the signal slope at the intersection if, as assumed here, the external (target) signal $H_{x}$ is constant or, at least, very slow with respect to the bias signal $\hat{H}_{e} \sin \omega t$, the slope at the intersection will depend only on the bias signal. Therefore, in the context of the system sensitivity, one observes that a fixed change in $H_{x}$, i.e., a reduction of the bias signal slope at the crossing point, corresponds to an increase in the RTD and, therefore, to an increase in the device sensitivity. However, the sensitivity $S_{s}$ depends on the (instantaneous) value of the target signal $H_{x}$, which is not always desirable in a practical device. Note however that, for $H_{x} \ll \hat{H}_{e}$, one may expand the right-hand side of (4) and (5) to first order in $H_{x} / \hat{H}_{e}$, which leads to a sensitivity that is independent of $H_{x}$. Another option is to use a triangular bias signal given by

$$
\begin{aligned}
& h_{t}(t)=\alpha t-\frac{T_{0}}{4}<t<\frac{T_{0}}{4}+n T_{0} \\
& h_{t}(t)=-\alpha\left(t-\frac{T_{0}}{2}\right) n T_{0}+\frac{T_{0}}{4}<t<\frac{3}{4} T_{0}+n T_{0}
\end{aligned}
$$

with $n(>0)$ being a positive integer. The slope $\alpha$ is conveniently given by $\alpha=d h_{t}(t) / d t=4 \hat{H}_{t} / T_{0}$, with $\hat{H}_{t}$ being the maximum amplitude of the (triangular) bias signal. As before, one writes down the crossing times via the relations

$$
H_{x}+\alpha t_{1}=H_{c} \quad H_{x}-\alpha\left(t_{1}-\frac{T_{0}}{2}\right)=-H_{c}
$$

which lead, as before, to the RTD (the subscript $t$ denotes the triangular bias waveform)

$$
\Delta T_{t}=\frac{4 H_{x}}{\alpha}
$$

so that the sensitivity is given by

$$
S_{t}=\frac{4}{\alpha}=\frac{2 \pi}{\omega \hat{H}_{t}}
$$

with $\hat{H}_{t}>H_{x}+H_{c}$. This sensitivity is always independent of the target signal $H_{x}$, and this is usually a desirable device feature. It is important to note that too large a value of the target signal $H_{x}$ will effectively render the potential energy function monostable, thereby eliminating switching events completely; of course, such large target signals can be easily detected by far simpler techniques. In order for switching events to occur reliably, one can readily see that the constraint $\left|H_{x}\right|<$ $\left(\hat{H}_{t}-H_{c}\right)$ must be fulfilled; for the sinusoidal bias signal, the same condition applies, with $\hat{H}_{t}$ replaced by the sinusoidal bias amplitude $\hat{H}_{e}$.

To complete the aforementioned discussion, it is necessary to provide a definition (in terms of the parameters of an actual device) of the critical magnetic field $H_{c}$ and to make contact with the actual device dynamics, as represented by (1) and (2). In practice, $H_{c}$ represents the coercivity of the core; it can be thought of as a (deterministic) switching threshold and represents the minimum value of the applied field that would permit a switch to the opposite potential energy well. Clearly, in order for this to happen, the saddle point and one of the potential minima must coalesce into a point of inflection, at the value of $H_{c}$. To compute the coercivity, we assume that $h(t)=H_{c}$ and note that the potential (2) has inflection points at $x_{f s p}=\sqrt{c-1 / c}=-x_{f s m}$. We can readily show that the positive and negative values of the coercivity (depending on which branch of the hysteresis loop is under consideration) are given by

$$
\begin{array}{ll}
x_{f s p}=\sqrt{\frac{c-1}{c}} & H_{c}=\frac{1}{c} \tanh ^{-1} x_{f s p}-x_{f s p} \\
x_{f s m}=-\sqrt{\frac{c-1}{c}} & H_{c}=-\frac{1}{c} \tanh ^{-1} x_{f s p}+x_{f s p}
\end{array}
$$

whence the RTD $\Delta T_{s}$ can be readily calculated via (4).

It is important to reiterate that the preceding treatment rests on the assumption of the magnetometer as a near-static nonlinearity. In the presence of a suprathreshold bias signal that effectively sets the "clock" for the transitions between the steady states, and whose frequency is well within the sensor bandwidth $\tau^{-1}$, one would expect the aforementioned expressions for the RTD and sensitivity to provide a good description of the temporal behavior of the device. The important point is that, in the RTD approach, "events" (corresponding to threshold crossings) are considered rather than "signal amplitudes" (as necessary in a conventional PSD-based readout). This approach leads to an extremely simple readout circuitry, mainly based on a fast clock and counterkeeping a running arithmetic mean (necessary when a noise floor is present) of the RTD.

It is instructive to carry out a more formal comparison between the two readout techniques. We address this point now, using the deterministic framework developed so far. The computation of the RTD and associated sensitivity in the presence of a noise floor is deferred to a later section and will, as we shall see, depend on the relative magnitudes of certain important timescales in the dynamics.

\section{B. Comparing the RTD Readout to Traditional (PSD-Based) Readout Techniques}

The working principle of the Forster magnetometer [2] has been described by Primdahl [58] and is shown in Fig. 1(a). Two cylindrical cores A and B are exposed to the target dc field $H_{x}$ and the excitation (or bias) field $H_{e}(t)=\hat{H}_{e} \sin \omega t$ induced in opposite phases through an exciting current $I_{e}$ flowing in the coils. If the core material has a hysteretic $B-H$ loop, the working point $P$ describes the magnetic state of both cores, with $B$ being the induction in each of them and the net 
induction through a common secondary coil being zero, since the fluxes are opposed in the two cores. When the dc target field $H_{x}$ is applied, it splits the working point $P$ into two points $B_{A}$ and $B_{B}$ representing cores $\mathrm{A}$ and $\mathrm{B}$, respectively, on the $B-H$ curve. For very small $H_{x}$, we can linearize around $H_{e}$, obtaining a constant permeability $(\mu)$ value, with the induction through the common secondary being

$$
\Delta B=B_{A}-B_{B} \approx 2 \frac{H_{x}}{1+D \mu} \frac{d B}{d H}=2 \frac{H_{x}}{1+D \mu} \mu
$$

where $B$ is generically considered as a function of $H(t) . D$ is the demagnetizing factor [44], [58]. It arises from the tendency of the applied bias field to introduce magnetic poles at the ends of the cores, and these poles distort the field; effectively, the magnetic poles tend to lower the coil field. The output voltage is calculated from (11)

$$
\begin{aligned}
V_{o} & =-N_{s} A_{s} \frac{d \Delta B}{d t}=-2 N_{s} A_{s} \frac{(1+D \mu)-D \mu}{(1+D \mu)^{2}} \frac{d \mu}{d t} \\
& =-2 \frac{N_{s} A_{s} H_{x}}{(1+D \mu)^{2}} \frac{d^{2} B}{d H^{2}} \frac{d H}{d t}
\end{aligned}
$$

where $N_{s}$ denotes the number of turns of the secondary coil and $A_{s}$ is the cross-sectional area. If we now consider a sinusoidal biasing current $I_{e}(t)=\hat{I}_{e} \sin \omega t$ that induces a biasing magnetic field expressed as $H_{e}(t)=\hat{H}_{e} \sin \omega t$, an analytical expression for the induced output voltage can be computed once the analytical expression for $B(H)$ is available. Here, $\hat{I}_{e}$ and $\hat{H}_{e}$ represent the bias current amplitude and the corresponding magnetic-field amplitude, respectively. The procedure for doing so has been described in [58] and results in the following expression for the amplitude at the second harmonic of the bias frequency:

$$
V_{o}=\omega N_{s} A_{s} H_{x}\left(3 a_{2} \hat{H}_{e}^{2}+5 a_{5} \hat{H}_{e}^{4}+\ldots\right) \sin 2 \omega t .
$$

The second-harmonic component is used as a good quantifier of the target dc signal since, in the absence of the target signal, the response would contain only the odd harmonics of $\omega$, as is well known for a symmetric transfer characteristic. The sensitivity, characterized via the appearance of the second harmonic in the response, is computed as

$$
S_{2}=\frac{\partial V_{o}}{\partial H_{x}}=\omega N_{s} A_{s}\left(3 a_{2} \hat{H}_{e}^{2}+5 a_{5} \hat{H}_{e}^{4}+\cdots\right) .
$$

It is evident that the conditions that lead to the optimal response in the RTD readout strategy are not applicable in the aforementioned case where sensitivity is proportional to both the bias signal frequency and (the powers of) the amplitude. In fact, for an integrated version of the magnetometer, it can be observed that the very parameters (e.g., the frequency $\omega$ and the dimension parameters $N_{s}$ and $A_{s}$ ) that, on the basis of (14), could be adjusted to enhance the sensitivity in the second-harmonic readout scheme could degrade the sensor performance when the RTD readout strategy is adopted; here, we recall (see Section II-A) that the sensitivity in the case of the RTD readout strategy does not depend on the geometrical parameters of the core. Finally, it is seen that the requirements

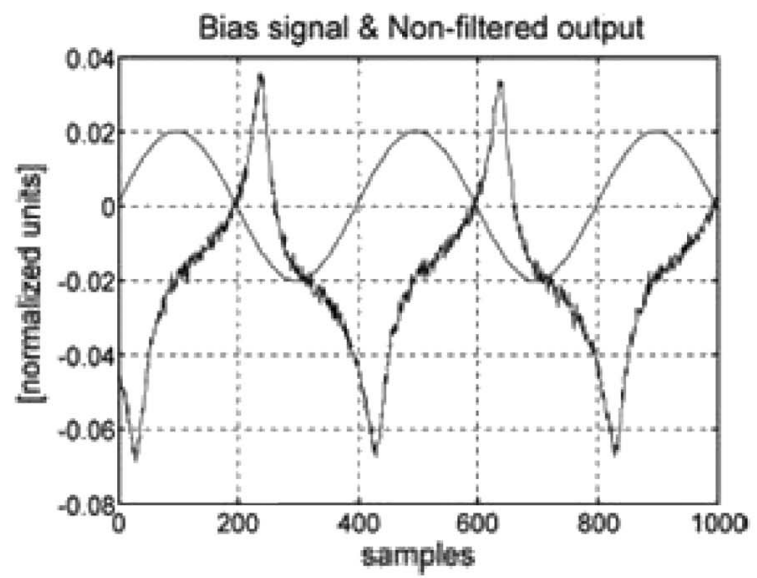

(a)

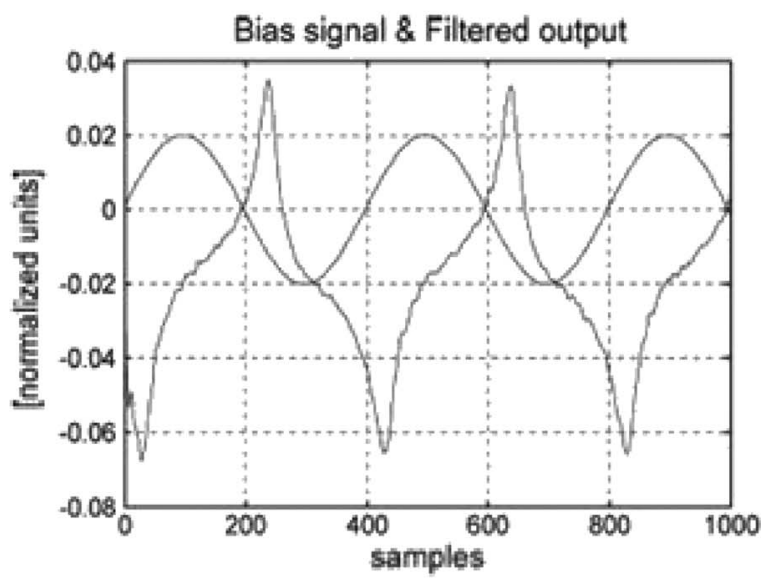

(b)

Fig. 5. (a) Raw output waveform corrupted by noise. The sinusoidal curve is the (sinusoidal in this case) bias signal $H_{e}(t)$. (b) Filtered output signal after postprocessing (figure reproduced with permission from [40]).

for improving sensitivity in the RTD approach allow for simultaneously reducing the power budget of the device; this latter feature is particularly appealing for practical realizations of the device.

\section{RTD Readout in the Presence of a Noise Floor}

A noise floor is usually present in the magnetometer and forces one to adjust the RTD readout to take note of its presence. In the experimental magnetometers (which will be described later in this paper), considerable trouble is taken to lower the noise floor, e.g., employing ultralow-noise components in the readout circuitry, using low-noise cores, etc. Despite these precautions, there is a noise floor which is clearly visible, in Fig. 5, for example. This figure shows a typical acquired (voltage) waveform from the experimental setup. The noise does not introduce a significant degradation (at least in the experiments that produced this waveform) in the readout, since the latter is no longer based on the output signal level but on the position of the peak value. In the presence of noise, the procedure for estimating the residence times from the output voltage peaks involves repetitive observations of the output and is based on averaging and resampling postprocessing; the filtered signal is shown in the bottom panel, and the noise (which is filtered from 


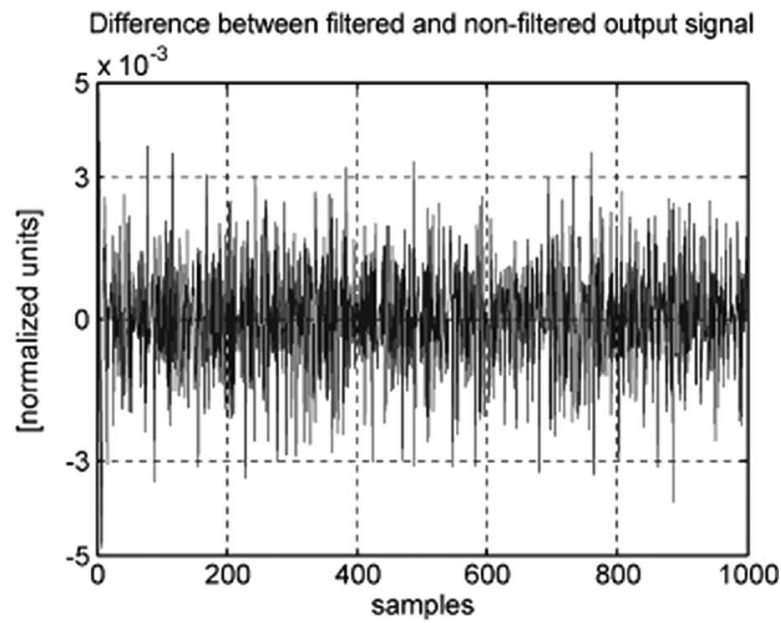

(a)

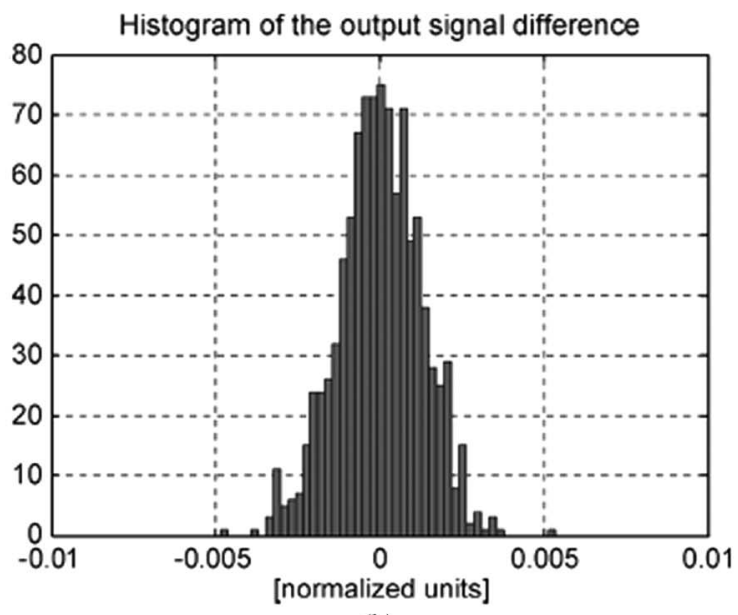

(b)

Fig. 6. (a) Residual between filtered and unfiltered output signals. (b) Histogram of the residual between the raw output signal and the filtered data (figure reproduced with permission from [40]).

the output waveform) is seen to have a symmetric distribution (Fig. 6) which is reminiscent of a Gaussian but only becomes Gaussian in the singular limit of extremely low noise variance.

Based on the aforementioned (and other similar) results of quantifying the noise in the output waveform, we assume the noise to be Gaussian and exponentially correlated, i.e., it is derived from a white-noise-driven Ornstein-Uhlenbeck process [59]

$$
\dot{\zeta}(t)=-\tau_{c}^{-1} \zeta+F(t)
$$

where $F(t)$ is a "white" noise process having zero mean and variance $2 D:\langle F(t)\rangle=0$, and $\left\langle F(t) F\left(t^{\prime}\right)\right\rangle=2 D \delta\left(t-t^{\prime}\right)$. The correlation function of the colored Gaussian noise is $\left\langle\zeta(t) \zeta\left(t^{\prime}\right)\right\rangle=\left\langle\zeta^{2}\right\rangle \exp \left[-\left|t-t^{\prime}\right| / \tau_{c}\right]$, where $\left\langle\zeta^{2}\right\rangle=D \tau_{c}$. In the limit of vanishing correlation time $\left(\tau_{c} \rightarrow 0\right)$, the band-limited Gaussian noise $\zeta(t)$ becomes delta correlated or "white" [59]. We also assume that the bias signal frequency $\omega$ is well within the noise band, i.e., the noise $\zeta(t)$ is wideband vis-a-vis the signal. It will become evident that it may be possible to somewhat mitigate problems arising from the noise statistics by adaptively increasing the bias signal amplitude in real scenarios.
For $\hat{H}_{e}$ suprathreshold, the threshold crossings to the stable states are controlled by the signal, but the noise does introduce some randomness into the interspike intervals. The result is a broadening of the (left- and right-well) RTDFs. For $\hat{H}_{e}$ far above the deterministic switching threshold and moderateto-low noise $\left(\sqrt{2 D} \ll \hat{H}_{e}\right)$, the RTDF assumes a symmetric narrow shape with a mean value (the mean residence time) that is nearly the same as the most probable value or mode (this is the value around which most experimental observations are likely to be clustered). As the signal amplitude decreases and/or the noise intensity increases, the RTDF starts to develop a tail, so that the mean and mode get separated; the appearance of the tail is an indication of the increased role of noise in producing switching events, although the suprathreshold signal is still the dominant mechanism. When the signal amplitude falls below the deterministic crossing threshold, the crossings are driven largely by the noise. The RTDF can assume a characteristic multipeaked structure [14], [16], [19] that shows a "skipping" behavior since the noise can actually cause the crossings to occur at different multiples $n T_{0} / 2$ (where $n$ is odd), where $T_{0}$ is the bias signal period, and the SR scenario can come into play [60] through a synchronization of characteristic timescales in the system; the noise determines the tail of the RTDF and introduces a broadening or dispersion in individual lobes of the RTDF, since the individual crossing events do not always occur precisely at times $n T_{0} / 2$. We will not consider this subthreshold case in this paper since, as already noted, the bias signal is always taken to be suprathreshold due to the "soft" core material.

It is important to note that, with zero target signal, the crossing statistics to the left or right minimum of the potential are identical with coincident RTDFs, as should be expected. However, throughout this paper, we focus on a nonzero but small dc target signal $H_{x} x_{0} \ll U_{0}$ ( $U_{0}$ being the energy barrier height and $\pm x_{0}$ denoting the locations of the minima of the potential energy function, in the absence of noise and bias signal). We will also consider the presence of a noise floor and of the suprathreshold bias signal $h(t)=\hat{H}_{e} \sin \omega t$ that was already introduced in the preceding section. The following observations can then be made.

1) The potential (2) is now a priori skewed even for $\hat{H}_{e}=$ 0 . Hence, the mean residence times in the two stable states will be different (the energy barrier that has to be overcome in making a switch is different, depending on the direction of the switch). We denote these times by the ensemble-averaged quantities $\left\langle T^{ \pm}\right\rangle$, respectively.

2) For very large bias signal amplitudes and moderate noise intensity, the RTDFs are two well-separated symmetric near-Gaussian distributions centered about modes that coincide with their means; for signal amplitudes much larger than the rms noise amplitude, the distributions tend to coincide.

3) The separation $\langle\Delta T\rangle=\left|\left\langle T^{+}\right\rangle-\left\langle T^{-}\right\rangle\right|$of the mean values yields a direct measure of the asymmetry-producing target signal. It can be calculated for the zero noise case, as well as with weak noise and bias signal amplitude $A$ that is well suprathreshold. We find, in fact, that, in 
the large $\hat{H}_{e} / \sqrt{2 D}$ limit, q is well approximated by its deterministic analog and is proportional to the asymmetrizing signal $H_{x}$.

4) In the presence of increasing amounts of noise, the RTDs broaden, tending to merge, and their mean values (which are now well separated from the modes) also may be difficult to distinguish since $\langle\Delta T\rangle \rightarrow 0$ with increasing noise. However, increasing the bias signal amplitude (this could be done adaptively in a real application) once again leads to the signal as the dominant mechanism for crossing events, and the distributions "sharpen" somewhat and have less overlap, becoming more resolvable, even though the separation $\langle\Delta T\rangle$ may actually decrease.

5) For very special situations, primarily those in which there is a small amount of noise, one can carry out the aforementioned procedure with a very weak bias signal. In this case, the RTDs for each potential well are almost unimodal with long tails. The mean values and modes are again dependent on the target signal; however, in this case, the slopes of the long-time tails of the density functions are different for the two wells, and this difference can also be used as an identifier, if needed, of the target signal. The limiting case of zero bias signal has also been studied [12]; our studies indicate that this operating mode may be optimal even for small target signals $H_{x}$, with $\langle\Delta T\rangle$ proportional to $H_{x}$. This operating mode relies on the presence of background noise that is strong enough to initiate interwell switching events without the presence of a suprathreshold bias signal. Of course, in practical applications, the presence of assorted (often non-Gaussian and nonstationary) noise sources, as well as readout issues, could make the zero bias signal mode a possibility for only very specialized scenarios. For these more complicated noise backgrounds, the renewal assumption for the crossing events cannot be expected to hold. This operation mode may be particularly well suited for applications wherein the potential barrier height can be adjusted during an experiment. It does afford the attractive possibility of significantly reduced onboard power.

6) Our calculations to date indicate that a sinusoidal bias signal is not always optimal; in some operational scenarios, better sensitivity may be obtained by using other signal waveforms, e.g., the triangular waveform already discussed earlier, and using this waveform leads to "local linearity" at the crossing points of the bias signal and the thresholds. Another waveform obtained through a combination of square and triangular signals is described in [13]; it can be adjusted to have a stepwise linear behavior at the intersection with the threshold.

Note that, in an experiment, under any of the aforementioned scenarios, it is not necessary to actually accumulate successive RTDs. One simply maintains a running arithmetic mean of the residence times. Then, an important issue is the amount of data (dependent on the response time of the electronics), the amount of time (the observation time $T_{\mathrm{ob}}$ to be addressed hereinafter) one can "look" at the target signal, as well as the bias frequency $\omega$ required to obtain reliable estimates of $\langle\Delta T\rangle$. It is clear that increasing the bias signal amplitude, in order to better discriminate the RTDs, can lead to enhanced detection probabilities. In this context, it is important to point out that the aforementioned technique may be implemented with bias signal amplitudes that are not substantively larger than the potential barrier height and also with relatively low bias frequencies. In practice, however, one should expect to confront a tradeoff between the bias signal amplitude (this is a function of the onboard power in a practical sensor) and the concomitant degree of resolution of the peaks of the histograms, and what is necessary for a reliable estimate, usually with a limited observation time, of the target signal from $\langle\Delta T\rangle$; as already noted, for a slightly suprathreshold bias signal, the background noise degrades the measurement, and this can be readily seen at the level of the RTD, which, for a slightly suprathreshold bias signal, has a distinctive noise-dependent tail which shrinks as the bias signal is raised further above the threshold.

The aforementioned observations are well backed by numerical simulations [13], [61]; in the interests of space conservation, we do not show these results here. Instead, after a semirigorous discussion of performance measures, we will describe some experimental results with laboratory devices.

\section{Theoretical Performance Measure}

Following the results of the preceding section, one may ask the logical question: What is the optimal detector configuration for a given target signal in a noise background? In general, of course, the optimal detector would be a linear (energy) detector unless (as in the current case) the sensor is inherently nonlinear. As discussed in earlier theoretical work [12], one can obtain (theoretically, at least) a measurable $\langle\Delta T\rangle$ for zero bias signal, provided that the noise is sufficiently large to cause the sensor to switch between its stable steady states without the need for the externally applied bias signal; this scenario would also have the added benefits of lower onboard power and a lower noise floor since a substantial part of the noise floor arises from the bias signal generator. In real-life applications, of course, one is usually constrained by a finite observation time $T_{\mathrm{ob}}$, so that in a sensor which has been painstakingly constructed to have the lowest possible noise floor, the noise-induced switching events might be too few and far between to take advantage of the noise-activated nonlinear dynamic sensor [12] scenario. Put differently, the noise intensity must be high enough to yield an acceptable (for the purpose of computing a reliable measure of the response) switching rate; else, the controlled bias signal becomes necessary.

When considering the optimal bias signal amplitude, one must note that increasing the bias signal amplitude reduces $\Delta T$ even as it renders the RTDs somewhat more resolvable for large noise. This indicates that, in a practical application, it may not necessarily be of benefit to apply an extremely large bias signal; as already mentioned before, bias signals having amplitude not much larger than the barrier height will suffice. Our simulations show that, past a certain value, increasing the bias signal amplitude has no effect on the detection probability [13], [61]. This point has been discussed in some detail in recent articles by 
Dari et al. [62], [63], wherein the response of the nonlinear sensor is characterized via its ROCs, which are plots of detection versus false-alarm probabilities as a function of the detection threshold for different operating conditions. These authors compute and plot the area under the ROC as a function of the bias signal amplitude. For a "perfect" sensor, this area would be unity, corresponding to a unit detection probability and a zero false-alarm probability; in practice, there is usually a nonzero false-alarm probability, with a concomitant reduction of the detection probability, that lowers (below unity) the area under the ROC. The ROC-based analysis shows that the detection probability decreases rapidly when the bias signal amplitude decreases below the deterministic switching threshold. Hence, using a bias signal that is at or beyond the deterministic switching threshold will improve the sensor detection performance with the added caveat that this enhanced performance will require a concomitant increase in the onboard power budget. This tradeoff (between an acceptable false-alarm rate and onboard power) is thus an important point in the design and operation of practical sensors; it also has implications in the sensor noise floor, with a larger amplitude bias signal (usually) bringing along an increase in the noise-floor stemming directly from the signal generator. Other exceptional cases, e.g., large noise or non-Gaussian and/or nonstationary noise, may also necessitate the application of larger bias signals. An ROC analysis for the RTD magnetometer described in this paper has recently been carried out [64]. Finally, we note a recent work [65] wherein a neural-network-based processor is used to construct the input signal from the output of a nonlinear sensor. The technique involves the construction of an "error model" which incorporates the error sources in the measurement chain, as well as the propagation of the errors through the chain.

We now introduce a quantifier that closely resembles a response SNR, computed in terms of $\langle\Delta T\rangle$ and $T_{\mathrm{ob}}$. We discuss this SNR in light of our earlier (numerically inspired) observations on the behavior of the RTDF. We start by assuming that we have collected $N$ samples for each of the residence times $T_{n}^{ \pm}$. The mean values of the two RTDs are $\left\langle T_{n}^{ \pm}\right\rangle$; as discussed earlier, these may be computed directly from the crossing-time data sets (the subscript $n$ denotes an experimental or simulated quantity). The actual mean values $\left\langle T^{ \pm}\right\rangle$are then given by

$$
\left\langle T^{ \pm}\right\rangle=\left\langle T_{n}^{ \pm}\right\rangle \quad\left\langle\delta T_{n}^{ \pm}\right\rangle=\frac{\sigma_{T_{n} \pm}}{\sqrt{N}}
$$

where $\sigma_{T_{n}^{ \pm}}$is the standard deviation of each distribution and $\left\langle\delta T_{n}^{ \pm}\right\rangle$represents the uncertainty inherent in the measurement process. Then, the mean difference in residence times may be written in terms of the experimentally obtained quantities

$$
\langle\Delta T\rangle=\left\langle\Delta T_{n}\right\rangle
$$

where $\left\langle\Delta T_{n}\right\rangle=\left\langle T_{n}^{+}\right\rangle-\left\langle T_{n}^{-}\right\rangle$, and the uncertainty $\delta\left\langle\Delta T_{n}\right\rangle$ can be obtained from (16) as

$\delta\left\langle\Delta T_{n}\right\rangle=\sqrt{\delta T_{n}^{+2}+\delta T_{n}^{-2}}=\sqrt{\frac{\sigma_{T_{n}^{+2}}+\sigma_{T_{n}^{-2}}}{N}} \approx \sigma_{T_{n}} \sqrt{2 / N}$ where we set $\sigma_{T_{n}^{+}} \approx \sigma_{T_{n}^{-}}=\sigma_{T_{n}}$, since the intervals $T_{n}^{ \pm}$are assumed to be uncorrelated, with their distributions being identical and the separation of means being the only manifestation of the presence of the target signal.

The aforementioned treatment leaves us with an experimental observable (the mean RTD $\left\langle\Delta T_{n}\right\rangle$ ), together with the (noise-induced) uncertainty $\delta\left\langle\Delta T_{n}\right\rangle$ in its measurement. It is convenient to combine these quantities into a dimensionless ratio that serves the role of a response "SNR"

$$
S N R=\frac{\left\langle\Delta T_{n}\right\rangle}{\delta\left\langle\Delta T_{n}\right\rangle}=\frac{\left\langle\Delta T_{n}\right\rangle}{\sigma_{T_{n}}} \sqrt{\frac{N}{2}} .
$$

We assume that we are given a finite observation time $T_{\mathrm{ob}}=$ $2 N\left(T^{+}+T^{-} / 2\right)$, whence we can obtain

$$
N=\frac{T_{\mathrm{ob}}}{T^{+}+T^{-}}=\frac{T_{\mathrm{ob}}}{\left\langle\Delta T_{n}\right\rangle+2\left\langle T^{-}\right\rangle} \approx \frac{T_{\mathrm{ob}}}{2\left\langle T_{-}\right\rangle} .
$$

Hence, we finally obtain for the SNR (note that it is a function of all the system parameters and, specifically, of the bias signal amplitude $A$ )

$$
S N R=\frac{1}{2} \frac{\left\langle\Delta T_{n}\right\rangle}{\sigma_{T_{n}}} \sqrt{\frac{T_{\mathrm{ob}}}{\left\langle T_{n}^{-}\right\rangle}} .
$$

Note that the inverse quantity $S N R^{-1}$ in (21) is occasionally referred to as the relative standard uncertainty [66].

It is of interest to (theoretically) compute and analyze the SNR (21) as a function of the bias amplitude $A$ and other system parameters as a means to optimize performance. The simple threshold description given in Section II affords us an analytic computation of the SNR, which we now describe. It is important to reiterate, at this point, the stringent constraints on our use of the threshold representations of the magnetometer dynamics. The noise standard deviation must be small compared to the threshold "height," with $A$ being suprathreshold; the latter also affords the replacement of the dynamics (1) by the simple static threshold description that leads to the deterministic results (4) and (8). Finally, the overriding constraint of the sensor time constant $\tau$, being the smallest timescale in the dynamics, must be satisfied. To get an analytical estimate of the SNR (21), we can then resort to our simple ST model described in Section II. We assume the noise floor to be small (compared to the threshold setting or height) and to manifest itself in a fluctuating threshold with mean value $H_{c}$ (see Fig. 4); the small (compared to all other timescales in the problem) sensor time constant $\tau$ ensures that, in fact, this is the case (this is also seen in experiments). The threshold $(\psi)$ fluctuations are assumed to be Gaussian

$$
P(\psi)=\frac{1}{\sqrt{2 \pi \sigma^{2}}} \exp \left\{-\frac{\left(\psi-H_{c}\right)^{2}}{2 \sigma^{2}}\right\} .
$$

Fig. 6 shows that the Gaussian stationarity assumption is not a bad one. In (22), the variance parameter (associated with the threshold fluctuations) would be roughly the same as the equaltime correlation function $\left\langle\zeta^{2}\right\rangle$ of the (Gaussian colored) noise parameter $\zeta(t)$ in (15).

Let us consider the case of sinusoidal bias signal. Assuming that we start at $t=0$, the first $t_{1}$ to the upper threshold (at $+H_{c}$ ) 
is now a random variable; its probability density function may be readily computed [24] via a change of variables, wherein the mean crossing time is well approximated by the deterministic crossing time, as derived in Section II

$\left.P\left(t_{1}\right)=\frac{\omega \hat{H}_{e}}{\sqrt{2 \pi \sigma^{2}}} \cos \omega t_{1} \exp \left\{-\frac{\hat{H}_{e}^{2}}{2 \sigma^{2}}\left(\sin \omega t_{1}-\sin \omega t_{10}\right)^{2}\right)\right\}$

which is normalized to unity over the interval $0 \leq t_{1} \leq T_{0} / 4$, which contains the first crossing to the upper threshold. Note that $P\left(t_{1}\right)=0$ outside this interval. In an analogous manner, we obtain the first crossing-time probability for the lower threshold

$\left.P\left(t_{2}\right)=\frac{\omega \hat{H}_{e}}{\sqrt{2 \pi \sigma^{2}}} \cos \omega t_{2} \exp \left\{-\frac{\hat{H}_{e}^{2}}{2 \sigma^{2}}\left(\sin \omega t_{2}-\sin \omega t_{20}\right)^{2}\right)\right\}$

normalized to unity in $T_{0} / 2 \leq t \leq 3 T_{0} / 4$. Note that these density functions tacitly assume a deterministic threshold crossing picture of the form described in Section II. The bias signal must be well suprathreshold, and the noise intensity $\sigma^{2}$ also should be small compared to the threshold height. In (23) and (24), the deterministic crossing times $t_{1,20}$ are obtained by solving the (3) for $t_{1,2}$.

In terms of the density functions (23) and (24), we may write down formal expressions for the mean crossing times $\left\langle t_{1}\right\rangle_{t h}$ and $\left\langle t_{2}\right\rangle_{t h}$, the subscript denoting the theoretical (in this case, approximate) quantity

$$
\begin{aligned}
\left\langle t_{1}\right\rangle_{t h} & =\int_{0}^{T_{0} / 4} P\left(t_{1}\right) t_{1} d t_{1} \\
\left\langle t_{2}\right\rangle_{t h} & =\int_{T_{0} / 2}^{3 T_{0} / 4} P\left(t_{2}\right) t_{2} d t_{2} .
\end{aligned}
$$

The theoretical difference in residence times is then

$$
\begin{aligned}
\langle\Delta T\rangle_{\mathrm{th}} & =\left\langle T^{+}\right\rangle_{\mathrm{th}}-\left\langle T^{-}\right\rangle_{\mathrm{th}} \\
& =2\left(\left\langle t_{2}\right\rangle_{\mathrm{th}}-\left\langle t_{1}\right\rangle_{\mathrm{th}}\right)-T_{0}
\end{aligned}
$$

in terms of the definitions (25) and (26). The standard deviation in the denominator of (21) is computed via the second moment of $t_{1}$

$$
\sigma_{T_{n}} \approx \sqrt{2\left(\left\langle t_{1}^{2}\right\rangle_{\mathrm{th}}-\left\langle t_{1}\right\rangle_{\mathrm{th}}^{2}\right)} \equiv \sqrt{2 \sigma_{t_{1}}^{2}}
$$

and the remaining term in the denominator of the square root factor in (21) is replaced by the difference in the mean crossing times.

The aforementioned integrals must be computed numerically, in general. We then readily observe that, in the limit of small noise variance and large bias amplitude, the averaged quantities are well approximated by their deterministic counterparts

$$
\left\langle t_{1,2}\right\rangle_{\mathrm{th}} \approx t_{1,20} \quad\langle\Delta T\rangle \approx \Delta T
$$

where the deterministic RTD is given in (4) and (8) for the sinusoidal bias signal and the triangular one, respectively. We may also, in the regime of validity of the correspondences (29), approximately evaluate [13] the integrals (25) and (26) using a second-order Laplace expansion [67], in which we retain terms up to $O\left(\sigma^{2}\right)$ only. We then obtain

$$
\begin{aligned}
& \left\langle t_{1}\right\rangle_{\mathrm{th}}=t_{10}+\frac{\sigma^{2}}{\hat{H}_{e}^{2}} \sec \omega t_{10} G_{10}\left(t_{10}\right)+\text { h.o.t. } \\
& \left\langle t_{2}\right\rangle_{\mathrm{th}}=t_{20}+\frac{\sigma^{2}}{\hat{H}_{e}^{2}} \sec \omega t_{20} G_{20}\left(t_{20}\right)+\text { h.o.t. }
\end{aligned}
$$

For the variance $\sigma_{t_{1}}^{2}$, we obtain

$$
\sigma_{t_{1}}^{2} \approx \frac{\sigma^{2}}{\hat{H}_{e}^{2}} \sec \omega t_{10}\left\{G_{2}\left(t_{10}\right)-2 t_{10} G_{10}\left(t_{10}\right)\right\}
$$

where the functions $G$ have been computed in [13] and are not rewritten here; they are not essential to our description of the behavior of the RTD in the strong bias signal (and/or weak noise) regime.

The mean crossing times (30) agree very well (in the limit of small $\sigma / \hat{H}_{e}$ ) with the values obtained by numerically evaluating the integrals (25) and (26). Good agreement is also obtained between the standard deviation $\sigma_{t_{1}}$ and its numerically obtained counterpart. In fact, a glance at the equations (30) shows that, at large signal amplitude (and/or small noise intensity), the crossing times approach their deterministic values $t_{1,20}$; in turn, these behave as $1 / \hat{H}_{e}$ for large $\hat{H}_{e}$. In this regime of operation, the crossing-time density functions (23) and (24) collapse into Gaussians, having the form

$$
P\left(t_{1}\right) \approx \frac{1}{\sqrt{2 \pi \Sigma^{2}}} \exp \left\{-\frac{1}{2 \Sigma^{2}}\left(t_{1}-t_{10}\right)^{2}\right\}
$$

which is normalized to unity on $[-\infty, \infty]$ and where $\Sigma^{2}=$ $\sigma^{2} / \hat{H}_{e}^{2} \omega^{2}$, a "dressed" variance that is seen to decrease rapidly with decreasing $\sigma$ and/or increasing $\hat{H}_{e}$. A corresponding expression is obtained for $P\left(t_{2}\right)$. Note that simple differentiation of the densities (23) and (24) shows the modes approaching the mean values in the large $\hat{H}_{e} / \sigma$ limit. Of course, we have already observed (30) that the average crossing times approach their deterministic counterparts in this limit.

In the Gaussian limit, we can find a theoretical expression for the SNR. We start by computing the RTDF for the upstate (i.e., the right hand or positive well of the potential energy function) for which individual residence times are denoted by $T^{+}=t_{2}-$ $t_{1}$, with $t_{1,2}$ being the individual crossing times. The density function of the residence times is obtained via the convolution

$$
P\left(T^{+}\right)=\int_{-\infty}^{\infty} P_{1}\left(T^{+}-t_{2}\right) P_{2}\left(t_{2}\right) d t_{2}
$$

which, after some manipulations, yields

$$
P\left(T^{+}\right)=\frac{1}{\sqrt{4 \pi \Sigma^{2}}} \exp \left\{-\frac{1}{4 \Sigma^{2}}\left(T^{+}-t_{10}+t_{20}\right)^{2}\right\} .
$$


An analogous expression may be computed for the RTDF in the downstate. Then, using (4), setting $\sigma_{T_{n}}^{2}=2 \Sigma^{2}$, and taking $\left\langle T^{+}\right\rangle \approx t_{20}-t_{10}$, we obtain the theoretical SNR as

$$
S N R=\frac{1}{2} \frac{\hat{H}_{e} \omega}{\sigma_{T_{n}}} \frac{\Delta T}{\sqrt{T_{0}-\Delta T}} \sqrt{T_{\mathrm{ob}}}
$$

We note the $\hat{H}_{e} / \sigma$ dependence of the (theoretical) SNR for the sinusoidal bias signal considered here; this implies improved performance with increasing $\hat{H}_{e}$ and/or a lowered noise standard deviation. The former must be, however, balanced against the (possible) need for low onboard power in a practical system, as well as the increased noise floor contributed by the (sinusoidal) waveform generator. With increasing $\hat{H}_{e},\langle\Delta T\rangle$ decreases, and the lobes of the RTD converge to a single sharp peak at $T_{0} / 2$; in essence, a very large (and suprathreshold) $\hat{H}_{e}$ linearizes the device. The SNR increases as $\sqrt{T_{\mathrm{ob}}}$; increasing the observation time leads to better statistics (and averaging). However, in practical applications, the observation time could be limited. At this point, the observation time should be carefully defined. $T_{\mathrm{ob}}$ is the interval during which the temporal averaging window (that one "slides" over the data) is updated. Finally, we recall that the target signal $H_{x}$ enters via the deterministic crossing times $t_{1,20}$.

The aforementioned SNR computation was achieved without a (direct) calculation of the RTDF. If necessary though, this density can be calculated via the convolution of the individual densities $P\left(T^{ \pm}\right)$, and it can be evaluated in the large $\hat{H}_{e} / \sigma$ limit via the convolution and a Laplace expansion of the integrals [67]. The result is [13], [55]

$$
P(\Delta T)=\frac{1}{\sqrt{8 \pi \Sigma^{2}}} \exp \left[-\frac{\left(\Delta T-\Delta T_{d}\right)^{2}}{8 \Sigma^{2}}\right]
$$

with the subscript $d$ denoting the deterministic value.

We note in passing that, in light of the result shown in Fig. 5, the Gaussian (in the limit considered in this section) nature of the density function $P(\Delta T)$ should not occasion any surprise.

To conclude this section, we introduce a (noise-dependent) performance measure, called the resolution. This quantity is the minimum magnetic-field change that can be discriminated by the sensor against the background, after ambient static (homogeneous) magnetic fields have been nulled out, as is done when the sensor is deployed in the mode of detecting small changes in the ambient magnetic field. The resolution is defined as [66]

$$
R=S T D(\Delta T) /\left[\partial\langle\Delta T\rangle / \partial H_{x}\right]
$$

where $\langle\Delta T\rangle$ represents the averaged RTD, $S T D(\Delta T)$ is the standard deviation of $\Delta T$, and the denominator is simply the slope of the output-input transfer characteristic (the plot of $\langle\Delta T\rangle$ versus the target signal $H_{x}$ ) and represents the device responsivity. For small target signals, we expect this slope to be independent of $H_{x}$ (i.e., $\langle\Delta T\rangle \propto H_{x}$ ); this is, of course, convenient for practical applications and has been discussed earlier. The numerator in (37) is the standard deviation of the RTD. The resolution is seen to be roughly the inverse of an SNR. In laboratory characterizations involving actual

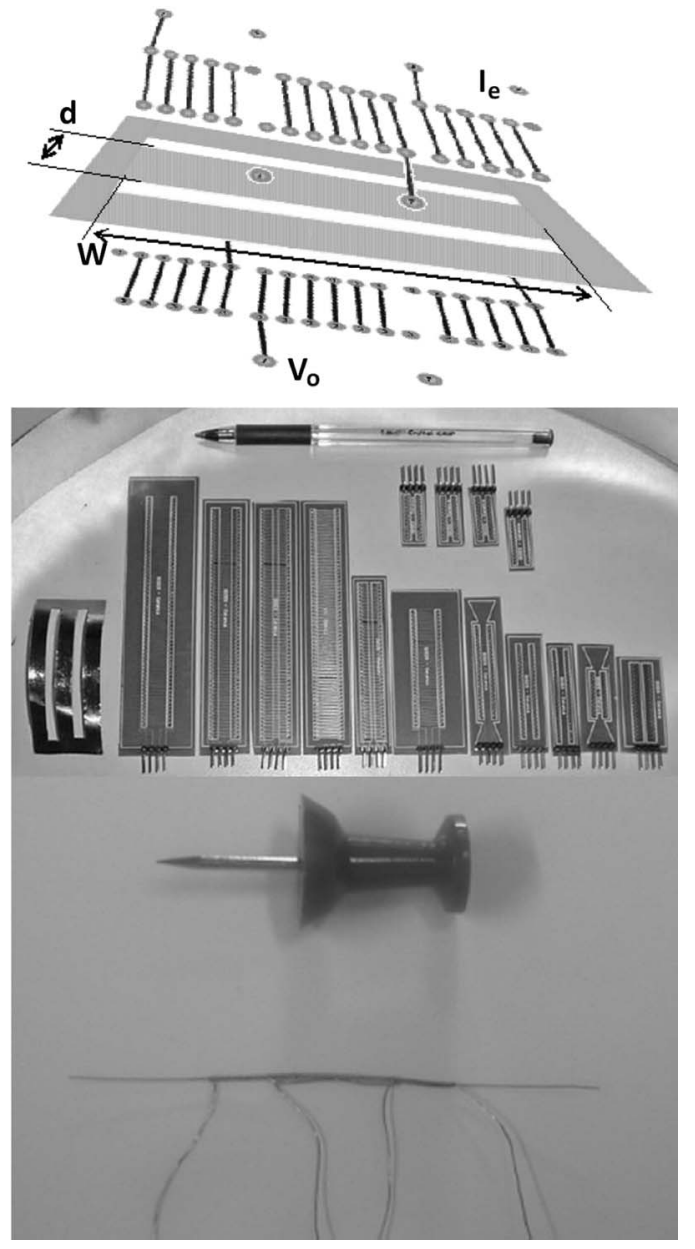

Fig. 7. (Top) Drawing of the fluxgate structure realized via a modified PCB technology. (Middle) Photograph of the fluxgate fabricated by using the magnetic ribbon. (Bottom) Photograph of the fluxgate fabricated by using the 100- $\mu \mathrm{m}$ magnetic microwire.

sensors, the resolution is computed inside the shield. Hence, it is a function only of the sensor and its noise floor. It is independent of the operating environment of the sensor when used in an application, and the threshold. Hence, it is a number that can be used to characterize the sensor performance, much like the noise-floor characterization that is usually used for magnetometers that are read out via the response PSD. We will return to the resolution later in this paper; at the end of the next section, we describe how this quantity is obtained through experiments.

\section{E. RTD Fluxgate Fabrication: "Ribbon" and "Microwire"}

Several sensor prototypes have been fabricated to demonstrate the suitability of the RTD readout methodology, and different technology solutions have been explored for this task. In particular, the attention has been focused on the choice of the core material, which is required to have very high permeability and very low coercivity in order to satisfy the hypotheses made previously (e.g., instantaneous switching that can result in a nearly digital device). Two major solutions have been identified: one based on a "ribbon" magnetic core [68] and the other based on a magnetic microwire [69]. In Fig. 7, we 


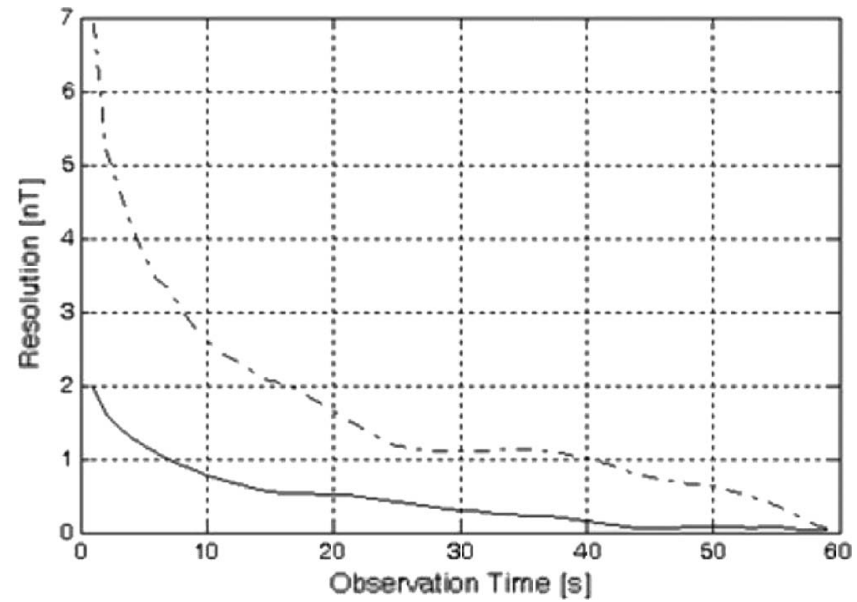

Fig. 8. Comparison of experimentally obtained resolutions for the (dashed curve) PCB and (solid curves) microwire fluxgate prototypes. The devices were driven by a triangular excitation signal of $5-\mathrm{mA}$ amplitude at $80 \mathrm{~Hz}$ (figure reproduced with permission from [43]).

show (top) the "skeleton" of the fluxgate, together with (middle) the ribbon material produced by Metglas, and (bottom) the microwire fluxgate prototype. These experimental sensor prototypes have been characterized in terms of performance [43], spatial resolution [70], demagnetization [71], and perming [72]. In Fig. 8, the results obtained during the experimental evaluation of the resolution of the two sensor releases as a function of the the time window length over which the output is averaged are shown. It is clear that the microwire fluxgate shows better resolution performance, and this is mainly due to the absence of damage to the core material magnetic domains; therefore, it is the best candidate when one wants to detect very weak magnetic signals. As shown in the Fig. 8, a resolution [66] of a few hundreds of picotesla has been obtained for these devices when averaging over a time window of $30 \mathrm{~s}$. The results shown here have been obtained by using an excitation current of $5 \mathrm{~mA}$ at $80 \mathrm{~Hz}$.

A careful choice of the system parameters leads to even better performances both in terms of resolution and power budget. More recent, carefully optimized experimental devices have yielded resolutions around $100 \mathrm{pT}$, at $1 \mathrm{~mA}$ and $320 \mathrm{~Hz}$ [64]. In Fig. 9(a), we show one of the most recent results obtained for the SCFG: the PSD, expressed in $\mathrm{pT} / \sqrt{\mathrm{Hz}}$, of the magneticfield response in the absence of the dc target signal. From the PSD, one can quantify the noise floor at $1 \mathrm{~Hz}$ of the device; it is around $100 \mathrm{pT} / \sqrt{\mathrm{Hz}}$. This value represents the amplitude of the "apparent" noisy external magnetic signal when the sensor is placed in a clean and quiet (magnetic) environment corresponding to a nominally "zero" target signal. It is prudent to reiterate that, in our mode of operation (via the RTD rather than the PSD) of the device, the bias signal is applied at relatively low frequency $[320 \mathrm{~Hz}$ for the device characterized in Fig. 9(a)], compared to what would be the case for a standard commercial device readout via the PSD (in this case, the bias frequency is typically around $10^{4} \mathrm{kHz}$ ). In the RTD approach, the optimal bias will always be a low-frequency and small-amplitude signal, thus significantly reducing the power consumption of our fluxgate. However, increasing the bias frequency to $320 \mathrm{~Hz}$ yields faster responses than those shown
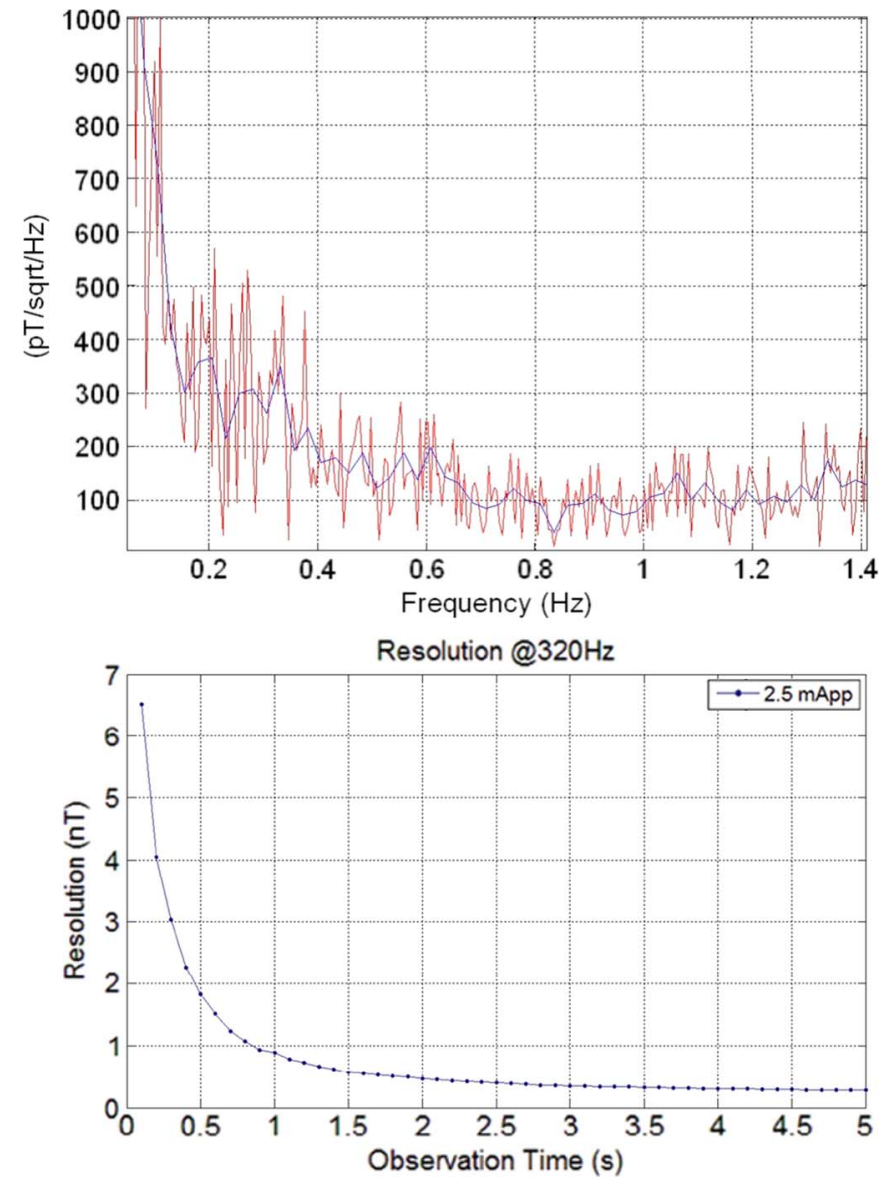

Fig. 9. (a) PSD (divided by the sensitivity, i.e., the ratio $\langle\Delta t\rangle / H_{x}$ ) of the response in an SCFG driven at $320 \mathrm{~Hz}$; the data are smoothed to better evaluate the noise floor at $1 \mathrm{~Hz}$. (b) Resolution experimentally obtained for the SCFG as a function of the time frame over which the output data are averaged (observation time); resolution values well below $1 \mathrm{nT}$ are obtained with increasing observation times (Fig. 9(b) reproduced with permission from [64]).

in Fig. 8; in fact, in Fig. 9(b), the resolution (37) is plotted against the observation time. The figure shows that resolution values (below $1 \mathrm{nT}$ ) are obtained with a 1-s observation time. In particular, the PSD shown in Fig. 9(a) has been obtained by using an observation time of $300 \mathrm{~ms}$, where the resolution is around $2.5 \mathrm{nT}$; better resolution values will require longer observation times, of course.

The aforementioned performance, taken together with the very low cost of the device and, also, the inherent simplicity of the device and of its conditioning circuit, puts RTD fluxgates at, or near, the forefront of the current state of the art [32], [35], [38]. Our results make clear that the tradeoff between resolution and observation time is the ultimate decider of the efficiency and viability of these sensors in practical operating scenarios.

\section{CCFG MAGnEtometeR}

While unforced dynamical systems of the general form (2) do not oscillate, by coupling $N>2$ elements of the general form (1) in a specific configuration and by ensuring that the initial state of at least one of them is different from the others, the system dynamics transit to oscillatory behavior [25], [26] when 
a suitably chosen control parameter exceeds a critical value; this control parameter can be either the coupling coefficient or a dc applied signal. We now focus our attention on a real device that exploits these properties (with a specific topology that is believed to occur in some biological systems).

As discussed in the introduction, the idea is to couple an odd number $N$ of wound ferromagnetic cores in a ring configuration, i.e., the coupling is unidirectional and the boundary conditions are cyclic; this means that the bias signal needed to switch the $i$ th element (i.e., to have its state point transition its energy barrier) will be provided by the output of the one that it is coupled to. This affords the possibility of being able to generate the reference oscillations, needed in the case of the SCFG, in the circuit itself, without the necessity of the externally applied bias signal. In turn, this could result in a reduced noise floor since, in the single-core magnetometer, the bias signal generator accounts for a significant percentage of the noise floor.

This model realization of a "CCFG magnetometer" can then be described via the following equations [25], [26] for $N=3$ coupled cores:

$$
\begin{aligned}
& \dot{x}_{1}=-x_{1}+\tanh \left(c\left(x_{1}+\lambda x_{2}+H_{x}\right)\right) \\
& \dot{x}_{2}=-x_{2}+\tanh \left(c\left(x_{2}+\lambda x_{3}+H_{x}\right)\right) \\
& \dot{x}_{3}=-x_{3}+\tanh \left(c\left(x_{3}+\lambda x_{1}+H_{x}\right)\right)
\end{aligned}
$$

where $x_{i}(t)$ represents the (suitably normalized) core magnetization of each unit and $H_{x}$ is the external dc magnetic field to be sensed. The parameter $c$ has already been defined in the context of (2), while the time constant $\tau$ has been set equal to unity for convenience. It is important to note that the oscillatory behavior occurs even for $H_{x}=0$; however, when $H_{x} \neq 0$, the oscillation characteristics change, and these changes can be exploited for signal quantification purposes. The elements in (38) are assumed to be identical for theoretical simplicity; however, in practice, the cores and circuit elements are not the same, and this situation has been addressed in [73]. Notice that the unidirectional coupling term, having strength $\lambda$, which is assumed to be equal for all three elements, is inside the nonlinearity, which is a direct result of the mean-field nature of the description.

Under the aforementioned conditions, the system (38) displays an oscillatory behavior [25], [26] which commences when the coupling coefficient exceeds a threshold value

$$
\lambda_{c}=-H_{x}-x_{\mathrm{inf}}+\frac{1}{c} \tanh ^{-1} x_{\mathrm{inf}}
$$

with $x_{\text {inf }}=\sqrt{(c-1) / c}$; note that, in our convention, $\lambda<0$, so that oscillations occur for $|\lambda|>\left|\lambda_{c}\right|$. The individual oscillations (in each elemental response) are separated in phase by $2 \pi / N$ and have period [25], [26]

$$
T_{i}=\frac{N \pi}{\sqrt{c x_{\mathrm{inf}}}}\left[\frac{1}{\sqrt{\lambda_{c}-\lambda}}+\frac{1}{\sqrt{\lambda_{c}-\lambda+2\left|H_{x}\right|}}\right]
$$

which shows a characteristic dependence on the inverse square root of the bifurcation "distance" $\lambda_{c}-\lambda$, as well as the target
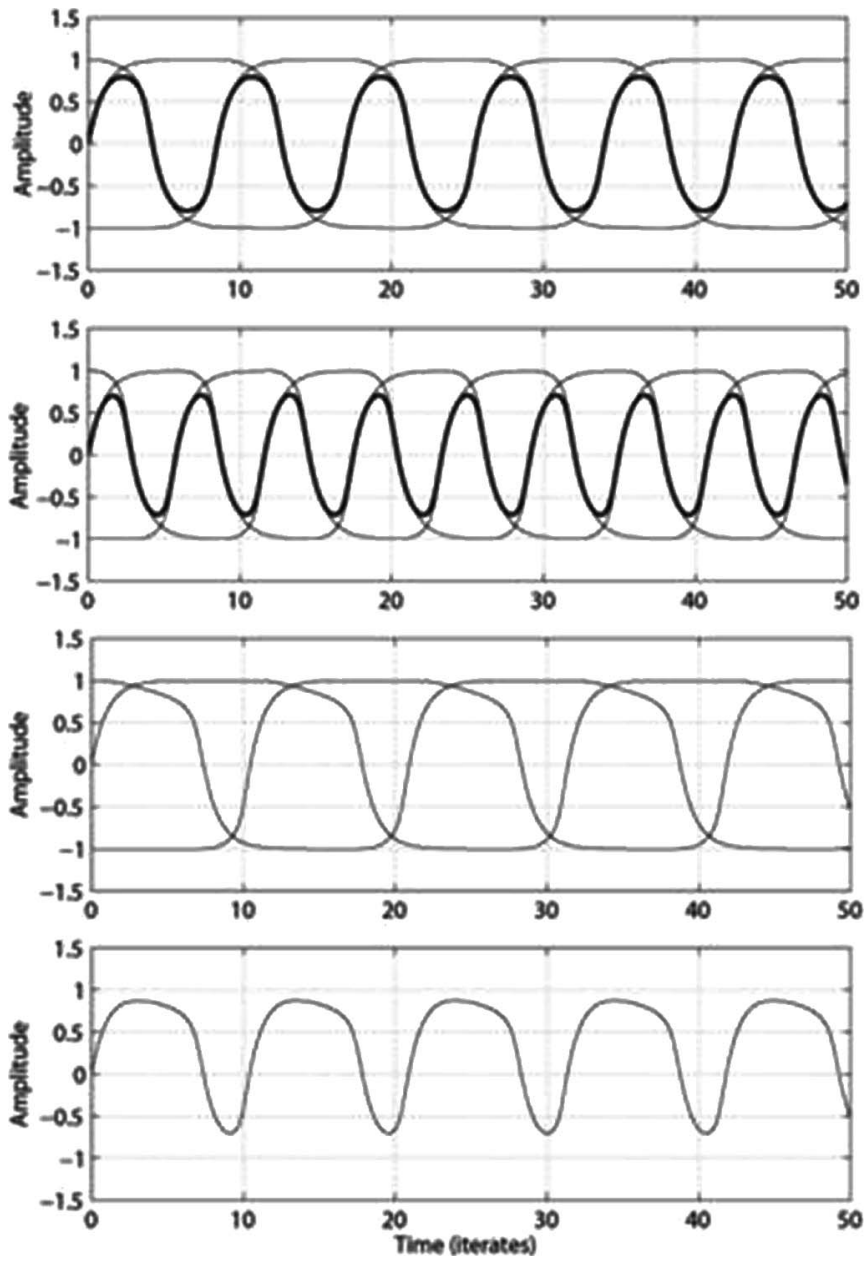

Fig. 10. Emergent oscillatory behavior in the coupled system (38) for $N=3$. The top panel shows the oscillations near the critical point. The summed response is indicated by the thick black lines, and the individual element responses follow the gray lines in all panels. The amplitudes are fully grown at the start of the bifurcation, and the frequency is low. At the birth of the oscillations, the frequency is zero. The parameters are set at $\lambda=-0.60$ and $H_{x}=0$. The second panel shows the oscillations for a higher coupling strength $\lambda=-0.75$, and $H_{x}=0$. Contrasted with the top panel, the frequency increases significantly. The frequency scales as a square root of $|\lambda|$ and $H_{x}$. The third panel shows the individual element oscillations for $\lambda=-0.60$ and $H_{x}=0.05$. Notice that the sum signal (last panel), obtained from the individual oscillations in the third panel, is greatly offset between the upper state (above zero) and the lower state (below zero). Also notice the decrease in frequency when the target signal $H_{x}$ is nonzero compared to the top panel. The initial conditions for all simulation runs are $\left(x_{1}, x_{2}, x_{3}\right)=(1.0,0.0,-1.0)$ and $c=3$, and the time step size is 0.00268 . For each panel, the critical coupling $\lambda_{c}$, at the onset of the oscillations, may be determined from (39) (figure reproduced with permission from [25]).

signal $H_{x}$; these oscillations can be experimentally produced at frequencies ranging from a few hertz to high kilohertz. The summed output $\sum_{i} x_{i}(t)$ oscillates at period $T_{+}=T_{i} / N$. Note that the "oscillations" are actually switching events between the stable states of each core; they occur as long as at least one element has an initial condition that is different from the remaining elements (clearly, in any practical system, this condition is almost always satisfied). The setup clearly eliminates the need to apply the reference bias signal, as required for the single fluxgate. Increasing $N$ changes the frequency of the individual elemental oscillations, but the frequency of the summed response is seen to be independent of $N$. Fig. 10 shows 


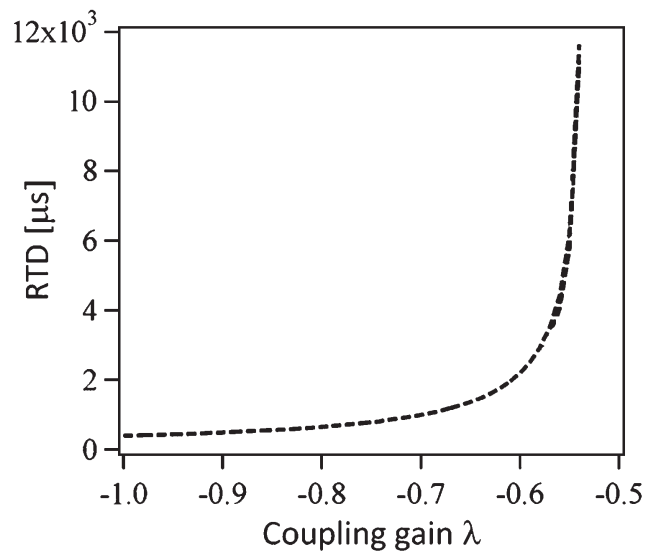

Fig. 11. Response curve of the CCFG to an applied target dc magnetic field $H_{x}$ versus coupling strength. As $\lambda$ approaches the critical value $\lambda_{c}=$ -0.5345 , the response curve rises almost vertically, which suggests that the sensitivity of the device increases dramatically in this regime. $c=3, H_{x}=$ 0.1 , and $-1.0 \leq \lambda \leq-0.54$. The RTD $(\Delta T)$ response is plotted (figure reproduced with permission from [25]).

the oscillatory behavior, obtained via numerical simulations of the coupled system (38). The RTD for this system can be computed, in the absence of a noise floor, as [25], [26]

$$
\Delta T \approx \frac{\pi}{\sqrt{c x_{\text {inf }}}}\left[\frac{1}{\sqrt{\lambda_{c}-\lambda}}-\frac{1}{\sqrt{\lambda_{c}-\lambda+2\left|H_{x}\right|}}\right]
$$

which vanishes (as expected) for $H_{x}=0$, and can be used as a quantifier of the target signal, which is analogous to the time-domain operation of the SCFG. Using the last expression, we can obtain an approximation to the RTD in the small $H_{x}$ limit

$$
\Delta T \approx \frac{\pi H_{x}}{\sqrt{c x_{\mathrm{inf}}}}\left(\lambda_{c 0}-\lambda\right)^{-3 / 2}
$$

where we set $\lambda_{c 0}=\lambda_{c}\left(H_{x}=0\right)$. We observe that the sensitivity $\partial(\Delta T) / \partial H_{x}$ is significantly enhanced as we get closer to the critical point; this is shown in Figs. 11 and 12. It is worth reiterating that a sensitivity $\partial T_{+} / \partial H_{x}$, defined via the (summed) oscillation period $T_{+}$, is actually proportional to $H_{x}^{2}$ (for small $H_{x}$ ), as can readily be calculated from (40). This may not be desirable in practical sensors where one would like to develop the optimal sensor configuration independently of the target signal. Hence, from this standpoint, the RTD constitutes the more desirable measure.

The system sensitivity, defined previously, is seen to significantly increase when the critical point in the oscillatory regime is approached; this suggests that careful tuning of the coupling parameter so that the oscillations have very low frequency could offer significant benefits for the detection of very small target signals. The preceding statement must, however, be qualified by an important caveat: In practical setups, the oscillation frequency cannot be set too low in order to ensure good coupling between the cores and the circuit components; in turn, this places an upper bound on the sensitivity. In addition, sensor noise (both from the electronics and the core material) is likely to be somewhat larger in amplitude at low frequencies. Despite these caveats, however, it is clear that our coupling scheme affords the exploitation the target-signal dependence

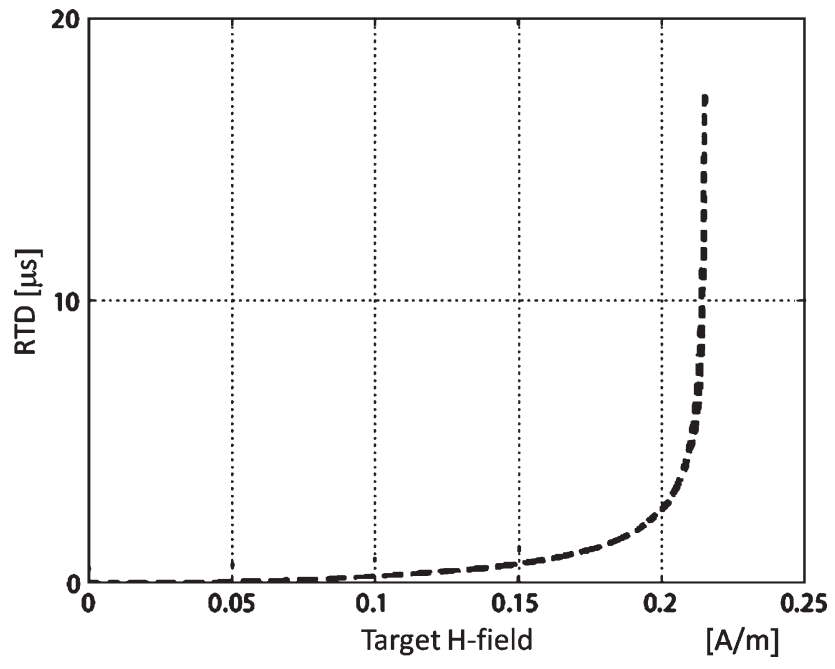

Fig. 12. RTD plotted as a function of the (weak) de target signal $H_{x}$ with a fixed coupling parameter $\lambda=-0.8$. The sensitivity is now given by the slope of the response curve and is seen to increase as $H_{x}$ approaches the potential energy barrier height, as expected (figure reproduced with permission from [25]).

of the emergent oscillations for detection and quantification purposes.

A recent twist [74] to the aforementioned coupling scheme has led to a substantial improvement in the performance of the CCFG. The idea is to reverse the orientation of successive cores so that the sign of the $H_{x}$ term in (38) alternates; for an odd $N$, this guarantees that there will be two adjacent elements with the same sign of $H_{x}$. This alternating configuration [alternate output (AO)] arrangement is described (with $i=1, \ldots, N$, where $N$ is odd) by

$$
\dot{x}_{i}=-x_{i}+\tanh \left(c\left(x_{i}+\lambda x_{i+1 \bmod N}+(-1)^{i+1} H_{x}\right)\right) .
$$

One can readily calculate [74] the oscillation period $T_{i}{ }^{A O}$ of an individual element, as well as the threshold value $\lambda_{c}^{A O}$ for the onset of oscillations; these quantities are identical to those given previously for the conventional arrangement. However, this does not apply to the RTD. We find that this quantity changes, depending on the particular element under consideration. Focusing on the element $i=1$, which has the same sign of $H_{x}$ as the element that it is back coupled to (namely, the $i=3$ element), we find [74]

$$
\Delta T_{1}=N \Delta T
$$

with the result generalized to the arbitrary $N$ (odd) case. In (44), the quantity $\Delta T$ represents the RTD obtained via the elements $N=2$ or 3 ; in turn, we can show that $\Delta T$ is the same result that would accrue in the standard configuration [standard output (SO)], i.e., using the $\mathrm{AO}$ and carefully selecting the appropriate element whose RTD response is computed provide enhanced sensitivity to a given target dc signal. This departure from the standard behavior in the arrangement (38) is evident in Fig. 13. In fact, recalling that $\partial \Delta T / \partial H_{x}$ measures the sensitivity of a CCFG, it follows that the sensitivity of the AO configuration improves linearly by a factor of $N$ when compared to the best sensitivity that can be achieved by the 

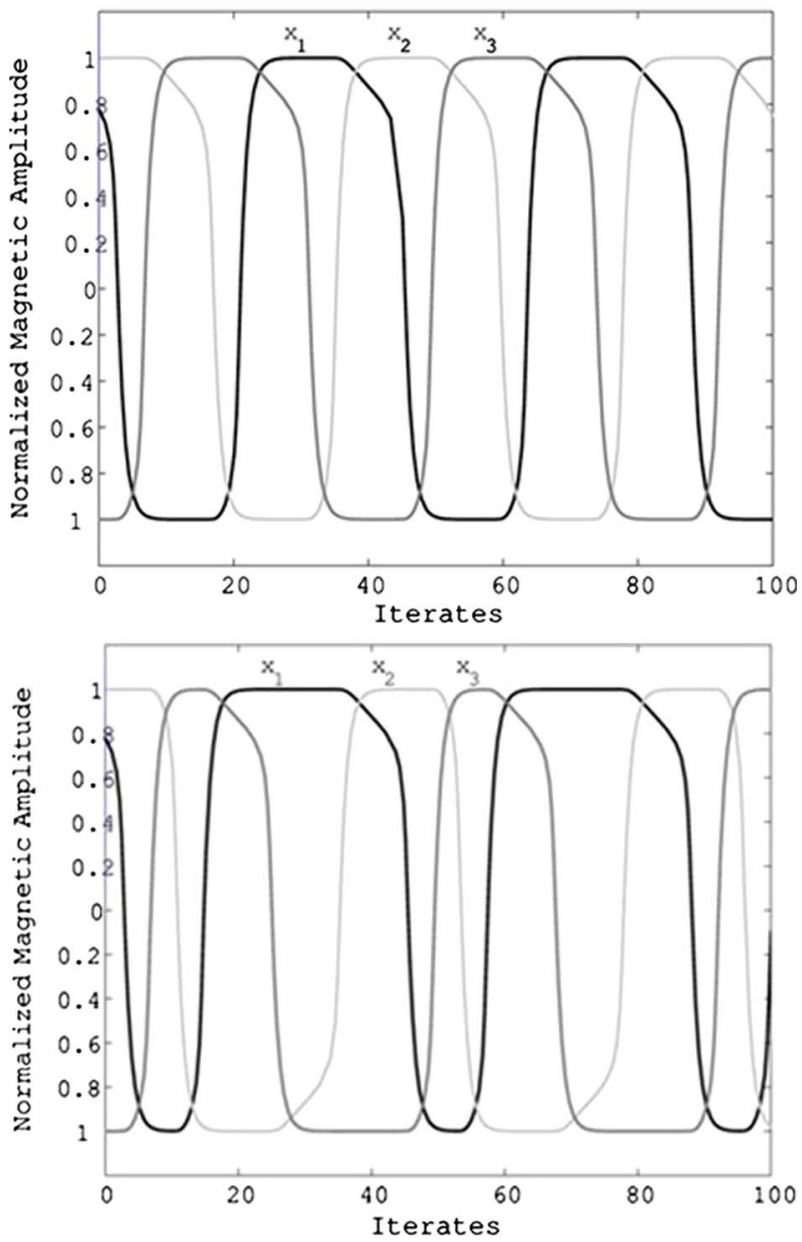

Fig. 13. Time series from simulations on (above) a standard CCFG arrangement and (below) an AO arrangement. The RTD $\Delta_{1}(t)$ (see text) of the element $x_{1}$ in the $\mathrm{AO}$ arrangement is seen to be $N$ times the corresponding quantity in the standard arrangement. $N=3, c=3, \lambda=-0.54$, and $H_{x}=0.07[\mathrm{~A} / \mathrm{m}]$ (figure reproduced with permission from [74]).

standard CCFG configuration, given the same external signal and core parameters. For best performances, the output signal considered is the one gathered at the "inverted" core.

The dependence of the RTD and, consequently, of the sensitivity on the size (i.e., number of coupled dynamic elements) of the ring in the $\mathrm{AO}$ configuration is in direct contrast to the sensitivity response of the standard configuration, in which increasing $N$ beyond $N=3$ does not lead to additional benefits. The aforementioned observations are confirmed in Fig. 14, in which we calculate numerically the RTD for a CCFG system with standard (SO), as well as alternating (AO), configuration. As expected, near the onset of coupling-induced oscillations, the RTD response of the $i=1$ element in a standard CCFG configuration remains constant, while that of the $\mathrm{AO}$ configuration increases linearly with $N$.

\section{A. CCFG Magnetometer Implementation}

The experimental coupled-core device involves three wound coupled cores (similar to those used in the SCFG) and the coupling circuit that connects them together to conform to the model equation (43). Fig. 15 shows the block diagram, as an
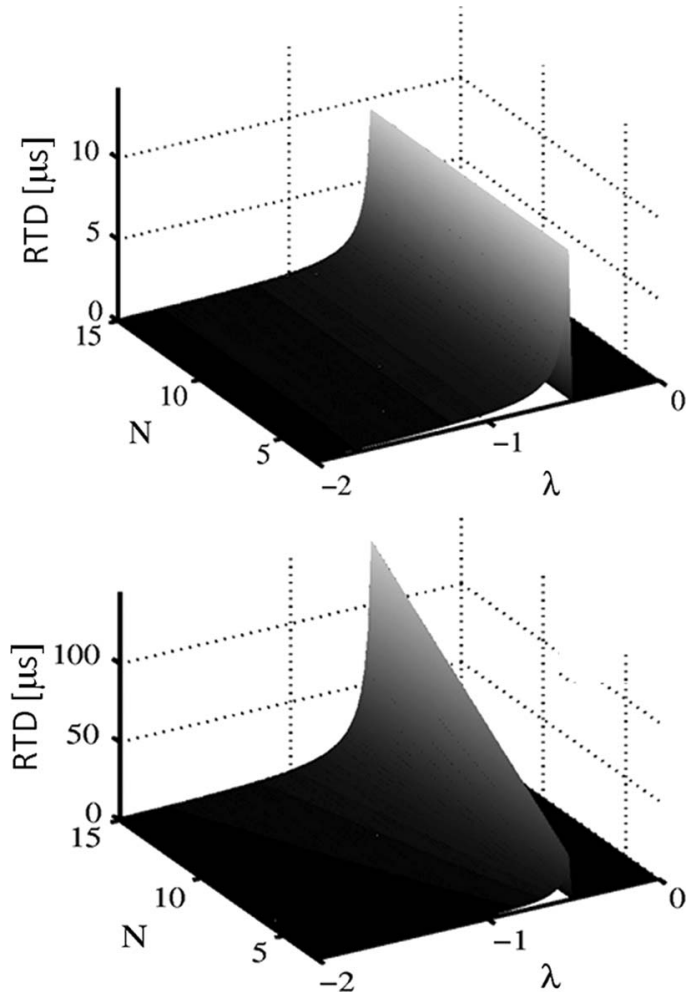

Fig. 14. RTD response $\left(\Delta T_{1}\right)$ of the $x_{1}$ element in a CCFG as a function of the number of elements $N$ in the ring and coupling strength $\lambda$. (Above) Standard configuration and (below) AO configuration. Near the onset of coupling-induced oscillations, in particular, the RTD response of the standard configuration remains constant (as expected), while that of the AO configuration increases linearly as a function of $N . c=3, H_{x}=0.07$ a.u. In each case, the maximum RTD is realized infinitesimally past the critical coupling $\lambda_{c}$. The response has been computed, in both figures, via a numerical integration of the full system dynamics (38) and (43). Note the (substantial) difference in the vertical scales (figure reproduced with permission from [74]).

overview, of the setup. The cores are mounted on the faces of a structure with a triangular section for orienting all of them in the same direction (the device is effectively a single-axis sensor with the target field measured along each core axis) and then coupled through electronic circuits wherein the voltage readout (i.e., the time derivative signal of the flux detected by one of the sensing coils) is amplified by a voltage amplifier. Next, the signal is passed through an integrator to convert the derivative signal seen by the sensing coil back to the "flux" form so that the experimental system closely conforms to the model. The signal then passes through an amplifier to achieve adequate gain to drive the adjacent fluxgate. Following this, the signal passes through a voltage-to-current converter $(V-I$ converter) in its final step to drive the primary coil of the adjacent fluxgate. The setup is repeated for the other two coupling connections for the remaining cores, and all values of the coupling circuit parameters are closely matched from one set to the other. Each stage of the coupling circuit also employs high-speed and high-precision operational amplifiers (op-amps) to minimize the time delay in order to conform closely to the model, since the knowledge of the state variable $x_{i}$ must be available instantly in any application. Once the coupled-core device is configured, as described previously, the outputs of the three stages are sent for processing in order to extract the information about the target field being detected. 


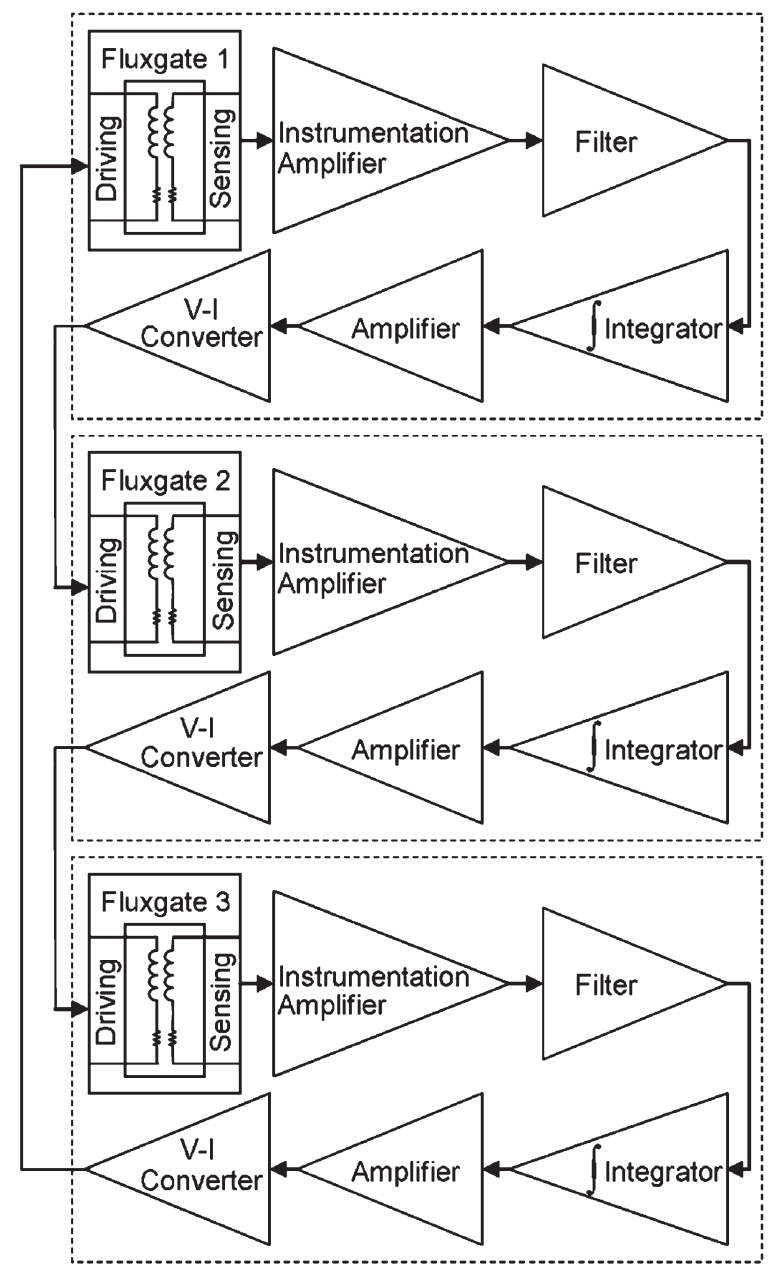

Fig. 15. Flow diagram of the coupled system as an overview for the device realization (figure reproduced with permission from [25]).

The signals are taken from each of the integrator stages and passed through a summing circuit; the output of the summing circuit is then passed through an ST. If the signal is greater than the upper threshold value of the ST, then the output is a HIGH, corresponding to $+10 \mathrm{~V}$. The output remains HIGH until the input signal falls below the lower threshold value of the ST, which then outputs a LOW, corresponding to $-10 \mathrm{~V}$. In essence, the ST converts the output into a clean dichotomous signal, retaining only the all-important information about the locations (along the time axis) of the switching events. The output from the ST is passed through a voltage divider and a diode to convert the signal to transistor-transistor logic in which $+5 \mathrm{~V}$ corresponds to a HIGH and $0 \mathrm{~V}$ corresponds to a LOW. These HIGH and LOW states are then passed to a programmable integrated circuit microcontroller for further processing. Then, the residence time is determined as follows: The lower residence time $\left(T_{-}\right)$is the time difference between the crossing time of the upper threshold and the crossing time of the previous lower threshold; the upper residence time $\left(T_{+}\right)$ is the time difference between the crossing (in the upward direction) time of the lower threshold and the next crossing (in the negative direction) of the upper threshold. Clearly, the rapid time constant $\tau_{F}$ ensures that the crossing events are near instantaneous.
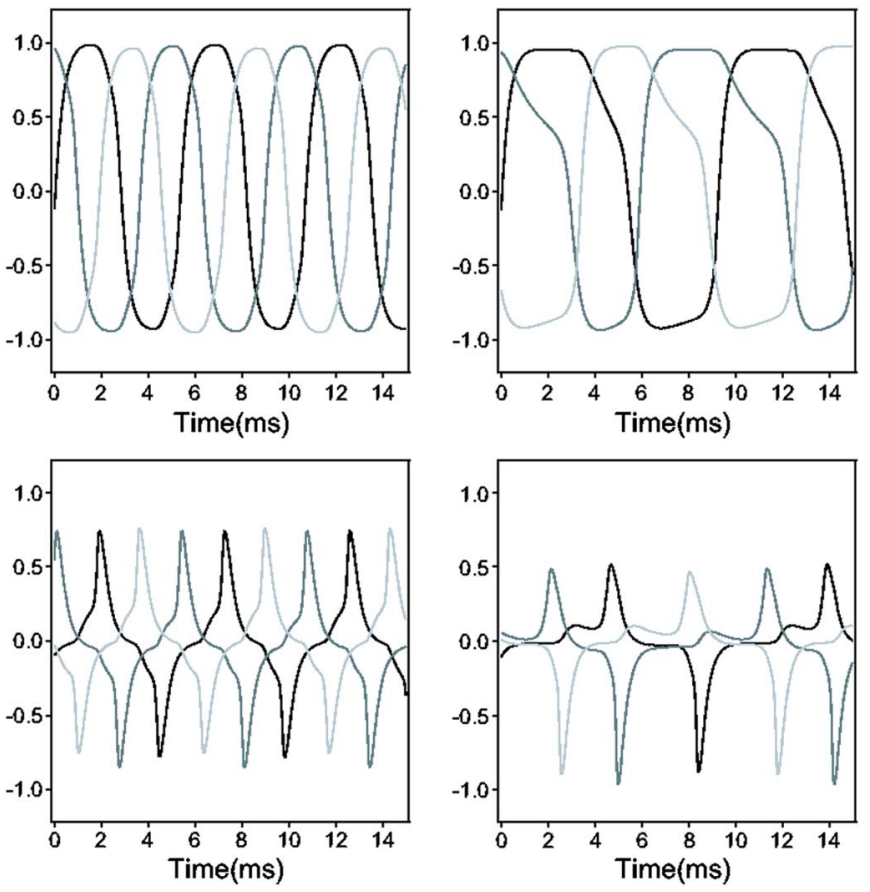

Fig. 16. Time series from (left column) the experimental CCFG system for $H_{x}=0$ and (right column) a small dc target signal $H_{x}=6.0 \mathrm{~A} / \mathrm{m}$. The top panel shows the traveling wave pattern, illustrating the oscillation of the three coupled fluxgate cores. Each one has the same amplitude and frequency, but each is phase shifted by $120^{\circ}$, as observed in the numerical model. The bottom panel shows the outputs as seen by the sensing coils of the coupled device. The response matches the numerical observations (Fig. 10) (figure reproduced with permission from [26]).

The oscillations observed from the experimental prototype are in very good qualitative agreement with the theoretical expectations (Fig. 16). The system readily oscillates in a traveling wave pattern, and each wave is phase shifted by exactly $2 \pi / 3$, as predicted by the model. The comparison of the oscillations from the experiment to the numerical results shows good agreement with the caveat that, since the values of $c$ and the time constant $\tau$ in the actual device (we set $\tau=1$ in the model for convenience) are not known, we cannot correctly compare the timescales in the model and the experimental observations. The amplitudes of the oscillations in the experiment are also arbitrary in comparison to the model because the recorded voltages depend on the gains set in the coupling circuit. On the other hand, the magnetic flux in the model saturates between \pm 1 , but in the devices, this quantity cannot be measured directly.

Further illustration of good agreement between the numerical and prototype systems is the frequency scaling (Fig. 17), as a function of the coupling strength and also as a function of the applied (dc) field. As expected from (40), the frequency of the coupled sensor system should rise as the square root of the coupling strength or the applied field magnitude. As the coupling strength increases, the frequency decreases until the coupling strength is at the critical value, where the oscillations cease to exist. Increasing the coupling values beyond this point will not produce any oscillation. We recall that, in the convention adopted in the theoretical description of this system, the coupling is negative, so that an "increase" of coupling implies that the coupling coefficient $\lambda$ approaches zero. Similarly, increasing the applied field in either direction away from zero 

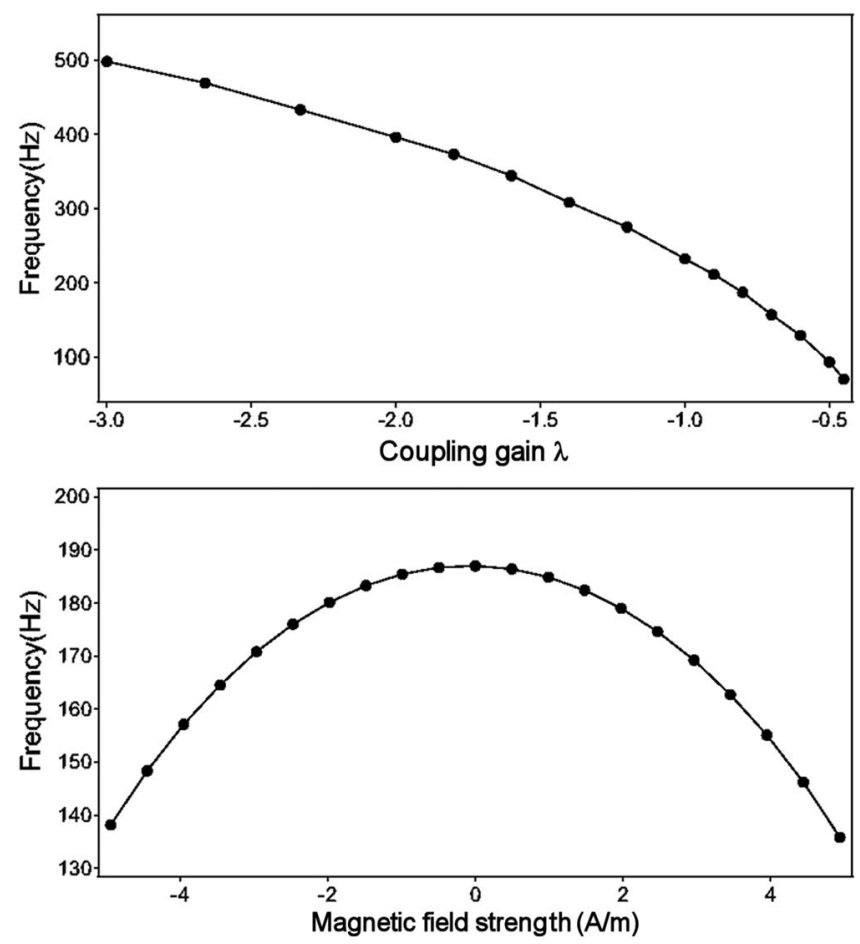

Fig. 17. Experimentally obtained frequency scaling with respect to the coupling strength, expressed as a gain, and the applied dc target magnetic field. The curves scale as $\sqrt{\lambda_{c}-\lambda}$ in accordance with the theory.

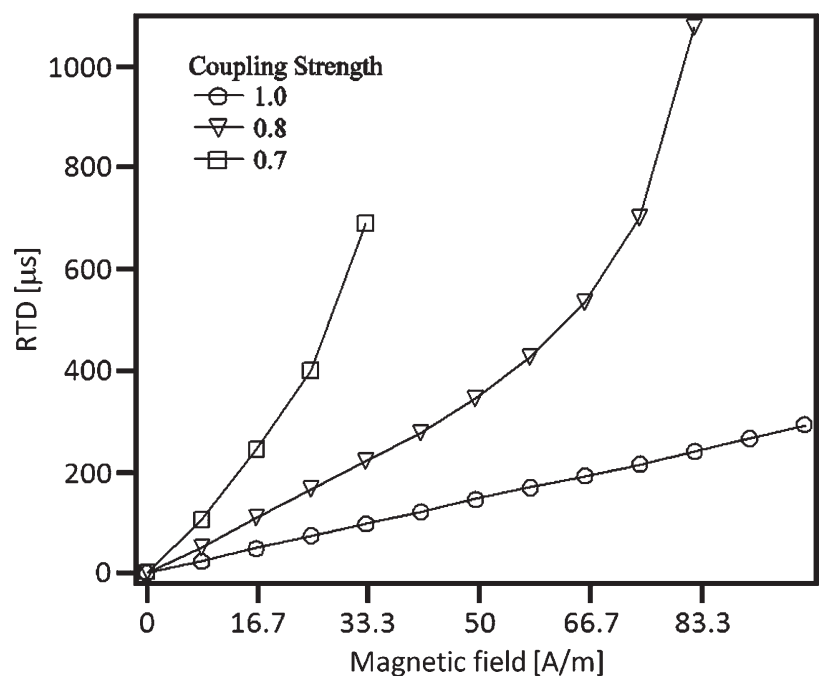

Fig. 18. Experimentally obtained family of responsivity curves, in terms of RTD as a function of the applied field for different coupling strengths (figure reproduced with permission from [26]).

will reduce the frequency of the oscillations, as predicted by the model. The oscillations cease to exist when the applied field is too large, because the field moves the system past the critical point of the onset of the oscillation. In fact, for a given coupling strength, the maximum detectable field magnitude is equal to the separation between the coupling strength and the critical value.

Fig. 18 shows a family of responsivity curves as a function of the applied field for different coupling strengths; the figure should be compared to the theoretically generated Fig. 12. The slopes of the curves indicate how responsive the coupled system

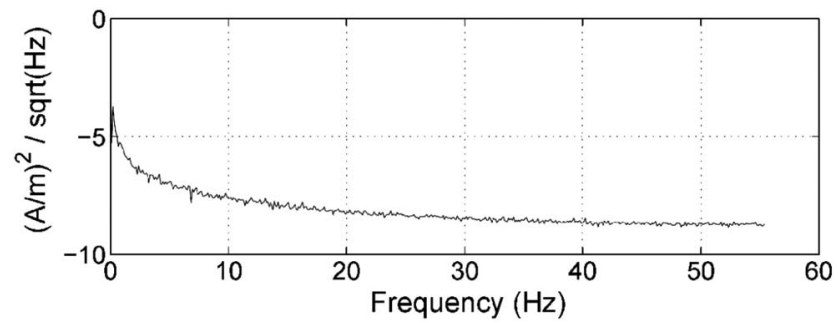

Fig. 19. Power spectrum of the moving-window averaged coupled-core magnetometer output in the $0.003-55.395-\mathrm{Hz}$ frequency range.

is to the applied field. As the coupling strength is increased toward the critical value, the responsivity curve becomes steeper. The greatest sensitivity is realized when the coupling strength is set closest to the critical value, but in this regime, it can only detect a very small target field amplitude. Hence, the ability to tune the coupling to detect a range of target field strengths must be a central feature of this mode of operation; when implemented, it presents a capability where one may tune the excitation amplitude to reduce the sensitivity and increase the operating range. The experimental sensitivity obtained for the laboratory prototype is $S=0.000531 \mathrm{~s} /(\mathrm{A} / \mathrm{m})$. We remark that $0.000531 \mathrm{~s}$ is the variation of the RTDs when a field of $1 \mathrm{~A} / \mathrm{m}$ is applied; this implies that, by using a $200-\mathrm{MHz}$ counter to estimate the $R T D$, the uncertainty from the counter alone would correspond to $10^{-5} \mathrm{~A} / \mathrm{m}$ or, referring to the magnetic flux in vacuum, to about $12 \mathrm{pT}$. Of course, a faster clock can alleviate this resolution limitation of the device, but that is not the point. The result must be viewed in context with the fluctuations in the RTD produced by magnetic and $\mathrm{E}$ noises; the actual resolution of the device is indeed limited by the total system noise. In the experiments, the noise is estimated from a time series $(270 \mathrm{~s})$ of the magnetometer output with the sensor placed in a three-layer Metglas [68] magnetic shield, with no target field applied (Fig. 19).

\section{B. Cooperative Behavior for Increasing N: Mitigating the Effects of Background Noise}

The noise floor in the CCFG (and the SCFG) can arise from a contamination of the external target signal, as well as from the readout electronics, magnetic core, etc. Regardless of the source of the noise, we have treated it as being Gaussian band-limited noise, as already formally defined in (15).

A small additive noise floor (arising from internal sources) manifests itself in fluctuations of the "rest" states of each core, about the deterministic mean values $\pm b$ (the minima of each core potential energy function in the absence of coupling). The numerical simulations [75] of the coupled system, for this case, show that the threshold crossings are quite sharp and unambiguous in the presence of this noise term, as long as the noise is not too strong; this is a direct consequence of the very low time constant $\tau$ (usually, $\ll \tau_{c}$ ) of each core; to all intents and purposes, each element behaves like a static nonlinearity, with near-instantaneous switching events.

We now consider the situation wherein the target dc signal is noise contaminated. In this case, one augments additively the dc target signal with a correlated noise term $\zeta(t)$ [of the form 

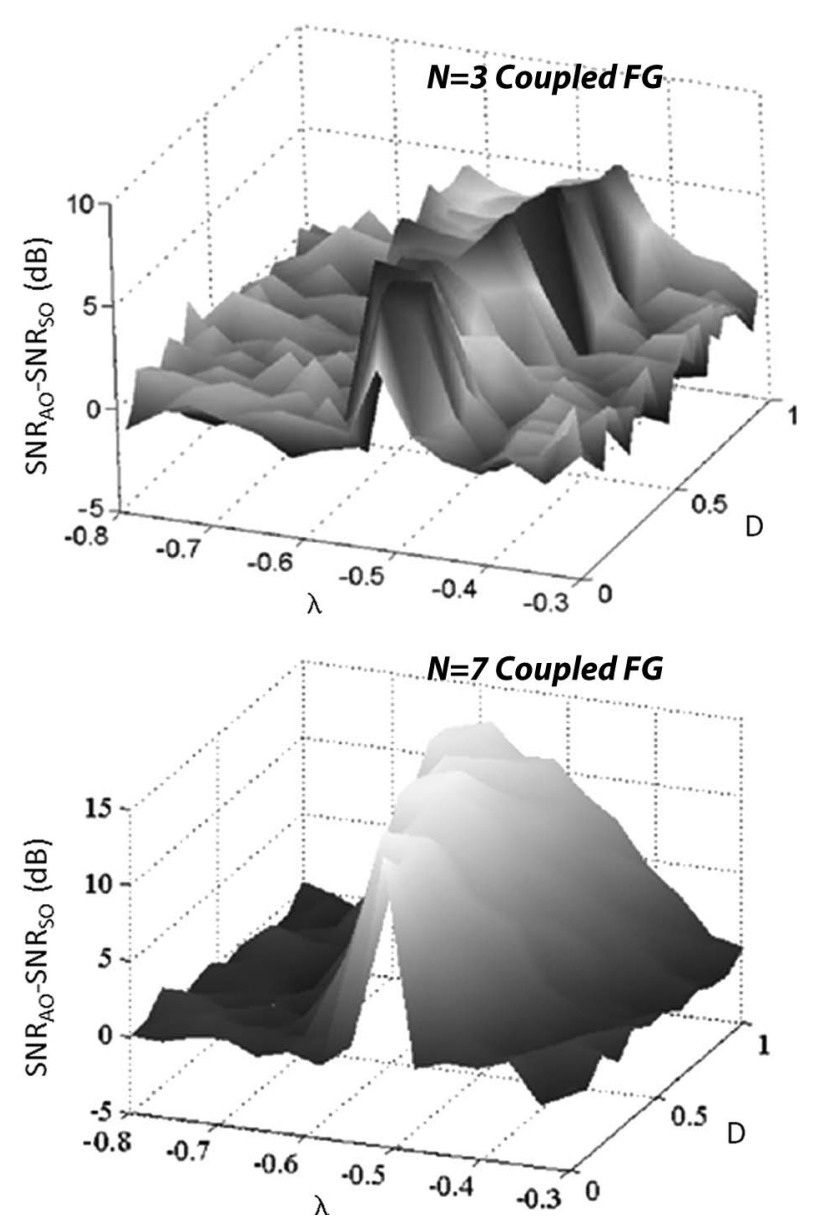

Fig. 20. Simulation results showing the difference in SNR responses between $\mathrm{AO}$ and $\mathrm{SO}$ configurations. The graphs are plotted against the coupling strength $\lambda$ and the noise parameter $D$ [see discussion after (15)]. Observe that, near the onset of coupling-induced oscillations, the SNR response of the AO configuration is significantly better. The maximum SNR in each case is obtained just barely past the critical point (defined by $\lambda_{c}$ ). The improvement in the SNR as a consequence of the increase in the number $N$ of coupled elements should be observed: Top figure refers to $N=3$, while bottom figure refers to $N=7$. Other parameters are $c=3, \tau_{c}=150.0$, and $\epsilon=0.07$. In each case, the SNR has been evaluated for the element with the maximal response (see text) (figure reproduced with permission from [26]).

specified in (15)] in (38) and (43). Our simulations in this case [74] have focused on a comparison of the spectral response (quantified by an SNR obtained at the spectral feature at the oscillation frequency in the oscillatory regime), the intention being to compare the response of the coupled system (38) with the one $(A O)$ where the sign inversion has been introduced in the coupling factor. These comparisons are well summarized in Fig. 20, wherein we plot the SNR difference (at the oscillation frequency) between the standard ( $\mathrm{SO}$ ) and alternating (AO) configurations (given by (38) and (43), respectively). One readily finds that the AO configuration provides far better SNR performance when operated close to the onset of switching, i.e., in the low-frequency oscillation regime just past the critical point. The improvement is enhanced for increasing $N$. The AO configuration therefore offers a tangible way of improving the sensor responsivity (or resolution) by exploiting large- $N$ effects.

The resolution $R$ for one element of the coupled-core arrangement can be computed from (37) [76]. This expression has been theoretically derived under the small noise approxima- tion. Using the AO arrangement and considering the "favored" element (which yields the best response) $x_{1}$, we arrive at the following:

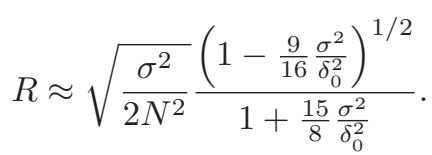

In deriving (45) [76], we have represented the noise as a contamination of the threshold or, equivalently, the dc target signal, with $\delta_{0}$ being the bifurcation distance $\left|\lambda_{c}-\lambda\right|$. The sensor noise floor is seen (in experiments) to manifest itself as fluctuations superimposed on the upper and lower thresholds given roughly by the inflection points of the underlying potential of the active element that is assumed to be isolated during the switch. Here, we insert an important conceptual point: The coupled system (38) is not derivable from the gradient of a potential energy function because of the unidirectional coupling. However, during a switching event, the switching element may be regarded as stemming from a single-body potential energy function since the remaining elements are in their steady states. This visualization of the sequential switching dynamics allows us to effectively decouple the dynamics during a switching event; in turn, we can derive [25], [26] the closedform expressions (39)-(41) for the deterministic system. The aforementioned approximation breaks down in the presence of large amounts of noise; in this case, one can no longer assume that two of the elements will remain confined to their steady states while the third element is switching.

The very small (compared to the other timescales in the system) sensor time constant guarantees that, once the switching event is underway (i.e., the state point has reached a switching threshold), the duration of the switch is near instantaneous and quite "clean," i.e., there are no fluctuations about the unstable saddle point. For very large noise, this description breaks down, of course; however, practical sensors are specifically engineered so that the sensor noise floor is extremely low. Under the same assumptions, the RTD density can also be derived [76]; as expected, this density has a noise-dependent tail which gets longer (i.e., the mode of the density function shifts to smaller values) with increasing noise intensity. For very weak noise intensity, the tail shrinks with the density function approaching a Gaussian; this has also been corroborated in our laboratory experiments. We note that the resolution is independent of the target signal $H_{x}$ and improves with increasing $N$. In practice, however, increasing the number of elements adds to the engineering complexity and also results in a higher noise floor due to the additional cores and circuit elements, together with an increased power budget; hence, one always must strike a fine balance between these issues and the resolution that might be appropriate for a particular application.

\section{Experimental Measurement of the CCFG Resolution}

We conclude this section with a brief description of how the resolution (37) is measured experimentally in a CCFG consisting of $N=3$ cores, following the AO dynamics (43), i.e., the "favored" element for measuring the RTD is $x_{1}(t)$. The 
procedure parallels that used in the SCFG to obtain Figs. 8 and 9 . We use an observation time (recall that this is the interval at which our measurement window is updated) and an oscillation frequency that is adjusted (via the coupling $\lambda$ ) to yield $15-20$ cycles of the response during this observation window. Of course, the observation time can be increased, but this would depend on the circumstances of the particular application and, more importantly, on the statistics and stationarity (or lack thereof) of the ambient noise. Keeping $H_{x}$ fixed, we compute the time-averaged RTD $\Delta_{1}$ by averaging the RTDs obtained in the observation window. The experiment is then repeated several times for the same $H_{x}$; each repetition yields a time-averaged (over the observation window) RTD which is not necessarily the same as the others due to fluctuations. In this way, one obtains a large number of time-averaged RTDs corresponding to the fixed value of $H_{x}$. The quantity $\left\langle\Delta_{1}\right\rangle$ is then the statistical average of these points (for the same value of $H_{x}$ ). The process is repeated for different values of $H_{x}$. A plot of $\left\langle\Delta_{1}\right\rangle$ versus $H_{x}$ shows clusters of discrete points (each point corresponding to an average over the observation window) for each value of $H_{x}$. The locus of the statistical means of each cluster of points then yields a straight line for small $H_{x}$. In Fig. 21, we have shown the "return map" of the (experimentally obtained) RTDs. For a given $H_{x}$, each data point in a cluster represents the (window-averaged) RTD at two successive observation intervals; thus, we generate a cluster of points corresponding to a plot (actually, a "residencetimes return map") of $\Delta_{1}(n+1)$ versus $\Delta_{1}(n)$. Each cluster of points corresponds to one value of the target field $H_{x}$ (in the absence of background noise, each cluster would collapse into a single point for that particular value of $H_{x}$ ); in this experimental sequence (Fig. 21), the point clusters correspond to values of $H_{x}$ that are approximately $2.0 \mathrm{nT}$ apart. One can use a smaller separation of $H_{x}$ values; however, this separation has been chosen for the purposes of elucidation (with smaller separation, the clusters tend to merge). The density function of each cluster is near Gaussian, with a mean value corresponding to the averaged RTD over all the discrete points and a standard deviation that can be computed from the observations. The locus of the mean values is the straight line. When one plots these mean RTD values as a function of $H_{x}$ (not shown), the slope of this line [the responsivity, i.e., the denominator of (37)] is $229.83 s T^{-1}$. In the figure, the standard deviations of the point clusters are (from left to right) $0.057315,0.054994,0.065573$, and $0.04463 \mu \mathrm{s}$, corresponding to resolutions [calculated from (37)] of 250, 240, 286, and $195 \mathrm{pT}$, respectively, resulting in a mean resolution of $242 \mathrm{pT}$ for this particular sensor. The resolution is approximately constant (the deviations arise from experimental uncertainties and fluctuations) in this regime of low target signal.

It is important to realize that, as $H_{x}$ increases, the target signal becomes more easily "resolved." However, the analytic description of the response breaks down when $H_{x}$ becomes comparable to (or exceeds) the energy barrier height of a single-element (isolated) potential function; in this regime, the resolution becomes $H_{x}$ dependent. One expects, at least in the linear response regime $\left(H_{x} x_{0} \ll \Delta U\right.$, where $\Delta U$ is the energy barrier height of a single isolated element), that the distribution

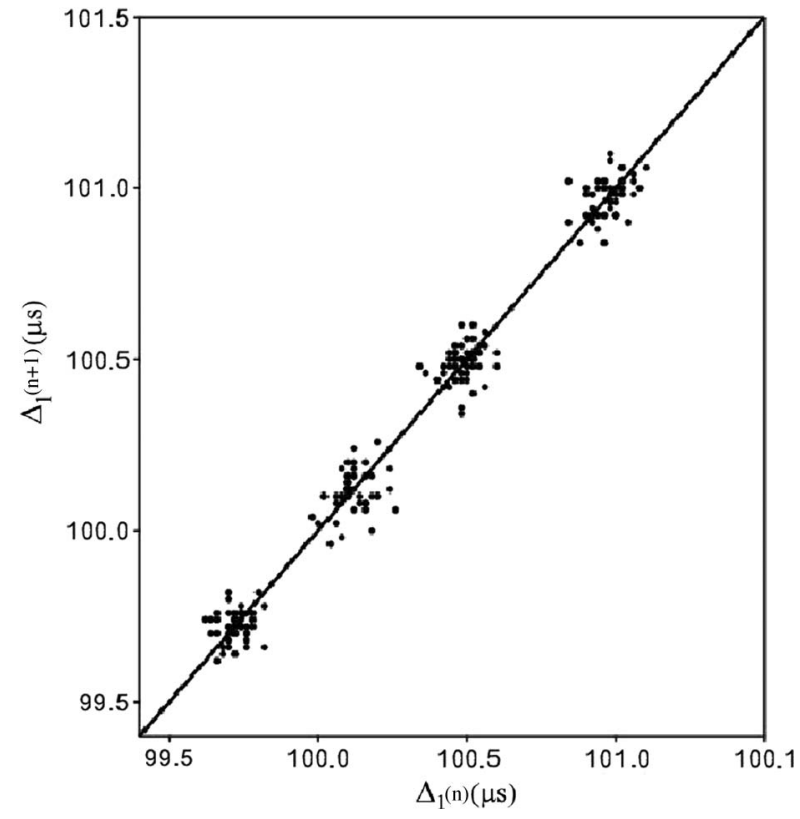

Fig. 21. Return map of the (experimentally obtained) RTDs; each cluster corresponds to a different $H_{x}$, and the straight line is the locus of the means. Each individual point in a cluster corresponds to an average of 15-20 values of the RTD taken in an observation time window (updated every $0.1 \mathrm{~s}$ ). The standard deviation of each cluster is a function of $H_{x}$, while the mean value is proportional to $H_{x}$ so that the responsivity [denominator of (37)] is an $H_{x}$-independent number (see text). At low signal values, the resolution is approximately constant (see text). The parameters are $N=3$ cores and $G=-0.8$. $G$ is an experimentally realized (via the ratio of two resistors in the coupling circuit) gain parameter corresponding to the coupling coefficient $\lambda$ in the theoretical description (figure reproduced with permission from [26]).

of the discrete points corresponding to a given $H_{x}$ will be near Gaussian; this is, in fact, observed in our experiments. The numerator in (37) is also computed, directly from the data, for each value of $H_{x}$. It is important to note that the quantity in (37) incorporates the effects of the noise on the sensor performance in a simple manner that can be easily implemented in practice; we can, in fact, use this quantity to make comparisons between different sensor versions.

\section{Coupled Ferroelectric CAPACitors: An EFS}

It is well known [5] that ferroelectric materials exhibit hysteresis in their polarization versus applied E-field response. This suggests that one might be able to exploit this behavior to realize an EFS with dynamics qualitatively similar to the (single- and coupled-core) fluxgate magnetometer.

EFSs find utility in a number of applications, e.g., geophysical exploration, oceanography, and biomedical imaging/ mapping. The EFSs, used in these applications, usually rely on a computation of (changes in) the potential difference between two electrodes attached to a conducting medium (e.g., the sea bottom for oceanographic experiments); the sensitivity improves with increased baseline, but this also makes the sensors somewhat cumbersome and expensive. Nonetheless, they remain an important imaging tool largely because the terrestrial E-field fluctuations/pulsations are on a far smaller scale than their magnetic counterparts. 


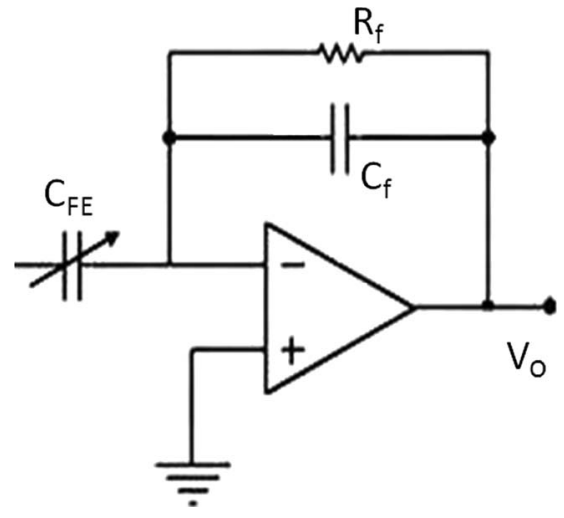

Fig. 22. Simple realization of the "Sawyer-Tower" circuit.

The synergetic exploitation of ferroelectric materials and micromachining technologies affords a route to quantifying charge density amplification in a nonlinear active medium (the ferroelectric material); in turn, this leads to a novel sensing strategy based on coupling nonlinear elemental cells. Essentially, the elementary cell consists of a micromachined capacitor whose core is a ferroelectric material, polarized via an imposed bias $\mathrm{E}$ field. The polarization status is altered when the target $\mathrm{E}$ field (taken to be dc) is superimposed on the (known) bias field. Furthermore, a suitably fabricated external receptor allows amplification of the target field to the sensing element [77].

The nonlinear ferroelectric devices considered in this paper can be modeled by the following differential equation in the polarization $P_{F E}$ :

$$
\tau_{e} \dot{P}_{F E}=a P_{F E}-b P_{F E}^{3}+c E_{x}+E_{e} \sin \omega t .
$$

Again, the over dot denotes the time derivative, and $a, b$, and $\tau_{e}$ denote the material-dependent system parameters governing its bistable behavior; $c$ is a coefficient that characterizes the "strength" of the coupling between the external E fields and the dielectric sample. It is important to note that (46) is, in fact, derived [77], [78] from a mean-field description of the system; we do not reproduce this here, however. The potential energy for the given material is therefore expressed as

$U\left(P_{F E}, t\right)=-\frac{a}{2} P_{F E}^{2}+\frac{b}{4} P_{F E}^{4}-c E_{x} P_{F E}-E_{e} P_{F E} \sin \omega t$.

The E field in the sample can be seen as the result of the contributions coming from an auxiliary E field (the time-sinusoidal bias field) and an external $\mathrm{E}$ field to be detected. The presence of a target signal results in the asymmetry of $U\left(P_{F E}, t\right)$, and detection techniques are aimed at quantifying this asymmetry. Furthermore, in order to characterize the ferroelectric device, a suitable circuit is required; it is based on a charge amplifier and is the "Sawyer-Tower circuit" [6], [79] shown in Fig. 22.

In this circuit, the polarization $P_{F E}$ of the ferroelectric capacitor $C_{F E}$ is proportional to the circuit output voltage $V_{o}$. The definitions of the polarization as a function of E field and the $\mathrm{E}$ flux as a function of capacitance lead to

$$
V_{o}=-\frac{C_{F E}}{C_{f}} V_{e}
$$

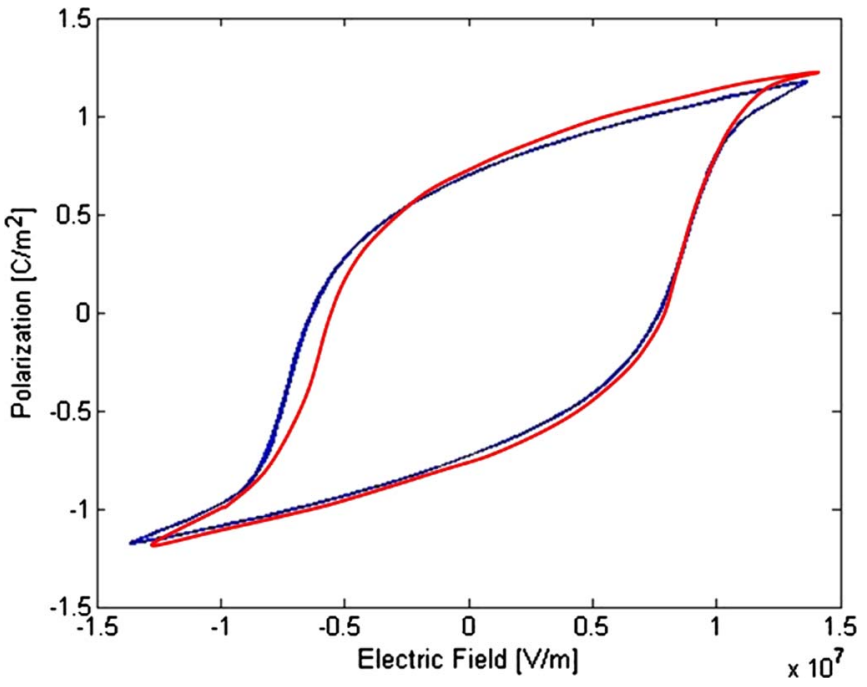

Fig. 23. (Thin red curve) Experimental characterization and (thick black curve) parameter estimation of the hysteresis in the ferroelectric sample; a timeperiodic signal having $10 V_{p p}$ amplitudes and $100-\mathrm{Hz}$ frequency has been used to produce the E field across the capacitor.

that can be rewritten as

$$
V_{o}=-\frac{C_{F E}}{C_{f}} \frac{P_{F E} A_{F E}}{C_{F E}}
$$

and finally lead to

$$
V_{o}=-\frac{A_{F E}}{C_{f}} P_{F E}
$$

Equation (50) expresses the proportionality between the circuit output and the polarization of the ferroelectric capacitor, where $A_{F E}$ is the ferroelectric capacitor area.

One of the relevant technological issues addressed here is the realization of an integrated ferroelectric capacitor in which the bias-electrode geometry must be such to polarize the ferroelectric sample while allowing the target electric field to perturb this dielectric polarization status. If a suitable sinusoidal bias field is imposed on the ferroelectric material through the capacitor bias electrodes, then, ideally, a square wave will occur at the circuit output due to the bistable behavior of the system. The external perturbation to the electrical polarization will be observed via a change in the output signal.

The ferroelectric capacitors investigated in this paper were realized at the Penn State University Laboratories. A common silver electrode was evaporated onto a silicon substrate, the ferroelectric material was deposited over this (bottom) electrode, and, finally, several top electrodes were "spotted" over the top surface of the ferroelectric and bonded to the external connections. The hysteretic behavior of the sample material has been confirmed (in the absence of a target signal) experimentally [77], as shown in Fig. 23, where the Sawyer-Tower output voltage is plotted against the input voltage that is proportional to the bias E field. These experimental observations were used [77] to identify the parameters of the analytical model given in (46); in turn, the analytic model is plotted in the figure with a thicker line.

Operating the EFS as a single-element device, via a reference (i.e., excitation) applied signal $V_{e}$, that produces an excitation 


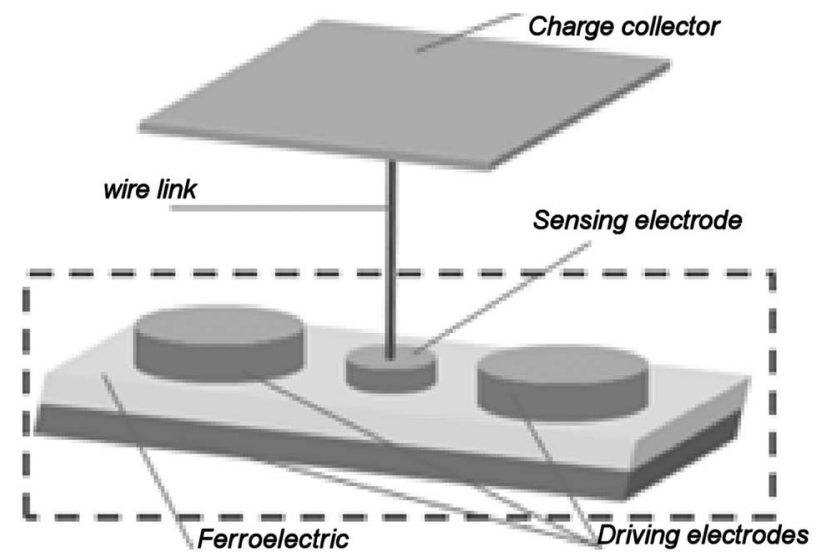

Fig. 24. Schematic view of the charge collection mechanism to enhance sensitivity in ferroelectric EFSs.

E field $E_{e}$ to induce switching between the stable polarization states is however problematic due to the high coercive fields that are typical in ferroelectric materials. This is not an issue for magnetic materials; hence, the SCFG can be readily realized without a very large onboard power consumption. Thus, the idea of coupling an odd number of overdamped bistable elements [each one having dynamics of the form (46)] in a ring, with unidirectional coupling and cyclic boundary conditions, and forcing the system into oscillation is particularly appealing in this case because the "oscillations" do, in fact, correspond to switching over the energy barriers of each elemental potential. In what follows, we report on the effects of inserting a small dc "target" E-field signal into the (coupled) element system, via an appropriate charge collection/amplification mechanism that perturbs the polarization status of the capacitors, as shown in Fig. 24. The charge collection mechanism has been considered in great detail in our recent work [77].

The idea is to use a three-electrode configuration: two for polarizing the ferroelectric layer and a third one to convey the perturbation due to the target field to the sensing region. The latter electrode is wired to a "charge collector" consisting of a conductive plate. The purpose of the "charge collector" is to collect the charges induced by the target $\mathrm{E}$ field; in turn, the collected charge is immediately transferred to the sensing plate, thus perturbing the polarization of the "sensing region." This behavior has been confirmed via a finite-element method analysis [78]; the changes induced in the polarization status of this capacitor manifest themselves in alterations of the output signal from the signal-conditioning circuit.

In analogy to our preceding treatment of coupled ferromagnetic cores, one can explore the nonlinear dynamics of the circuit obtained by unidirectionally coupling an odd number of ferroelectric elements. The circuit implementation of this system is shown in Fig. 25.

The coupled dynamics, for $N=3$ elements, are written in the following form [80]:

$$
\begin{aligned}
& \tau_{e} \dot{P}_{1}=a P_{1}-b P_{1}{ }^{3}+\lambda c\left(V_{O 3}-V_{O 1}\right) \\
& \tau_{e} \dot{P}_{2}=a P_{2}-b P_{2}{ }^{3}+\lambda c\left(V_{O 1}-V_{O 2}\right) \\
& \tau_{e} \dot{P}_{3}=a P_{3}-b P_{3}{ }^{3}+\lambda c\left(V_{O 2}-V_{O 3}\right)
\end{aligned}
$$

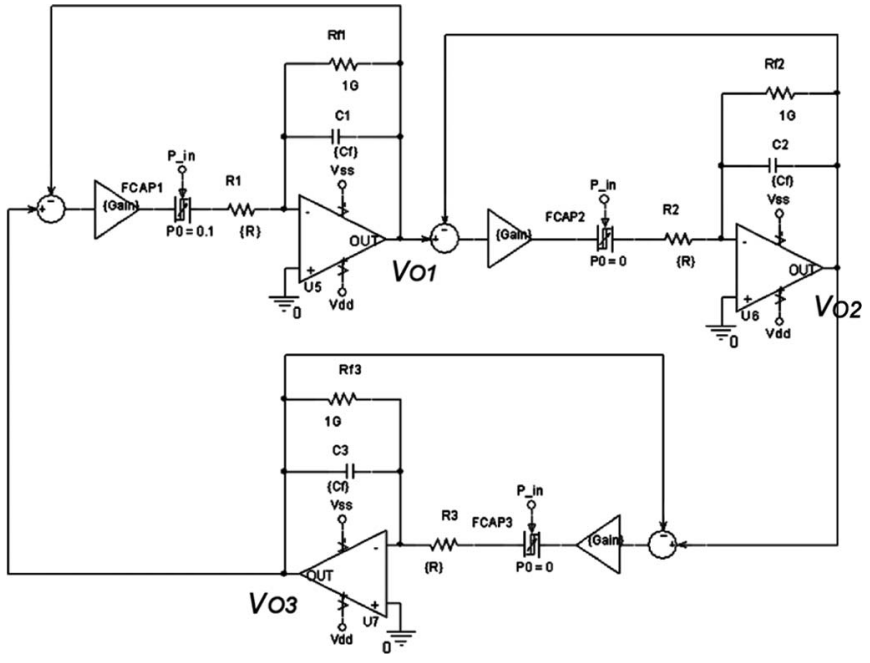

Fig. 25. Coupled ferroelectric capacitor circuit implemented with analog electronics.

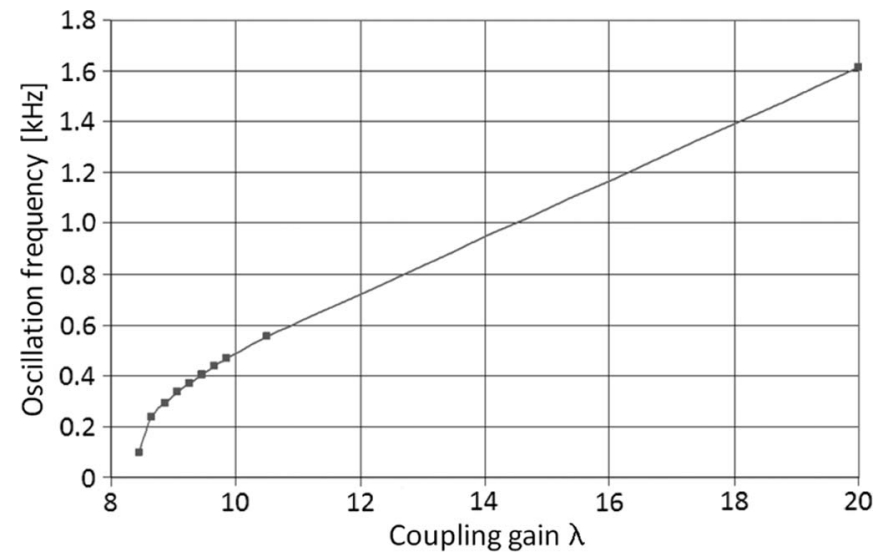

Fig. 26. Coupled ferroelectric capacitor system oscillation frequency versus the coupled gain $\lambda$.

with $\lambda$ being the coupling coefficient, and $a, b$, and $c$ being the potential function parameters that, together with the time constant $\tau_{e}$, can be determined as part of the material characterization process [81].

Simple calculations [80] yield the critical coupling $\lambda_{c}$

$$
\lambda_{c}=\frac{a}{2}+\frac{5}{3} \sqrt{\frac{b}{2 a}} c E_{x}
$$

beyond which the system oscillates. The frequency of the oscillations, as well as the "residence times" of each element in the up and down stable polarization steady states, can be theoretically computed [80] and are found to be in good agreement with experiments and simulations.

The experiments carried out with this coupled circuit yield the expected oscillations in good agreement with theoretical predictions [78]. In Fig. 26, the oscillation frequency is shown as a function of the coupling gain. The existence of a limiting minimum gain value (proportional to $\lambda_{c}$ ), below which the system does not oscillate, is also evident in this figure.

Some other preliminary experimental characterizations have been performed by placing the measurement system between two large parallel conducting plates, used to generate a known 

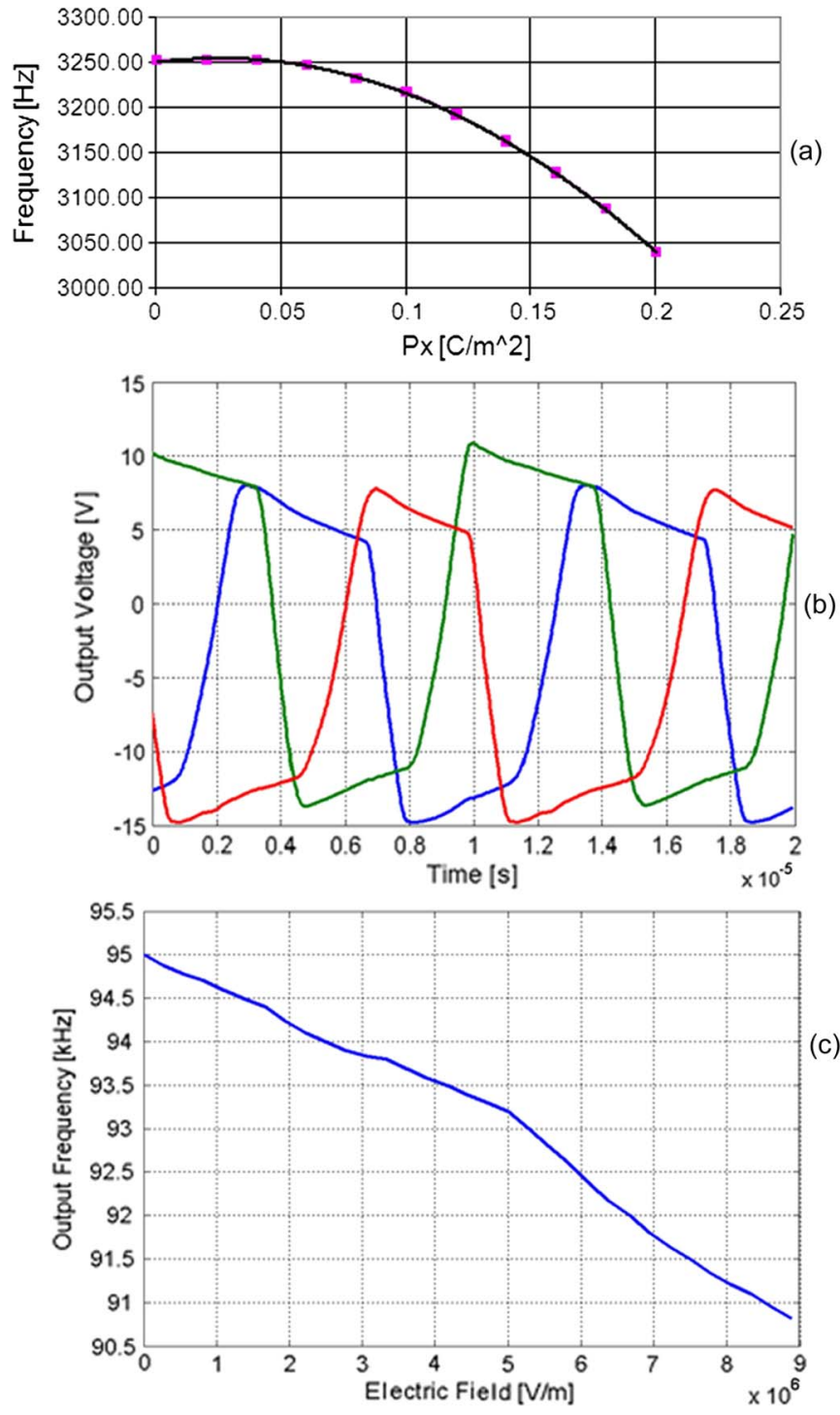

Fig. 27. (a) Simulated results for the output voltage frequency as a function of the charge accumulated on the capacitor plate. (b) Experimental observations of the output voltages; the $2 \pi / 3$ phase shift among the output voltages can be observed. (c) Experimental observations of the changes in the output voltage frequency as a function of the external target $\mathrm{E}$ field. The frequency behavior in (a) and (c) is qualitatively similar to the behavior of the analogous quantity in the CCFG (see, e.g., Fig. 17) and has been discussed rigorously in [80].

target $\mathrm{E}$ field. The experimentally observed change in the position of the main peak in the power spectrum of the output signal is shown in Fig. 27(c), and a good qualitative agreement is obtained with the simulation results shown in Fig. 27(a). The three output voltage waveforms are shown in Fig. 27(b).

\section{Discussion And Conclusion}

In this paper, we have presented an alternative to quantifying the output of a nonlinear dynamic system via the PSD. The residence-times-based technique is relatively simple to implement in practical scenarios; all that are required are for the detection/processing electronics to keep track of the threshold crossing events and maintain a running average, i.e., the arithmetic mean, of the residence times in each stable state. Then, the mean RTD $\langle\Delta T\rangle$ provides a measure of the unknown target signal that created the asymmetry and, therefore, a nonzero $\langle\Delta T\rangle$. While the target signal in this paper is taken to be $\mathrm{dc}$, it is clear that a modification of the residence-times-based readout scheme could be effected for more complex signals; the CCFG lends itself particularly well to this scenario [82]. The choice of the bias signal waveform is important to the issue of the overall resolution defined roughly as the ability to discriminate the means of the residence-times densities in the presence of a small asymmetrizing target signal. In this context, it has been demonstrated [83] that the triangular bias signal yields better performance due to its (local) linearity at the points where the signal intersects one of the thresholds. The bias signal amplitude does not need to be extremely large [13]; this point has been addressed in some detail with the pertinent observation that a bias signal amplitude slightly larger than the deterministic switching threshold (roughly, the energy barrier height) suffices, except for special operating conditions (e.g., a large noise floor).

Clearly, in such a situation, it would be preferable to adjust the system parameters [e.g., the constant $c$ in the potential energy function (2)] so that the energy barrier is lowered when weak target signals are to be detected in a noise floor. Absent such a control, however, adjusting the bias amplitude effectively lowers (or raises) the energy barrier. With a large background noise floor, the density functions tend to merge, leading to inaccuracies in the computed RTD, unless a large number $N$ of observations can be made. Increasing the bias amplitude $\hat{H}_{e}$ enables one to better resolve the density functions, even as it leads to a greater power requirement. Hence, one must also consider the tradeoff between resolution, sensitivity, and power when designing a sensor aimed at a particular class of target signals. Noise effects become more important as the bias signal amplitude approaches the threshold; the RTD is no longer symmetric (about its mean value), it develops tails, and its mean and mode separate. In [83], we employed a stochastic perturbation theory approach to determine the upper bound on the achievable accuracy, via a family of estimation procedures that can be simply implemented and are asymptotically optimal in the vanishing noise limit. Based on these calculations, the SCFG appears to be the optimal magnetometer for some applications involving low-cost compact magnetometers out of the entire class of pulse position modulated fluxgate magnetometers [8], [27], [28].

For the SCFG, the bias frequency does not figure prominently into the crossing statistics when we work in the nondynamical limit (i.e., the limit wherein the device time constant is the smallest timescale in the dynamics); however, in the general case, the frequency must be carefully selected. In some ferromagnetic cores employed, for instance, in the simple magnetometer used in our experiments, the (non-Gaussian) Barkhausen noise floor depends on the bias frequency through its effect on the slip dynamics of the domain walls; usually, there exists a (material-dependent) optimal frequency at which these effects are negligible [84]. Moreover, for the case of a soft ferromagnetic core, the width of the hysteresis loop, which determines the energy dissipated per cycle, can depend on the frequency and amplitude of the bias signal. Keeping the bias signal amplitude and frequency as low as possible can lead to 
significantly reduced onboard power; in a real device, this can be an important consideration. However, clearly, the tradeoff between onboard power and the observation window update time $T_{\mathrm{ob}}$ (which determines the accuracy of the experimental estimate of the RTD) eventually dictates how the sensor is operated.

The SCFG has been completed and patented. It boasts a field (i.e., unshielded) resolution of $0.5-1.0 \mathrm{nT}$ and currently consumes about $150 \mathrm{~mW}$ of power; the latter number is expected to be significantly reduced in the near future, with the insertion of ultralow-power electronic components that are currently being designed. The sensor is configured to measure the dc component of the ambient magnetic field and null it out (i.e., normalize itself to the ambient field) at start-up; this process, which can be repeated on cue, takes approximately $10 \mathrm{~s}$. The size and weight of the device are predicated by the length (typically $6 \mathrm{~cm}$ ) of the wound core and the power supply. If the onboard power is to be provided by batteries, then the device will be much larger. A current biaxial version of the magnetometer, with an external power source, has $10 \times 10 \times 1 \mathrm{~cm}$ dimensions and weighs about $0.5 \mathrm{~kg}$ (the sensor is "podded" in epoxy for robustness, at the cost of a small increase in weight). The magnetometer employs an (approximately) single-domain microwire core [69], [84] which has been characterized by us [43]. The observation time is $0.1 \mathrm{~s}$. This means that, in processing the output, we average the RTD data using a temporal window of 30 -s width; the window is updated every $0.1 \mathrm{~s}$. The bias frequency is $300 \mathrm{~Hz}$, leading to an acceptable number of data points in the 30-s window, and the bias amplitude is taken to be approximately 1.25 times the energy barrier height.

We have also developed a laboratory version of the CCFG with the AO configuration. This sensor yields a dynamic behavior that faithfully follows all the theoretical predictions. The laboratory (i.e., shielded) resolution of this sensor is $200 \mathrm{pT}$; however, its power consumption is currently quite high. In principle, the resolution can be improved (i.e., the numerical value decreases) by incorporating a larger number of cores; this is readily apparent when we realize that the denominator of (37) scales linearly as $N$ [this is also seen in the theoretical approximation (45)]. However, increasing the number of cores comes at the cost of increased engineering complexity and additional onboard power (for the coupling circuitry). In addition, the effects of increasing $N$ on the magnetic noise floor are still under investigation, although the results shown in Fig. 20 provide grounds for optimism that increasing $N$ will not degrade the response as long as we operate within well-defined regimes of optimal response (e.g., near the onset of oscillations, as suggested in Fig. 20). Here, however, an important caveat emptor must be repeated. When the system is tuned very close to the onset of oscillations, the background noise can have a significant effect, particularly if it is large enough so that its variance approaches the energy barrier height. This is the weak coupling regime wherein the input to each element from the element it is coupled to is deterministic but very small compared to the noise floor. In this regime, an experimental time series of the response displays a noise component that decreases as the coupling becomes larger. Accordingly, while the system may exhibit its best (theoretical) response in the lowfrequency regime, a practical system may need to be operated somewhat farther away from the critical point; this option is not available in the SCFG, where a large noise floor is usually offset by increasing the bias signal amplitude at the cost of a larger onboard power requirement. Clearly, this makes the case for using every available means to a priori lower the system noise floor.

Currently, the CCFG draws greater power than its SCFG counterpart. This is largely due to the coupling circuitry and the need to drive $N$ cores instead of one (in the SCFG). However, this is balanced by a significantly enhanced performance, quantified by the resolution, as well as the experimentally observed lower (compared to the SCFG) noise floor. In this context, it must be noted (also in connection with the CCFG) that, even though a bias signal is not explicitly inserted into the coupled dynamics, there is still a power supply on board the sensor to power electronic components (op-amps, etc). Thus, the emergent oscillations do not violate any fundamental laws. Future plans call for the realization of these circuits in lowpower CMOS, which should lead to a reduction in the power budget for both sensors. In both sensors (CCFG and SCFG), following the nulling procedure, the sensor detects very small static magnetic fields (which can be far below the terrestrial magnetic field) in motion.

We also point out that, in recent laboratory realizations of the CCFG, the parameter mismatch issues have been reduced to a minimum. The cores are near identical (cut from the same sample of single-domain magnetic wire), and the coupling circuitry can also be set up so that the coupling coefficients $\lambda$ are almost the same throughout the arrangement. Hence, while there are likely to be lingering mismatches in the parameter values, they can be substantially minimized. Of greater concern then are the sources of noise arising from the readout electronics, the magnetic noise in the cores themselves, and fluctuations that are superimposed on the target signal. The effects of fluctuations have been addressed [76] in a very preliminary way, using a somewhat simplistic model of fluctuations in only one parameter (the target signal) and assuming moreover that the fluctuations occur on the timescale of the observation time. This yields a theoretical expression for the resolution that faithfully reproduces qualitatively the behavior observed in experiments. We also point to our earlier work [75], [85] in which we described the effects of the fluctuations without the aforementioned timescale restriction and also described some rich noisemediated spatiotemporal behavior in large coupled rings. This leaves us with another potential source of noise that can easily arise in practice, namely, temperature fluctuations that affect the nonlinearity parameter $c$, particularly when the same device is operated in greatly different environments. The parameter $c$ is proportional to the ratio $T_{c} / T, T_{c}$ being the Curie temperature. Temperature fluctuations will therefore introduce complicated state-dependent noise terms in the coupled dynamics. In turn, the parameters $\lambda_{c}$, as well as the positions of the fixed points of the core potential functions (for zero coupling), will also fluctuate.

The EFS follows qualitatively similar physics as the magnetometer; however, the structural difference between the 
potential energy functions (2) and (47) should be noted; clearly, the underlying physics determines the structure of the potential energy in each case. Unlike the magnetometer, however, it can be impractical to operate a single-element version of the EFS because of the very high coercivity associated with ferroelectric materials; this would necessitate a bias signal of very large amplitude and, hence, a significantly larger (compared to the SCFG) power supply. Accordingly, pending the development of a ferroelectric material with lower coercivity, it seems more natural to operate a coupled-element version of the EFS, as presented in Section IV. A detailed laboratory characterization of the single-element EFS, together with the parallel plate mechanism used for charge collection, has been given in [77]. In this work, a reference signal of $10 \mathrm{~V}$ at $100 \mathrm{~Hz}$ yielded very good sensitivity (as characterized by the slope of the polarization versus applied E-field characteristic) with a noise floor of $0.4(\mathrm{~V} / \mathrm{m}) / \mathrm{Hz}^{1 / 2}$.

While the coupled-element EFS has already been realized in the laboratory, we call attention to a very recent [86] bistable microelectronic circuit for sensing extremely low electric field. This circuit comprises three coupled elements; however, the structure of the nonlinearity is very different from (51), with both the coupling and the nonlinearity realized via hyperbolic tangent functions that are reminiscent of the "soft" potential (2) that characterizes the magnetic system. The coupling is again taken to be unidirectional, and the dynamics are qualitatively similar to (38) and (51). This microelectronic circuit realization is an obvious extension (and exploitation) of the coupled system dynamics that underpin the coupled-core magnetometer and the coupled-element EFS presented in the current work. The circuit yields effectively an extremely sensitive detector of very low $\mathrm{E}$ current that can be realized on a chip; the entire laboratory layout, including the readout electronics, currently occupies a $4 \mathrm{~cm} \times 4 \mathrm{~cm}$ circuit board. Thus, the bistable microelectronic circuit provides a striking demonstration of the utility of nonlinear dynamical systems which, when properly understood, could provide new and exciting sensing paradigms.

It is important to realize that our coupling scheme is quite general; it can readily be applied to a vast array of dynamical systems which follow the basic "particle-in-potential" paradigm of the form $\dot{x}=-\nabla_{x} U(x)$, with $U$ being any bi- or multistable potential and $x$ being the appropriate state variable. The ability to control the oscillation frequency (our system can be made to oscillate at frequencies ranging from a few hertz to several kilohertz) dramatically broadens the range of applications that can benefit from this scheme. In a recent work [82], we have demonstrated that the CCFG can be used to detect time-periodic target magnetic fields by exploiting the very rich nonlinear mixing behavior from the interaction of the target frequency with the internal oscillation frequency.

As a final note, it is worth reiterating that the idea of threshold crossing events leading to a quantification of external signals is deeply rooted in the computational neuroscience repertoire wherein one analyzes the response of a single neuron, or even a small network, to a stimulus by examining the statistics of the point process generated by successive threshold crossings or "firings." Thus, our proposed mode of operation actually leads to an implementation of these sensors as "neural-like" devices.

\section{REFERENCES}

[1] W. Geyger, Nonlinear Magnetic Control Devices. New York: McGrawHill, 1964.

[2] F. Forster, "A method for the measurement of DC field differences and its application to nondestructive testing," Nondestruct. Test, vol. 13, p. 31, 1955.

[3] A. Barone and G. Paterno, Physics and Applications of the Josephson Effect. New York: Wiley, 1982.

[4] J. Clarke, "Squids: Theory and practice," in The New Superconducting Electronics, H. Weinstock and R. Ralston, Eds. Amsterdam, The Netherlands: Kluwer, 1993.

[5] I. Bunget and M. Prohescu, Physics of Solid Dielectrics. New York: Elsevier, 1984.

[6] D. Damjanovic, P. Muralt, and N. Setter, "Ferroelectric sensors," IEEE Sensors J., vol. 1, no. 3, pp. 191-206, Oct. 2001

[7] J. Fraden, Handbook of Modern Sensors. New York: AIP, 1997.

[8] W. Bornhofft and G. Trenkler, Sensors, A Comprehensive Survey, W. Gopel, J. Hesse, and J. Zemel, Eds. New York: VCH, 1989.

[9] M. Inchiosa and A. Bulsara, "DC signal detection via dynamical asymmetry in a nonlinear device," Phys. Rev. E, Stat. Phys. Plasmas Fluids Relat. Interdiscip. Top., vol. 58, no. 1, pp. 115-127, Jul. 1998.

[10] S. Y. Yurish, "Digital sensors design based on universal frequency sensors interfacing IC," Sens. Actuators A, Phys., vol. 132, no. 1, pp. 265-270, Nov. 2006.

[11] S. Y Yurish, F. Reverter, and R. Pallàs-Areny, "Measurement error analysis and uncertainty reduction for period- and time-interval-to-digital converters based on microcontrollers," Meas. Sci. Technol., vol. 16, no. 8, pp. 1660-1666, Aug. 2005.

[12] L. Gammaitoni and A. Bulsara, "Noise-activated nonlinear dynamic sensors," Phys. Rev. Lett., vol. 88, no. 23, p. 230 601, Jun. 2002.

[13] A. Bulsara, C. Seberino, L. Gammaitoni, M. Karlsson, B. Lundqvist, and J. Robinson, "Signal detection via residence times asymmetry in noisy bistable devices," Phys. Rev. E, Stat. Phys. Plasmas Fluids Relat. Interdiscip. Top., vol. 67, no. 1, p. 016120, Jan. 2003.

[14] L. Gammaitoni, F. Marchesoni, E. Menichella-Saetta, and S. Santucci, "Stochastic resonance in bistable systems," Phys. Rev. Lett., vol. 62, no. 4, pp. 349-352, Jan. 1989.

[15] A. R. Bulsara and L. Gammaitoni, "Tuning into noise," Phys. Today, vol. 49, no. 3, pp. 39-47, Mar. 1996.

[16] T. Zhou, F. Moss, and P. Jung, "Escape time distributions of a periodically modulated bistable system with noise," Phys. Rev. A, Gen. Phys., vol. 42, no. 6, pp. 3161-3169, Sep. 1990.

[17] M. Choi, R. Fox, and P. Jung, "Quantifying stochastic resonance in bistable systems: Response vs. residence times distribution functions," Phys. Rev. E, Stat. Phys. Plasmas Fluids Relat. Interdiscip. Top., vol. 57, no. 6, pp. 6335-6344, Jun. 1998.

[18] A. Longtin, A. Bulsara, and F. Moss, "Time interval sequences in bistable systems and the noise-induced transmission of information by sensory neurons," Phys. Rev. Lett., vol. 67, no. 5, pp. 656-659, Jul. 1991.

[19] A. Longtin, A. Bulsara, D. Pierson, and F. Moss, "Bistability and the dynamics of periodically forced sensory neurons," Biol. Cybern., vol. 70, no. 6, pp. 569-578, Apr. 1994.

[20] C. Koch, Biophysics of Computation. New York: Oxford Univ. Press, 1999.

[21] D. Cox, Point Processes. New York: CRC Press, 1980.

[22] A. Nikitin, N. Stocks, and A. Bulsara, "Asymmetric bistable systems subject to periodic and stochastic forcing in the strongly nonlinear regime: Switching time distributions," Phys. Rev. E, Stat. Phys. Plasmas Fluids Relat. Interdiscip. Top., vol. 68, no. 1, p. 016103, Jul. 2003.

[23] A. Nikitin, N. Stocks, and A. Bulsara, "Asymmetric bistable systems subject to periodic and stochastic forcing in the strongly nonlinear regime: Signal-to-noise ratio," Phys. Rev. E, Stat. Phys. Plasmas Fluids Relat. Interdiscip. Top., vol. 76, no. 4, p. 041138, Oct. 2007.

[24] G. Papoulis, Probability, Random Variables, and Stochastic Processes. New York: McGraw-Hill, 1991.

[25] A. Bulsara, V. In, A. Kho, P. Longhini, A. Palacios, W. Rappel, J. Acebron, S. Baglio, and B. Andò, "Emergent oscillations in unidirectionally coupled overdamped bistable systems," Phys. Rev. E, Stat. Phys. Plasmas Fluids Relat. Interdiscip. Top., vol. 70, no. 3, p. 036103, Sep. 2004.

[26] A. Bulsara, V. In, A. Kho, P. Longhini, A. Palacios, G. Anderson, S. Baglio, and B. Andò, "Exploiting nonlinear dynamics in a coupled-core fluxgate magnetometer," Meas. Sci. Technol., vol. 19, no. 7, p. 075203, Jul. 2008 .

[27] P. Ripka, "Review of fluxgate sensors," Sens. Actuators A, Phys., vol. 33, no. 3, pp. 129-141, Jun. 1992. 
[28] P. Ripka, Magnetic Sensors and Magnetometers. Boston, MA: Artech House, 2001.

[29] F. Kaluza, A. Gruger, and H. Gruger, "New and future applications of fluxgate sensors," Sens. Actuators A, Phys., vol. 106, no. 1-3, pp. 48-51, Sep. 2003.

[30] A. Tipek, P. Ripka, T. O’Donnell, and J. Kubik, "PCB sensor technology used in fluxgate sensor construction," Sens. Actuators A, Phys., vol. 115, no. 2/3, pp. 286-292, Sep. 2004.

[31] P. Kejik, L. Chiesi, B. Janossy, and R. S. Popovic, "A new compact 2D planar fluxgate sensor with amorphous metal core," Sens. Actuators A, Phys., vol. 81, no. 1-3, pp. 180-183, Apr. 2000.

[32] L. Chiesi, P. Kejik, B. Janossy, and R. S. Popovic, "CMOS planar 2D micro-fluxgate sensor," Sens. Actuators A, Phys., vol. 82, no. 1-3, pp. 174-180, May 2000

[33] R. Koch, J. Deak, and G. Grinstein, "Fundamental limits to magnetic field sensitivity of fluxgate magnetic field sensors," Appl. Phys. Lett., vol. 75, no. 24, pp. 3862-3864, Dec. 1999.

[34] D. Gordon and R. Brown, "Recent advances in fluxgate magnetometry," IEEE Trans. Magn., vol. MAG-8, no. 1, pp. 76-82, Mar. 1972.

[35] Å. Forslund, S. Belyayev, N. Ivchenko, G. Olsson, T. Edberg, and A. Marusenkov, "Miniaturized digital fluxgate magnetometer for small spacecraft applications," Meas. Sci. Technol., vol. 19, no. 1, p. 015202, Jan. 2008.

[36] W. Magnes, D. Pierce, A. Valavanoglou, W. Baumjohann, J. Means, C. T. Russell, K. Schwingenschuh, and G. Graber, "A sigma-delta fluxgate magnetometer for space applications," Meas. Sci. Technol., vol. 14, no. 7, pp. 1003-1012, Jul. 2003.

[37] S. C. Tang, M. C. Duffy, and P. Ripka, "Application of fluxgate excitation circuit with saturable inductor to magnetic sensing," Sens. Actuators A, Phys., vol. 123/124, pp. 430-437, Sep. 2005.

[38] Bartington fluxgate magnetometers. [Online]. Available: www. bartington.com

[39] W. Telford, L. Geldart, and R. Sheriff, Applied Geophysics. Cambridge, U.K.: Cambridge Univ. Press, 1990.

[40] B. Andò, S. Baglio, A. Bulsara, and V. Sacco, "Residence times difference fluxgate magnetometers," IEEE Sensors J., vol. 5, no. 5, pp. 895-904, Oct. 2005.

[41] G. Vertesy, J. Szollosy, L. K. Varga, and A. Lovas, "High sensitivity magnetic field sensors using amorphous alloy," Electron. Horiz., vol. 102, p. 53, 1992.

[42] S. Strycker and A. Wulkan, "A pulse position type fluxgate magnetometer," AIEE Trans., vol. 80, pp. 253-257, 1961.

[43] B. Andò, S. Baglio, A. Bulsara, and C. Trigona, "Design and characterization of a microwire fluxgate magnetometer," Sens. Actuators A, Phys., vol. 151, no. 2, pp. 145-153, Apr. 2009

[44] G. Bertotti, Hysteresis in Magnetism. San Diego, CA: Academic, 1998.

[45] H. E. Stanley, Introduction to Phase Transitions and Critical Phenomena. New York: Oxford Univ. Press, 1971.

[46] T. Tome and M. de Oliveira, "Dynamic phase transition in the kinetic Ising model under a time-dependent oscillating field," Phys. Rev. A, Gen. Phys., vol. 41, no. 8, pp. 4251-4254, Apr. 1990.

[47] H. Fujisaka, H. Tutu, and P. Rikvold, "Dynamic phase transition in a time-dependent Ginzburg-Landau model in an oscillating field," Phys. Rev. E, Stat. Phys. Plasmas Fluids Relat. Interdiscip. Top., vol. 63, no. 3, p. 036109, Mar. 2001.

[48] S. Sides, P. Rikvold, and M. Novotny, "Kinetic Ising model in an oscillating field: Finite-size scaling at the dynamic phase transition," Phys. Rev. Lett., vol. 81, no. 4, pp. 834-837, Jul. 1998.

[49] S. Sides, P. Rikvold, and M. Novotny, "Kinetic Ising model in an oscillating field: Avrami theory for the hysteretic response and finite-size scaling for the dynamic phase transition," Phys. Rev. E, Stat. Phys. Plasmas Fluids Relat. Interdiscip. Top., vol. 59, no. 3, pp. 2710-2729, Mar. 1999.

[50] Z. Neda, "Stochastic resonance in Ising systems," Phys. Rev. E, Stat. Phys. Plasmas Fluids Relat. Interdiscip. Top., vol. 51, no. 6, pp. 5315-5317, Jun. 1995.

[51] W. F. Brown, "Thermal fluctuations of a single-domain particle," Phys. Rev., vol. 130, no. 5, pp. 1677-1686, Jun. 1963.

[52] L. Landau and E. Lifshitz, Electrodyanmics of Continuous Media. New York: Pergamon, 1960.

[53] A. Perez-Madrid and J. M. Rubi, "Stochastic resonance in a system of ferromagnetic particles," Phys. Rev. E, Stat. Phys. Plasmas Fluids Relat. Interdiscip. Top., vol. 51, no. 5, pp. 4159-4164, May 1995.

[54] P. Jung, G. Gray, and R. Roy, "Scaling laws for dynamic hysteresis," Phys. Rev. Lett., vol. 15, p. 65, 1990.

[55] B. Andò, S. Baglio, A. Bulsara, and V. Sacco, "Residence times difference fluxgate," Measurement, vol. 38, no. 2, pp. 89-112, Sep. 2005.
[56] J. Millman, Microelectronics. New York: McGraw-Hill, 1983.

[57] B. McNamara and K. Wiesenfeld, "Theory of stochastic resonance," Phys. Rev. A, Gen. Phys., vol. 39, no. 9, pp. 4854-4869, May 1989.

[58] F. Primdahl, "The fluxgate mechanism part I: The gating curves of parallel and orthogonal fluxgates," IEEE Trans. Magn., vol. MAG-6, no. 2, pp. 376-383, Jun. 1970.

[59] C. Gardiner, Handbook of Stochastic Methods. Berlin, Germany: Springer-Verlag, 1985

[60] L. Gammaitoni, F. Marchesoni, and S. Santucci, "Stochastic resonance as a bonafide resonance," Phys. Rev. Lett., vol. 74, no. 7, pp. 1052-1055, Feb. 1995.

[61] A. Bulsara, M. Karlsson, J. Robinson, and L. Gammaitoni, "Exploiting nonlinear dynamics in a fluxgate magnetometer," in Proc. 3rd Int. Conf. MARELEC, Stockholm, Sweden, 2001.

[62] L. Gammaitoni, A. Dari, and L. Bosi, "Nonlinear sensors: An approach to the residence times detection strategy," Phys. Rev. E, Stat. Phys. Plasmas Fluids Relat. Interdiscip. Top., vol. 81, no. 1, p. 011115, Jan. 2010.

[63] L. Gammaitoni and A. Dari, "Noisy nonlinear detectors," in Applications of Nonlinear Dynamics: Model and Design of Complex Systems. New York: Springer-Verlag, 2009, pp. 225-235.

[64] B. Ando, S. Baglio, A. R. Bulsara, and S. La Malfa, "ROC analysis for RTD fluxgate magnetometers," in Proc. IEEE I2MTC, Austin, TX, 2010, pp. $163-166$.

[65] J. Roj, J. Jakubiec, and P. Makowski, "Error model application in neural reconstruction of nonlinear sensor input signal," IEEE Trans. Instrum. Meas., vol. 58, no. 3, pp. 649-656, Mar. 2009.

[66] Evaluation of Measurement Data-Guide to the Expression of Uncertainty in Measurement, ISO/IEC Guide 98-3, 2008.

[67] N. Bleistein and R. Handelsman, Asymptotic Expansions of Integrals. New York: Dover, 1986.

[68] [Online]. Available: www.metglas.com

[69] H. Chiriac, J. Yamasaki, T. A. Ovari, and M. Takajo, "Magnetic domain structure in amorphous glass-covered wires with positive magnetostriction," IEEE Trans. Magn., vol. 35, no. 5, pp. 3901-3903, Sep. 1999.

[70] B. Andò, S. Baglio, A. Bulsara, S. La Malfa, and C. Trigona, "Experimental investigations on the spatial resolution in RTD-fluxgates," in Proc. IEEE I2MTC, 2009, pp. 1542-1545.

[71] B. Andò, A. Bulsara, S. Baglio, V. Caruso, and V. Sacco, "Investigating the optimal geometry to minimize the demagnetizing effect in RTDfluxgate," in Proc. IEEE IMTC, 2006, pp. 2175-2178.

[72] B. Andò, S. Baglio, S. La Malfa, A. Bulsara, and C. Trigona, "Perming effect in residence times difference fluxgate magnetometers," in Proc. AISEM, 2009, pp. 257-260.

[73] M. Hernandez, V. In, P. Longhini, A. Palacios, A. Bulsara, and A. Kho, "Coupling-induced oscillations in nonhomogenous overdamped bistable systems," Phys. Lett. A, vol. 372, no. 24, pp. 4381-4387, Jun. 2008.

[74] A. Palacios, J. Aven, V. In, P. Longhini, A. Kho, J. Neff, and A. Bulsara, "Coupled core fluxgate magnetometer: Novel configuration scheme and the effects of a noise-contaminated external signal," Phys. Lett. A, vol. 367, no. 1/2, pp. 25-34, Jul. 2007.

[75] A. Bulsara, J. Lindner, V. In, A. Kho, S. Baglio, V. Sacco, B. Andò, P. Longhini, A. Palacios, and W. J. Rappel, "Coupling-induced cooperative behavior in dynamic ferromagnetic cores in the presence of a noise floor," Phys. Lett. A, vol. 353, no. 1, pp. 4-10, Apr. 2006.

[76] A. Bulsara, V. In, A. Kho, G. Anderson, P. Longhini, J. Neff, S. Baglio, B. Andò, and A. Palacios, "Time domain quantification of the performance of a nonlinear dynamic device in the presence of a noise floor," Eur. Phys. J. B, vol. 69, no. 1, pp. 109-118, May 2009.

[77] S. Baglio, V. Marletta, B. Andò, and A. R. Bulsara, "A ferroelectric capacitor based approach to quasi static electric field sensing," IEEE Trans. Instrum. Meas., vol. 59, no. 3, pp. 641-652, Mar. 2010.

[78] S. Baglio, A. R. Bulsara, N. Savalli, B. Andò, and V. In, "Electric field detectors in a coupled ring configuration: Preliminary results," in Proc. SPIE Conf., Adelaide, Australia, 2006, p. 641702.

[79] S. Sivasubramanian, A. Widom, and Y. Srivastava, "Equivalent circuit and simulations for the Landau-Khalatnikov model of ferroelectric hysteresis," IEEE Trans. Ultrason., Ferroelectr., Freq. Control, vol. 50, no. 8 , pp. 950-957, Aug. 2003.

[80] V. In, A. Palacios, A. Bulsara, P. Longhini, A. Kho, J. Neff, S. Baglio, and B. Andò, "Complex behavior in driven unidirectionally coupled overdamped duffing elements," Phys. Rev. E, Stat. Phys. Plasmas Fluids Relat. Interdiscip. Top., vol. 73, no. 6, p. 066121, Jun. 2006.

[81] P. Giannone, S. Graziani, and B. Andò, "Dynamic characterisation and model identification in ferroelectric thin films," Int. J. Model., Identif. Control, vol. 3, no. 4, pp. 404-412, Sep. 2008 
[82] V. In, A. Bulsara, A. Palacios, P. Longhini, and A. Kho, "Complex dynamics in unidirectionally coupled overdamped bistable systems subject to a time-periodic external signal," Phys. Rev. E, Stat. Phys. Plasmas Fluids Relat. Interdiscip. Top., vol. 72, no. 4, p. 045104, Oct. 2005.

[83] M. Karlsson, J. Robison, A. Bulsara, and L. Gammaitoni, "The optimal achievable accuracy of the advanced dynamic magnetometer," in Proc. 3rd Int. Conf. MARELEC, Stockholm, Sweden, 2001.

[84] R. Koch and J. Rozen, "Low noise fluxgate magnetic field sensors using ring and rod core geometries," Appl. Phys. Lett., vol. 78, no. 13, pp. 18971899, Mar. 2001.

[85] J. Lindner and A. Bulsara, "One-way coupling enables noise-mediated spatiotemporal patterns in media of otherwise quiescent multistable elements," Phys. Rev. E, Stat. Phys. Plasmas Fluids Relat. Interdiscip. Top., vol. 74, no. 2, p. 020105(R), Aug. 2006.

[86] V. In, P. Longhini, N. Liu, A. Kho, J. Neff, A. Palacios, and A. Bulsara, "A bistable microelectronic circuit for sensing extremely low electric field," J. Appl. Phys., vol. 107, no. 1, p. 014506, Jan. 2010.

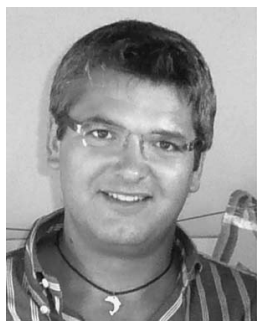

Salvatore Baglio (S'93-M'95-SM'03) received the "Laurea" and Ph.D. degrees from the Università degli Studi di Catania, Catania, Italy, in 1990 and 1994, respectively.

Since 1996, he has been with the Dipartimento di Ingegneria Elettrica Elettronica e dei Sistemi, Università degli Studi di Catania, where he is currently an Associate Professor. He teaches courses in "measurement theory," "electronic instrumentations," and "integrated microsensors." $\mathrm{He}$ is the coauthor of more than 250 scientific publications, including books, chapters in books, and papers in international journals and proceedings of international conferences. He is the holder of several U.S. and European patents. His research interests are mainly focused on micro- and nanotechnologies, micro- and nanosensors, hysteretic materials for sensors, and nonlinear dynamics for transducers.

Dr. Baglio has served as an Associate Editor for the IEEE TRANSACTIONS ON CIRCUITS AND SYSTEMS and as a Distinguished Lecturer for the IEEE Circuits and Systems Society. He is currently an Associate Editor of the IEEE TRANSACTIONS ON INSTRUMENTATION AND MEASUREMENT and the Chair of the Italian IEEE Instrumentation and Measurement Italy Chapter.

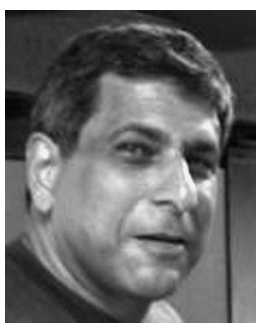

Adi R. Bulsara received the Ph.D. degree in physics from the University of Texas, Austin, in 1978.

$\mathrm{He}$ is currently a Senior Researcher with the Space and Naval Warfare Systems Center Pacific, San Diego, CA, where he heads a group that specializes in applications of nonlinear dynamics. He is the author of more than 150 articles in physics. His research interest includes the physics of noisy nonlinear dynamic systems, with a preference for applications.

Dr. Bulsara is a Fellow of the American Physical

Society.

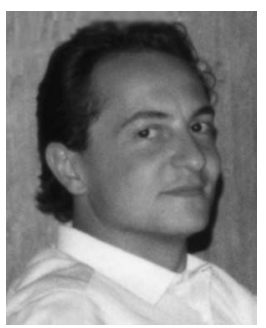

Bruno Andò received the M.S. degree in electronic engineering and the Ph.D. degree in electrical engineering from the Università degli Studi di Catania, Catania, Italy, in 1994 and 1999, respectively.

From 1999 to 2001, he was a Researcher with the Electrical and Electronic Measurement Group, Dipartimento di Ingegneria Elettrica, Elettronica e dei Sistemi, Università degli Studi di Catania. In 2002, he became an Assistant Professor with the same staff. During his activity, he has coauthored several scientific papers, presented in international conferences and published in international journals and books. His main research interests are sensor design and optimization, advanced multisensors architecture for ambient assisted living, sensor networks, characterization of new materials for sensors, nonlinear techniques for signal processing with particular interest in stochastic resonance and dithering applications, and distributed measurement systems.

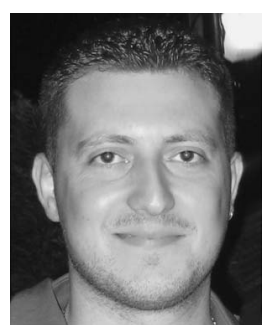

Salvatore La Malfa was born in Milazzo, Italy, on April 16, 1984. He received the B.S. degree (with full honors) in computer science engineering and the M.S. degree (with full honors) in automation engineering and control of complex system from the Università degli Studi di Catania, Catania, Italy, in 2006 and 2008, respectively, where he has been working toward the Ph.D. degree in electronic and automation engineering, and control of complex systems (XXIV cycle) since 2008. His B.S. thesis discussed the metrological characterization of RTD fluxgate magnetometers, while his M.S. thesis discussed the fluxgate magnetometers for biomedical applications.

Since 2008, he has been a Contract Research Engineer with the Dipartimento di Ingegneria Elettrica Elettronica e dei Sistemi, Università degli Studi di Catania. His research interests include fluxgate magnetometers, assistive technologies for visually impaired people, and analog and digital electronic circuit designs.

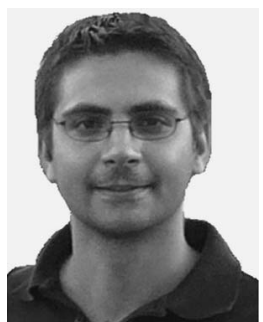

Vincenzo Marletta received the M.S. degree from the Università degli Studi di Catania, Catania, Italy, in 2007, where he is currently working toward the $\mathrm{Ph} . \mathrm{D}$. degree in the Dipartimento di Ingegneria Elettrica, Elettronica e dei Sistemi.

His research interests include sensor design and characterization, particularly the aids for visually impaired people; characterization of new materials for sensors; soft-computing methodologies for instrumentation and measuring systems; smart sensors; and exploitation of nonlinear dynamics in sensors, microsensors, and microsystems in standard and dedicated technologies.

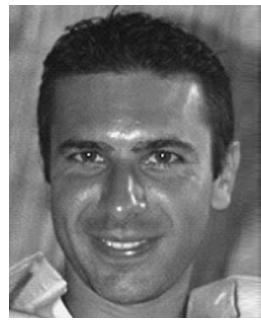

Carlo Trigona was born in Siracusa, Italy, in 1981. $\mathrm{He}$ received the M.S. degree in automation engineering and control of complex system and the Ph.D. degree in electronic, automation, and control of complex system from the Università degli Studi di Catania, Catania, Italy, in 2006 and 2009 respectively.

He is the coauthor of more than 40 scientific publications, which include chapters in books and papers in international journals and proceedings of international conferences. His research interests include microsystems and microsensors, energy harvesting, fluxgate magnetometers, and nonlinear systems.

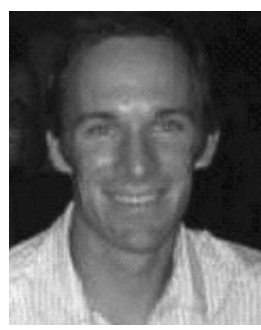

Patrick Longhini received the Ph.D. degree in computation sciences from San Diego State University, San Diego, CA, in collaboration with Claremont Graduate University, Claremont, CA, in 2005.

$\mathrm{He}$ currently holds a position at the Space and Naval Warfare Systems Center Pacific, U.S. Navy, San Diego. He has copublished numerous theoretical and experimental papers in journals, such as Physical Review Letters, Physical Review E, and Proceedings of the IEEE. His main research interest is in the numerical analysis. field of nonlinear dynamics in both theoretical and

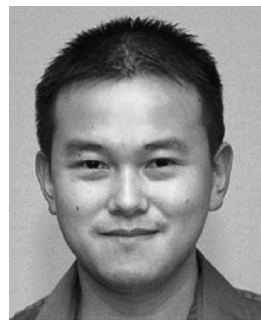

Andy Kho received the B.S. degree in electrical engineering from the Georgia Institute of Technology, Atlanta, in 2001.

$\mathrm{He}$ is currently with the Space and Naval Warfare Systems Center Pacific, U.S. Navy, San Diego, $\mathrm{CA}$, doing experiments in the field of nonlinear dynamics. He specializes in analog electronics and printed circuit board design. He is currently working on coupled nonlinear systems with special emphasis on electric and magnetic field sensors and RF communications. 


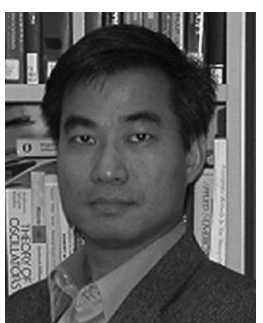

Visarath In received the Ph.D. degree in physics from the Georgia Institute of Technology, Atlanta, in 1996.

He is currently with the Space and Naval Warfare Systems Center Pacific, U.S. Navy, San Diego, CA. He works in the field of nonlinear dynamics and has in-depth experience in the areas relating to mechanical, electronic, and nonlinear sensor systems. He is currently working on coupled nonlinear systems with special emphasis on fluxgate magnetic sensors, electric-field sensors, coupled nonlinear gyroscopes for navigation, signal generation and multiple frequency generation systems, and nonlinear channelizer system for RF communication.

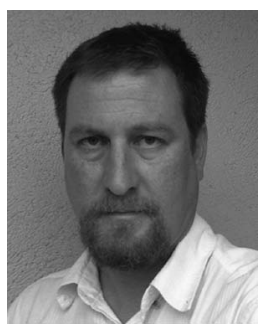

Joseph D. Neff received the B.A. degree from the College of Wooster, Wooster, OH, in 1993 and the $\mathrm{Ph} . \mathrm{D}$. degree in physics from the Georgia Institute of Technology, Atlanta, in 2000. While at Georgia Institute of Technology, he studied the control of cardiac chaos, control of flameout in pulse combustor engines and reconfigurable electronics.

He is currently with the Space and Naval Warfare Systems Center Pacific, U.S. Navy, San Diego, CA, where he is a member of the Advanced Dynamics Research Group. His current areas of interest include low-power electronics, circuits operating in extreme environments, and noisedriven computation.

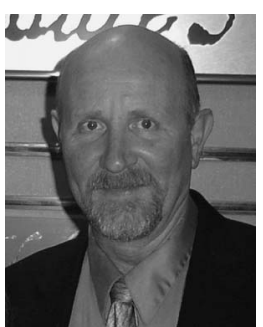

Gregory W. Anderson received the B.S. degree in mechanical engineering from the University of Washington, Seattle, in 1976 and the M.S. degree in mechanical engineering from San Diego State University, San Diego, CA, in 1981.

He started his professional career in 1976 at the Naval Undersea Center, San Diego. He is currently with the Space and Naval Warfare Systems Center Pacific, U.S. Navy, San Diego, where he has served as the Head of the Applied Technology and Research Branch since 1998 and is currently a Project Engineer for the prototype development of the advanced dynamics magnetometer. He was the Team Leader for the first SPAWAR San Diego Innovation Cell (in 2005 ) and has worked on a wide range of engineering projects, from submarine drag reduction to remote autonomous environmental sensor systems. He was a Guest Lecturer at the University of California, San Diego (in 1996-2000).

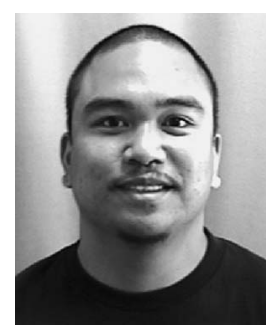

Chris C. Obra received the B.S. degree in electrical engineering from San Diego State University, San Diego, CA.

$\mathrm{He}$ is currently an Electronics Engineer with the Applied Research Branch, Space and Naval Warfare Systems Center Pacific, U.S. Navy, San Diego.

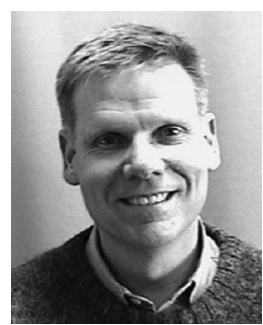

Brian K. Meadows received the B.S. degree in physics from the University of California, Irvine, in 1993 and the Ph.D. degree in physics from the Georgia Institute of Technology, Georgia, in 1998.

$\mathrm{He}$ is currently a Senior Scientist with the Space and Naval Warfare Systems Center Pacific, U.S. Navy, San Diego, CA.

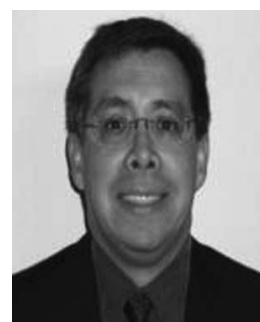

Antonio Palacios received the B.S. degree in computer engineering from La Salle University, Mexico, in 1986 and the M.S. degree in computational science, the M.S. degree in computer science, and the $\mathrm{Ph} . \mathrm{D}$. degree in applied mathematics from Arizona State University, Tempe, in 1990, 1994, and 1995 , respectively.

He is currently a Professor of applied mathematics with San Diego State University, San Diego, CA. His research interests are in modeling and analyzing complex nonlinear systems, particularly bifurcation theory in systems with symmetry. 